\title{
Motivic amplitudes and cluster coordinates
}

\author{
J.K. Golden, ${ }^{a}$ A.B. Goncharov, ${ }^{b}$ M. Spradlin, ${ }^{a, c}$ C. Vergu ${ }^{d}$ and A. Volovich ${ }^{a, c}$ \\ ${ }^{a}$ Department of Physics, Brown University, \\ Box 1843, Providence, RI 02912-1843, U.S.A. \\ ${ }^{b}$ Department of Mathematics, Yale University, \\ PO Box 208283, New Haven, CT 06520-8283, U.S.A. \\ ${ }^{c}$ Theory Division, Physics Department, CERN, \\ 1211 Geneva 23, Switzerland \\ ${ }^{d}$ ETH Zürich, Institut für Theoretische Physik, \\ Wolfgang Pauli Strasse 27, 8093 Zürich, Switzerland \\ E-mail: John_Golden@brown.edu, sasha.goncharov@gmail.com, \\ Marcus_Spradlin@brown.edu, c.vergu@gmail.com, \\ Anastasia_Volovich@brown.edu
}

ABSTRACT: In this paper we study motivic amplitudes - objects which contain all of the essential mathematical content of scattering amplitudes in planar SYM theory in a completely canonical way, free from the ambiguities inherent in any attempt to choose particular functional representatives. We find that the cluster structure on the kinematic configuration space $\operatorname{Conf}_{n}\left(\mathbb{P}^{3}\right)$ underlies the structure of motivic amplitudes. Specifically, we compute explicitly the coproduct of the two-loop seven-particle MHV motivic amplitude $\mathcal{A}_{7,2}^{\mathcal{M}}$ and find that like the previously known six-particle amplitude, it depends only on certain preferred coordinates known in the mathematics literature as cluster $\mathcal{X}$-coordinates on $\operatorname{Conf}_{n}\left(\mathbb{P}^{3}\right)$. We also find intriguing relations between motivic amplitudes and the geometry of generalized associahedrons, to which cluster coordinates have a natural combinatoric connection. For example, the obstruction to $\mathcal{A}_{7,2}^{\mathcal{M}}$ being expressible in terms of classical polylogarithms is most naturally represented by certain quadrilateral faces of the appropriate associahedron. We also find and prove the first known functional equation for the trilogarithm in which all 40 arguments are cluster $\mathcal{X}$-coordinates of a single algebra. In this respect it is similar to Abel's 5-term dilogarithm identity.

KEYwords: Supersymmetric gauge theory, Scattering Amplitudes

ARXIV EPRINT: 1305.1617 


\section{Contents}

1 Introduction 1

2 The kinematic configuration space $\operatorname{Conf}_{n}\left(\mathbb{P}^{3}\right) \quad 4$

2.1 Momentum twistors 4

2.2 Bracket notation 5

2.3 Configurations and Grassmannians $\quad 6$

$\begin{array}{ll}2.4 \text { The euclidean region } & 7\end{array}$

3 Review of the two-loop $n=6 \mathrm{MHV}$ amplitude $\quad 8$

4 Polylogarithms and motivic Lie algebras $\quad 10$

$\begin{array}{ll}\text { 4.1 The motivic avatars of (generalized) polylogarithms } & 10\end{array}$

$\begin{array}{ll}4.2 & \text { Higher Bloch groups } \\ & 12\end{array}$

$\begin{array}{lll}4.3 & \text { Symbols } & 16\end{array}$

$\begin{array}{ll}4.4 \text { Motivic scattering amplitudes } & 16\end{array}$

5 The coproducts of two-loop MHV motivic amplitudes 17

$\begin{array}{ll}5.1 \text { The } \Lambda^{2} \mathrm{~B}_{2} \text { component for } n=7 & 17\end{array}$

$\begin{array}{lll}5.2 & \text { The } \mathrm{B}_{3} \otimes \mathbb{C}^{*} \text { component for } n=7 & 18\end{array}$

6 Cluster coordinates and cluster algebras $\quad 19$

$\begin{array}{ll}6.1 \text { Introduction and definitions } & 20\end{array}$

$\begin{array}{ll}\text { 6.2 Cluster Poisson varieties } & 22\end{array}$

6.3 Grassmannian cluster algebras and cluster Poisson spaces $\operatorname{Conf}_{n}\left(\mathbb{P}^{k-1}\right) \quad 23$

$\begin{array}{ll}6.4 & \text { Generalized Stasheff polytopes }\end{array}$

6.5 Poisson bracket and generalized Stasheff polytopes 27

$\begin{array}{lll}6.6 & \text { Parity invariance } & 28\end{array}$

6.7 Cluster algebras and the positive Grassmannian 31

7 Cluster coordinates and motivic analysis for $n=6,7 \quad 31$

$\begin{array}{lll}7.1 & \text { Clusters and coordinates for } \operatorname{Gr}(2,6) & 31\end{array}$

7.2 The generalized Stasheff polytope for $\operatorname{Gr}(2,6) \quad 34$

$\begin{array}{lll}7.3 & \text { Cluster coordinates for } \operatorname{Gr}(3,7) & 37\end{array}$

7.4 Structure of the motivic two-loop $n=7 \mathrm{MHV}$ amplitude 38

8 Conclusion $\quad 39$

A Parity conjugation on $\operatorname{Conf}_{n}\left(\mathbb{P}^{k-1}\right) \quad 41$

A.1 Positive configurations 41

A.2 Parity conjugation 41

A.3 Parity conjugation on configurations of vectors 42 


\section{Introduction}

In the past several years a great amount of attention has been focused on the problem of understanding the hidden mathematical structure of scattering amplitudes (for reviews see [1-6]), particularly (but certainly not exclusively) in supersymmetric theories such as $\mathcal{N}=4$ Yang-Mills (SYM) theory [7, 8]. As amplitudeologists, our mathematical interest in planar SYM theory stems from imagining it as a vast and mysterious encyclopedia, recovered from some long-lost desert cave, filled with functions having remarkable properties and interrelationships. This encyclopedia has many volumes, but beyond the most introductory sections, we can only make out bits and scraps of text here and there.

It is hardly our ambition to greatly ameliorate this situation. Rather, our goal in this work is to describe some general mathematical properties of and techniques for analyzing amplitudes - to provide a kind of archaeologist's toolkit. In particular, one overarching aim of our work is to point out that SYM theory is an ideal setting in which to study motivic amplitudes, as proposed a decade ago in [9] (see in particular section 7). Why motivic amplitudes? It remains an important outstanding problem in physics to determine explicit effective constructions for general amplitudes. However the abundance of functional identities amongst generalized polylogarithms apparently precludes the existence of any particular preferred or canonical functional representation or 'formula' for general multi-loop amplitudes (the only exception is reviewed in section 3). Our goal is rather to investigate, following [9], their motivic avatars - motivic amplitudes — which are mathematically more sophisticated, but at the same time much more structured and canonical objects. In particular they are elements of a Hopf algebra. This Hopf algebra is the algebra of functions on the so-called motivic Galois group. The group structure of the latter is encoded in the coproduct of the Hopf algebra. So by studying the coproduct of motivic amplitudes - a structure totally invisible if we remain on the level of functions - we uncover their hidden motivic Galois symmetries. One cannot resist to think that these new symmetries will eventually play an essential role in physics.

A similar upgrading, from multi-zeta values to motivic multi-zeta values, has recently played a crucial role in unlocking the structure of tree-level superstring amplitudes in the $\alpha^{\prime}$ expansion [10-13]. In SYM theory we expect the motivic approach to be even more powerful since the amplitudes we deal with are not merely numbers but highly nontrivial functions on the $3(n-5)$-dimensional kinematic configuration space $\operatorname{Conf}_{n}\left(\mathbb{P}^{3}\right)$, the space of collections of $n$ points in the projective space $\mathbb{P}^{3}$, considered modulo the action of the projective linear group $\mathrm{PGL}_{4}$.

The one-sentence slogan of our paper is that we find that the cluster structure of the space $\operatorname{Conf}_{n}\left(\mathbb{P}^{3}\right)$ underlies the structure of amplitudes in $S Y M$ theory. The technical aspects 
of our work which support this conclusion can be divided into two parts, which one can think of very roughly as kinematics and dynamics.

We can phrase the 'kinematic' question we are interested in roughly as: which variables do motivic amplitudes in SYM theory depend on? Thanks to dual conformal symmetry [14$21]$ it is known that appropriately defined $n$-particle SYM scattering amplitudes depend on $3(n-5)$ algebraically independent dual conformal cross-ratios. However, all experience to date indicates that the functional dependence of amplitudes on these variables always takes very special forms. For example, in the case of the two-loop MHV amplitude for $n=6$ (reviewed in section 3), which can be completely expressed in terms of the classical polylogarithm functions $\mathrm{Li}_{m}$ [22], only very particular algebraic functions of the three independent cross-ratios appear as arguments of the $\mathrm{Li}_{m}$ 's. It is natural to wonder why these particular arguments appear, and not others, and to ask about the arguments appearing in more general amplitudes (including $n>6$, higher loops, and non-MHV).

There is a more specific purely mathematical reason to concentrate on the study of the motivic two-loop MHV amplitudes. These amplitudes are polylogarithm-like functions of weight (also known as transcendentality) four. Any such function of weight one on a space $X$ is necessarily of the form $\log F(x)$, the logarithm of a rational function on $X$. Next, any weight two function can be expressed as a sum of $\mathrm{Li}_{2}$ 's and products of two logarithms of rational functions. Similarly, any weight three function is a linear combination of $\mathrm{Li}_{3}$ 's and products of lower weight polylogarithms of rational functions. So the question 'which variables these functions depend on' is well-defined, up to the functional equations satisfied by $\mathrm{Li}_{2}$ and $\mathrm{Li}_{3}-$ a beautiful subject on its own. However this is no longer true for functions of weight four [23]. There is an invariant associated to any weight four function, with values in an Abelian group $\Lambda^{2} \mathrm{~B}_{2}$, reviewed in section 4, which is the obstruction for the function to be expressible as a sum of products of classical polylogarithms $\mathrm{Li}_{m}$. This makes the above question ill-defined. However, upgrading weight four functions to their motivic avatars one sees that their coproducts are expressible via classical motivic polylogarithms of weights $\leq 3$, and so the question makes sense again. Finally, the coproduct preserves all information about motivic amplitudes but an additive constant. So to understand two-loop amplitudes we want first to find explicit formulas for the coproduct of their motivic avatars. On the other hand, the two-loop amplitudes are natural weight four functions, and so one can hope that their detailed analysis might shed a new light on the fundamental unsolved mathematical problems which we face starting at weight four. Therefore, although the level of precision one can reach studying the two-loop motivic MHV amplitudes is unsustainable for higher loops, one can hope to discover general features by looking at the simplest case.

In this paper we propose that the variables which appear in the study of the MHV amplitudes in SYM theory belong to a class known in the mathematics literature as cluster $\mathcal{X}$-coordinates $[24]$ on the configuration space $\operatorname{Conf}_{n}\left(\mathbb{P}^{3}\right)$.

Cluster $\mathcal{X}$-coordinates in general describe Poisson spaces which are in duality with cluster algebras, originally discovered in $[25,26]$. In particular, the space $\operatorname{Conf}_{n}\left(\mathbb{P}^{3}\right)$ is equipped with a natural Poisson structure, invariant under cyclic shift of the points. This Poisson structure looks especially simple in the cluster $\mathcal{X}$-coordinates: the logarithms of the latter provide collections of canonical Darboux coordinates. It seems remarkable that 
the arguments of the amplitudes have such special Poisson properties, although at the moment we do not know how to fully exploit this connection.

An immediate consequence of the cluster structure of the space $\operatorname{Conf}_{n}\left(\mathbb{P}^{3}\right)$ is that its real part $\operatorname{Conf}_{n}\left(\mathbb{R P}^{3}\right)$ contains the domain $\operatorname{Conf}_{n}^{+}\left(\mathbb{R P}^{3}\right)$ of positive configurations of $n$ points in $\mathbb{R P}^{3}$. This positive domain is evidently a part of the Euclidean domain in $\operatorname{Conf}_{n}\left(\mathbb{C P}^{3}\right)$, the domain where amplitudes are singularity-free.

The configuration space $\operatorname{Conf}_{n}\left(\mathbb{P}^{3}\right)$ can be realized as a quotient of the Grassmannian $\operatorname{Gr}(4, n)$ by the action of the group $\left(\mathbb{C}^{*}\right)^{n-1}$. This Grassmannian, describing the external kinematic data of an amplitude, may look unrelated to those which star in [27-32] and involve also 'internal' data related to loop integration variables. However the cluster structure and in particular the positivity play a key role in the Grassmannian approach to amplitudes [32], and we have no doubt that a tight connection between these objects will soon emerge.

Once one accepts the important role played by cluster coordinates as the kinematic variables which, in particular, the coproduct of the two-loop motivic MHV amplitudes are 'allowed' to depend on, it is natural to ask the 'dynamic' question: what exactly is the dependence on these coordinates? For example, what explains the precise linear combination of $\mathrm{Li}_{4}$ functions appearing in the two-loop MHV amplitude for $n=6$ ? There is a vast and rich mathematical literature on cluster algebras, which are naturally connected [33] to beautiful combinatorial structures known as cluster complexes and, more specifically, generalized associahedrons (or generalized Stasheff polytopes) [34, 35]. We defer most of the dynamic question to subsequent work but report here the first example of a connection between these mathematical structures and motivic amplitudes: we find that the 'distance' between a two-loop amplitude and the classical $\mathrm{Li}_{4}$ functions, expressed in the $\Lambda^{2} \mathrm{~B}_{2}$-obstruction, is naturally formulated in terms of certain two-dimensional quadrilateral faces of the associahedron for $\operatorname{Conf}_{n}\left(\mathbb{P}^{3}\right)$. Equivalently, the pairs of functions entering the Stasheff polytope $\Lambda^{2} \mathrm{~B}_{2}$-obstruction for the two-loop MHV amplitudes Poisson commute.

The outline of this paper is as follows. In section 2 we briefly review various notations for configurations of points in $\mathbb{P}^{k-1}$ and the appearance of the $3(n-5)$-dimensional space $\operatorname{Conf}_{n}\left(\mathbb{P}^{3}\right)$ as the space on which $n$-particle scattering amplitudes in SYM theory are defined. We also review the relationship with the Grassmannian $\operatorname{Gr}(4, n)$. In section 3 we call attention to the very special arguments appearing inside the $\mathrm{Li}_{4}$ functions in the two-loop MHV amplitude for $n=6$. Section 4 reviews the mathematics necessary for the calculus of motivic amplitudes. We present our result for the coproduct of the two-loop $n=7$ MHV motivic amplitude in section 5 (results for all higher $n$ will be given in a subsequent publication). In section 6 we turn to cluster algebras related to $\operatorname{Gr}(4, n)$, the construction of cluster coordinates, and the Stasheff polytope and cluster $\mathcal{X}$-coordinates for $\operatorname{Conf}_{n}\left(\mathbb{P}^{3}\right)$. Finally section 7 exhibits these concepts for $n=6,7$ in detail and contains some analysis of the structure of the two-loop $n=7 \mathrm{MHV}$ motivic amplitude and its relation to the Stasheff polytope. While the $n=6$ case is well-known in the mathematical literature, the geometry of the cluster $\mathcal{X}$-coordinates in the $n=7$ case is more intricate. In appendix $\mathrm{A}$ we discuss parity conjugation, and show how to calculate its action on the cluster $\mathcal{X}$-coordinates. In appendix B we discuss and prove the 40-term functional equation for the trilogarithm, which plays a role in section 5 . 


\section{The kinematic configuration space $\operatorname{Conf}_{n}\left(\mathbb{P}^{3}\right)$}

Having argued that scattering amplitudes are a collection of very interesting functions, we begin by addressing a seemingly simple-minded question: what variables do these functions depend on? Despite initial appearances this is far from a trivial question, and somewhat surprisingly a completely satisfactory understanding has only emerged rather recently.

\subsection{Momentum twistors}

The basic problem is essentially this: a scattering amplitude of $n$ massless particles depends on $n$ four-momenta $p_{i}$ (which we can take to be complex), but these are constrained variables. First of all each one should be light-like, $p_{i}^{2}=0$ with respect to the Minkowski metric for all $i$, and secondly energy-momentum conservation requires that $p_{1}+\cdots+p_{n}=0$. These constraints carve out a non-trivial subvariety of $\mathbb{C}^{4 n}$. It is desirable to employ a set of unconstrained variables which parametrize precisely this subvariety. A solution to the problem is provided by momentum twistors [36], whose construction we now review.

In the planar limit of SYM theory we have an additional, and crucial, piece of structure: the $n$ particles come together with a specified cyclic ordering. This arises because each particle lives in the adjoint representation of a gauge group and each amplitude is multiplied by some invariant constructed from the gauge group generators of the participating particles. In the planar limit we take the gauge group to be $\mathrm{U}(N)$ with $N \rightarrow \infty$, in which case only amplitudes multiplying a single trace $\operatorname{Tr}\left[T^{a_{1}} \cdots T^{a_{n}}\right]$ of gauge group generators are nonvanishing.

Armed with a specified cyclic ordering of the particles, the conservation constraint is solved trivially by parameterizing each $p_{i}=x_{i-1}-x_{i}$ in terms of $n$ dual coordinates $x_{i}$. The $x_{i}$ specify the vertices, in $\mathbb{C}^{4}$, of an $n$-sided polygon whose edges are the vectors $p_{i}$, each of which is null. A very special feature of SYM theory in the planar limit is that all amplitudes are invariant under conformal transformations in this dual space-time [14-21]

It is often useful, especially when one is interested in discussing aspects of conformal symmetry, to compactify the space-time. For example, in Euclidean signature, a single point at infinity has to be included in order for conformal inversion to make sense. It is also convenient to complexify space-time. Different real sections of this complexified space correspond to different signatures of the space-time metric. The complexified compactification $\widetilde{M}_{4}$ of four-dimensional space-time is the Grassmannian manifold $\operatorname{Gr}(2,4)$ of two-dimensional vector spaces in a four-dimensional complex vector space $V_{4}$; in other words, there is a one-to-one correspondence between points in $\widetilde{M}_{4}$ and two-dimensional vector subspaces in $V_{4}$. We can projectivize this picture to say that the correspondence is between points in complexified compactified space-time and lines in $\mathbb{P}^{3}$.

In the Grassmannian picture two points are light-like separated if their corresponding 2-planes intersect. So after projectivization, a pair of light-like separated points in $\widetilde{M}_{4}$ corresponds to a pair of intersecting lines in $\mathbb{P}^{3}$. Conformal transformations in space-time correspond to $\mathrm{PGL}_{4}$ transformations on $\mathbb{P}^{3}$.

This $\mathbb{P}^{3}$ space is called twistor space in the physics literature. The importance of this space was first noted in the work of Penrose [37, 38] and more recently emphasized 
by Witten [39] in the context of Yang-Mills theory scattering amplitudes. However the twistors we need here are not the ones associated to the space-time in which the scattering takes place, but rather the ones associated to the dual space mentioned above, where the $x_{i}$ live and on which dual conformal symmetry acts. These were called 'momentum twistors' in ref. [36], where they were first introduced. Momentum twistor space has both a chiral supersymmetric version (see ref. [40]) and a non-chiral supersymmetric version (see refs. [41-43]), but we will not make use of these extensions in this paper.

To summarize: a scattering amplitude depends on a cyclically ordered collection of points $x_{i}$ in the complexified momentum space $\mathbb{C}^{4}$, each of which corresponds to a projective line in momentum twistor space. Since each $x_{i}$ is null separated from its neighbors $x_{i-1}$ and $x_{i+1}$, their corresponding lines in momentum twistor space intersect. We denote by $Z_{i} \in \mathbb{P}^{3}$ the intersection of the lines corresponding to the points $x_{i-1}$ and $x_{i}$. Conversely, an ordered sequence of points $Z_{1}, \ldots, Z_{n} \in \mathbb{P}^{3}$ determines a collection of $n$ lines which intersect pairwise and therefore correspond to $n$ light-light separated points $x_{i}$ in the dual Minkowski space.

\subsection{Bracket notation}

The space we have just described - the collection of $n$ ordered points in $\mathbb{P}^{3}$ modulo the action of $\mathrm{PGL}_{4}$ - defines $\operatorname{Conf}_{n}\left(\mathbb{P}^{3}\right)$, read as 'configurations of $n$ points in $\mathbb{P}^{3}$. Scattering amplitudes of $n$ particles in SYM theory are functions on this $3(n-5)$-dimensional kinematic domain. This space can be essentially realized as the quotient $\operatorname{Gr}(4, n) /\left(\mathbb{C}^{*}\right)^{n-1}$ of the Grassmannian by considering the space of $4 \times n$ matrices (being the homogeneous coordinates of the $n$ points in $\mathbb{P}^{3}$ ) modulo the left-action of $\mathrm{PGL}_{4}$ as well as independent rescaling of the $n$ columns. In this presentation the natural dual conformal covariant objects are four-brackets of the form $\langle i j k l\rangle:=\operatorname{det}\left(Z_{i} Z_{j} Z_{k} Z_{l}\right)$, which is just the $\mathbb{C}^{4}$ volume of the parallelepiped built on the vectors $\left(Z_{i}, Z_{j}, Z_{k}, Z_{l}\right)$.

More precisely, emphasizing the structures involved, given a volume form $\omega_{4}$ in a fourdimensional vector space $V_{4}$, we can define the bracket $\left\langle v_{1}, v_{2}, v_{3}, v_{4}\right\rangle:=\omega_{4}\left(v_{1}, v_{2}, v_{3}, v_{4}\right)$.

These four-brackets are key players in the rest of our story, so we list here a few of their important features. The Grassmannian duality $\operatorname{Gr}(k, n)=\operatorname{Gr}(n-k, n)$ means that configurations of $n$ points in $\mathbb{P}^{k}$ are dual to configurations of $n$ points in $\mathbb{P}^{n-k-2}$. Explicitly, at six points the relationship between four-brackets in $\mathbb{P}^{3}$ and two-brackets in $\mathbb{P}^{1}$ given by

$$
\langle i j k l\rangle=\frac{1}{2 !} \epsilon_{i j k l m n}\langle m n\rangle, \quad\langle i j\rangle=\frac{1}{4 !} \epsilon_{i j k l m n}\langle k \operatorname{lm} n\rangle,
$$

while the relationship at seven points between four-brackets in $\mathbb{P}^{3}$ and three-brackets in $\mathbb{P}^{2}$ is clearly

$$
\langle i j k l\rangle=\frac{1}{3 !} \epsilon_{i j k l m n p}\langle m n p\rangle, \quad\langle i j k\rangle=\frac{1}{4 !} \epsilon_{i j k l m n p}\langle l m n p\rangle .
$$

We find it useful to exploit this duality for six and seven points when doing so leads to additional clarity. An invariant treatment of this duality is given below in section 2.3.

More complicated $\mathrm{PGL}_{4}$ covariant objects can be formed naturally by using projective geometry inside four-brackets. Such objects will appear later in section 6 , so we review the 
standard notation for them here. Following the $\cap$ notation introduced in ref. [31] we define the four-brackets with an intersection to be

$$
\langle a b(c d e) \cap(f g h)\rangle \equiv\langle a c d e\rangle\langle b f g h\rangle-\langle b c d e\rangle\langle a f g h\rangle .
$$

This composite four-bracket vanishes when the line $(a b)$ and the intersection of planes $(c d e) \cap(f g h)$ lie in a common hyperplane.

Here is a slightly different way to think about (2.3). Consider a pair of vectors $v_{1}, v_{2}$ in a four-dimensional vector space $V_{4}$, and a pair of covectors $f_{1}, f_{2} \in V_{4}^{*}$. Then we set

$$
\left\langle v_{1}, v_{2} ; f_{1}, f_{2}\right\rangle \equiv f_{1}\left(v_{1}\right) f_{2}\left(v_{2}\right)-f_{1}\left(v_{2}\right) f_{2}\left(v_{1}\right) .
$$

To get (2.3) we just take the covectors $f_{1}(*):=\langle c, d, e, *\rangle$ and $f_{2}(*):=\langle f, g, h, *\rangle$.

If we pick a vector $c$ in the intersection of the two hyperplanes, writing them as $\left(c a_{2} b_{2}\right)$ and $\left(c a_{3} b_{3}\right)$, then we can rewrite it in a slightly different notation, making more symmetries manifest:

$$
\left\langle a_{1} b_{1}\left(c a_{2} b_{2}\right) \cap\left(c a_{3} b_{3}\right)\right\rangle=-\left\langle c \mid a_{1} \times b_{1}, a_{2} \times b_{2}, a_{3} \times b_{3}\right\rangle,
$$

Precisely, consider the three-dimensional quotient $V_{4} /\langle c\rangle$ of the space $V_{4}$ along the subspace generated by the vector $c$. The volume form $\omega_{4}$ in $V_{4}$ induces a volume form $\omega_{4}(c, *, *, *)$ in $V_{4} /\langle c\rangle$, and therefore in the dual space $\left(V_{4} /\langle c\rangle\right)^{*}$. So we can define three-brackets $\langle *, *, *\rangle_{c}$ in $\left(V_{4} /\langle c\rangle\right)^{*}$. The other six vectors in eq. (2.5) project to the quotient. Taking the crossproducts $\times$ of consecutive pairs of these vectors, we get three covectors in $\left(V_{4} /\langle c\rangle\right)^{*}$. Their volume $-\left\langle a_{1} \times b_{1}, a_{2} \times b_{2}, a_{3} \times b_{3}\right\rangle_{c}$ equals the invariant (2.4). So we get formula (2.5).

Notice the expansions, where we use $\epsilon_{\alpha \beta \gamma} \epsilon^{\alpha}(\cdot, a, b)=a_{\beta} b_{\gamma}-a_{\gamma} b_{\beta}$ :

$$
\begin{aligned}
\left\langle a_{1} \times b_{1}, a_{2} \times b_{2}, a_{3} \times b_{3}\right\rangle & =\epsilon_{\alpha \beta \gamma} \epsilon^{\alpha}\left(\cdot, a_{1}, b_{1}\right) \epsilon^{\beta}\left(\cdot, a_{2}, b_{2}\right) \epsilon^{\gamma}\left(\cdot, a_{3}, b_{3}\right) \\
& =\left\langle a_{1} a_{2} b_{2}\right\rangle\left\langle b_{1} a_{3} b_{3}\right\rangle-\left\langle b_{1} a_{2} b_{2}\right\rangle\left\langle a_{1} a_{3} b_{3}\right\rangle \\
& =-\left\langle a_{2} a_{1} b_{1}\right\rangle\left\langle b_{2} a_{3} b_{3}\right\rangle+\left\langle a_{2} a_{3} b_{3}\right\rangle\left\langle b_{2} a_{1} b_{1}\right\rangle \\
& =\left\langle a_{3} a_{1} b_{1}\right\rangle\left\langle b_{3} a_{2} b_{2}\right\rangle-\left\langle a_{3} a_{2} b_{2}\right\rangle\left\langle b_{3} a_{1} b_{1}\right\rangle .
\end{aligned}
$$

\subsection{Configurations and Grassmannians}

Let us formulate now the relationship between the Grassmannians $\operatorname{Gr}(k, n)$ and the configuration spaces more accurately. We start with the notion of configurations.

Let $V_{k}$ be a vector space of dimension $k$. Denote by $\operatorname{Conf}_{n}(k)$ the space of orbits of the group $\mathrm{GL}_{k}$ acting on the space of $n$-tuples of vectors in $V_{k}$. We call it the space of configurations of $n$ vectors in $V_{k}$. It is important to notice that the sets of configurations of vectors in two different vector spaces of the same dimension are canonically isomorphic. Denote by $\operatorname{Conf}_{n}\left(\mathbb{P}^{k-1}\right)$ the space of $\mathrm{PGL}_{k}$-orbits on the space of $n$-tuples of points in $\mathbb{P}^{k-1}$, called configurations of $n$ points in $\mathbb{P}^{k-1}$.

We consider an $n$-dimensional 'particle vector space' $\mathbb{C}^{n}$ with a given basis $\left(e_{1}, \ldots, e_{n}\right)$. Then a generic $k$-dimensional subspace $h$ in $\mathbb{C}^{n}$ determines a configuration of $n$ vectors $\left(f_{1}, \ldots, f_{n}\right)$ in the dual space $h^{*}$ : these are the restrictions to $h$ of the coordinate linear 
functionals in $\mathbb{C}^{n}$ dual to the basis $\left(e_{1}, \ldots, e_{n}\right)$. This way we get a well-defined bijection only for generic $h$, referred to mathematically as a birational isomorphism,

$$
\operatorname{Gr}(k, n) \stackrel{\sim}{\longrightarrow} \operatorname{Conf}_{n}(k) .
$$

The group $\left(\mathbb{C}^{*}\right)^{n}$ acts by rescaling in the directions of the coordinate axes in $\mathbb{C}^{k}$. This action transforms into rescaling of the vectors of the configuration space $\operatorname{Conf}_{n}(k)$. The diagonal subgroup $\mathbb{C}_{\text {diag }}^{*} \subset\left(\mathbb{C}^{*}\right)^{n}$ acts trivially. So the quotient group $\left(\mathbb{C}^{*}\right)^{n-1}=\left(\mathbb{C}^{*}\right)^{n} / \mathbb{C}_{\text {diag }}^{*}$ acts effectively. Passing to the quotients we get a birational isomorphism

$$
\operatorname{Gr}(k, n) /\left(\mathbb{C}^{*}\right)^{n-1} \stackrel{\sim}{\longrightarrow} \operatorname{Conf}_{n}\left(\mathbb{P}^{k-1}\right) .
$$

The dualities

$$
\operatorname{Conf}_{n}(k) \stackrel{=}{\longrightarrow} \operatorname{Conf}_{n}(n-k), \quad \operatorname{Conf}_{n}\left(\mathbb{P}^{k}\right) \stackrel{=}{\longrightarrow} \operatorname{Conf}_{n}\left(\mathbb{P}^{n-k-2}\right)
$$

are best understood via the identification with the Grassmannian (2.10), followed by the obvious isomorphism $\operatorname{Gr}(k, n)=\operatorname{Gr}(n-k, n)$, obtained by taking the orthogonal planes.

\subsection{The euclidean region}

Scattering amplitudes in field theory have a complicated singularity structure, including poles and branch cut singularities. However, there are regions in the kinematic space where such singularities are absent. In particular, amplitudes are expected on physical grounds to be real-valued and singularity-free everywhere in the Euclidean region, reviewed in this section. It was discussed in ref. [44] in connection with MHV amplitudes in SYM theory.

The Euclidean region is defined most directly in the dual space parametrized by the $x_{i}$. We impose that the coordinates of the vectors $x_{i}$ are real and

$$
\left(x_{i}-x_{i+1}\right)^{2}=0, \quad\left(x_{i}-x_{j}\right)^{2}<0, \text { otherwise }
$$

where the distance is computed with a metric of signature $(+,-,-,-)$ or $(+,+,-,-)$. These constraints define the Euclidean region in terms of the $x_{i}$ coordinates.

When transformed to twistor coordinates the first constraint in eq. (2.13) is always satisfied. However, the constraint that the components of the vectors $x_{i}$ should be real is harder to impose. We can think about twistors as being spinor representations of the complexified dual conformal group. This complexified dual conformal group has several real sections: $\mathrm{SU}(4)$ which corresponds to Euclidean signature, $\mathrm{SL}(4, \mathbb{R})$ which corresponds to split signature $(+,+,-,-)$ and $\mathrm{SU}(2,2)$ which corresponds to $(+,-,-,-)$ signature.

In fact, there are two kinds of spinor representations which we call twistors (denoted by $Z$ ) and conjugate twistors (denoted by $W$ ). There is a $Z$ and a $W$ for every particle in a scattering process, which we denote by $Z_{i}, W_{i}$. Under the dual conformal group the $Z$ and $W$ twistors and transform in the opposite way. That is, if $M$ is a dual conformal transformation,

$$
W \rightarrow W^{\prime}=W M^{-1}, \quad Z \rightarrow Z^{\prime}=M Z .
$$

This implies that there is an invariant product $W \cdot Z$. 
Now we can study the reality conditions. We will not discuss the Euclidean signature $(+,+,+,+)$ any further since it does not allow light-like separation. For split signature, the twistors transform under $\mathrm{SL}(4, \mathbb{R})$ so they can be taken to be real and independent. For Lorentzian signature $(+,-,-,-)$ the symmetry group is $\mathrm{SU}(2,2)$. If $M \in \mathrm{SU}(2,2)$, then $M^{\dagger} \mathcal{C} M=\mathcal{C}$, where $\mathcal{C}$ is a $(2,2)$ signature matrix which we will take to be real and symmetric. Then $\mathcal{C} Z$ transforms in the same way as $W^{\dagger}$ so we can consistently impose a reality condition $W^{\dagger}=\mathcal{C} Z$. This implies that $\left(W_{i} \cdot Z_{j}\right)^{*}=W_{j} \cdot Z_{i}$. The light-like conditions imply that $W_{i}=Z_{i-1} \wedge Z_{i} \wedge Z_{i+1}$, so the previous reality condition can be written in terms of the $Z$ twistors alone as $\langle i-1 i i+1 j\rangle^{*}=\langle j-1 j j+1 i\rangle$.

Finally, let us translate the second condition in eq. (2.13) into twistor language. Spacetime distances $\left(x_{i}-x_{j}\right)^{2}$ cannot be expressed in twistor variables without first making an arbitrary choice of 'infinity twistor'. However this choice cancels in conformal ratios, and for these the dictionary between space-time and momentum twistor space then implies that

$$
\frac{\langle i i+1 j j+1\rangle\langle k k+1 l l+1\rangle}{\langle i i+1 l l+1\rangle\langle j j+1 k k+1\rangle}>0,
$$

for all $i, j, k, l$ for which none of the four-brackets vanishes. This condition is certainly guaranteed if $\langle i i+1 j j+1\rangle>0$ for all nonvanishing four-brackets of this type.

We therefore define the Euclidean region in momentum twistor space by the condition that $\langle i i+1 j j+1\rangle>0$. It has two sub-regions

$$
\begin{array}{ll}
(2,2) \text { signature: } & \langle i j k l\rangle \in \mathbb{R}, \\
(3,1) \text { signature: } & \langle i-1 i i+1 j\rangle^{*}=\langle j-1 j j+1 i\rangle .
\end{array}
$$

Note that the $(2,2)$ signature region contains the positive Grassmannian which is wellstudied mathematically. In contrast, the $(3,1)$ region does not seem to have been studied in the mathematical literature.

\section{Review of the two-loop $n=6 \mathrm{MHV}$ amplitude}

In the previous section we reviewed that $n$-particle scattering amplitudes in SYM theory are functions on the $3(n-5)$-dimensional space $\operatorname{Conf}_{n}\left(\mathbb{P}^{3}\right)$. It is further believed [32] that any MHV or next-to-MHV (NMHV) amplitude, at any loop order $L$ in perturbation theory, can be expressed in terms of functions of uniform transcendentality weight $2 L$. A goal of this paper is to make a sharper statement about the mathematical structure of these functions. Specifically: that their structure is described by a certain preferred collection of functions on $\operatorname{Conf}_{n}\left(\mathbb{P}^{3}\right)$ which are known in the mathematics literature as cluster $\mathcal{X}$-coordinates. In this section we provide a simple but illustrative example of this phenomenon.

The simplest nontrivial multi-loop scattering amplitude is the two-loop MHV amplitude for $n=6$ particles. This was originally computed numerically in [44, 45], then analytically in $[46,47]$ in terms of generalized polylogarithm functions, and finally in a vastly simplified form in terms of only the classical $\mathrm{Li}_{m}$ functions in [22]. We present it 
here very mildly reexpressed as

$$
R_{6}^{(2)}=\sum_{i=1}^{3}\left(L_{i}-\frac{1}{2} \operatorname{Li}_{4}\left(-v_{i}\right)\right)-\frac{1}{8}\left(\sum_{i=1}^{3} \operatorname{Li}_{2}\left(-v_{i}\right)\right)^{2}+\frac{1}{24} J^{4}+\frac{\pi^{2}}{12} J^{2}+\frac{\pi^{4}}{72},
$$

in terms of the functions

$$
\begin{aligned}
L_{i} & =\frac{1}{384} P_{i}^{4}+\sum_{m=0}^{3} \frac{(-1)^{m}}{(2 m) ! !} P_{i}^{m}\left(\ell_{4-m}\left(x_{i}^{+}\right)+\ell_{4-m}\left(x_{i}^{-}\right)\right), \\
P_{i} & =2 \operatorname{Li}_{1}\left(-v_{i}\right)-\sum_{j=1}^{3} \operatorname{Li}_{1}\left(-v_{j}\right),
\end{aligned}
$$

and

$$
\begin{aligned}
J & =\sum_{i=1}^{3} \ell_{1}\left(x_{i}^{+}\right)-\ell_{1}\left(x_{i}^{-}\right), \\
\ell_{n}(x) & =\frac{1}{2}\left(\operatorname{Li}_{n}(-x)-(-1)^{n} \operatorname{Li}_{n}(-1 / x)\right) .
\end{aligned}
$$

Our aim in reproducing this formula here is to highlight two rather astonishing facts. The first is that the argument of each $\mathrm{Li}_{n}$ function is the negative of one of the simple cross-ratios

$$
\begin{aligned}
& v_{1}=\frac{\langle 35\rangle\langle 26\rangle}{\langle 23\rangle\langle 56\rangle}, \quad v_{2}=\frac{\langle 13\rangle\langle 46\rangle}{\langle 16\rangle\langle 34\rangle}, \quad v_{3}=\frac{\langle 15\rangle\langle 24\rangle}{\langle 45\rangle\langle 12\rangle}, \\
& x_{1}^{+}=\frac{\langle 14\rangle\langle 23\rangle}{\langle 12\rangle\langle 34\rangle}, \quad x_{2}^{+}=\frac{\langle 25\rangle\langle 16\rangle}{\langle 56\rangle\langle 12\rangle}, \quad x_{3}^{+}=\frac{\langle 36\rangle\langle 45\rangle}{\langle 34\rangle\langle 56\rangle}, \\
& x_{1}^{-}=\frac{\langle 14\rangle\langle 56\rangle}{\langle 45\rangle\langle 16\rangle}, \quad x_{2}^{-}=\frac{\langle 25\rangle\langle 34\rangle}{\langle 23\rangle\langle 45\rangle}, \quad x_{3}^{-}=\frac{\langle 36\rangle\langle 12\rangle}{\langle 16\rangle\langle 23\rangle}
\end{aligned}
$$

(or their inverses). We caution the reader that the $x_{i}^{ \pm}$here are the negative of the $x_{i}^{ \pm}$used in [22], while the $v_{i}$ used here are related to the three $u_{i}$ cross-ratios most commonly seen in the literature by $v_{i}=\left(1-u_{i}\right) / u_{i}$. Of course, these 9 variables are not independent the dimension of $\operatorname{Conf}_{6}\left(\mathbb{P}^{3}\right)$ is only three - so one could choose any three of them in terms of which to express all of the others algebraically. It is striking that the argument of each $\mathrm{Li}_{m}$ function in (3.1) is expressible as one of these simple cross-ratios rather than, as might have been the case, some arbitrary algebraic function of cross-ratios.

The second striking fact about (3.1) is that out of the 45 distinct cross-ratios of the form

$$
r(i, j, k, l)=\frac{\langle i j\rangle\langle k l\rangle}{\langle j k\rangle\langle i l\rangle}
$$

only the 9 shown in (3.4) actually appear. Note that here, as throughout the paper, we shall never count both $x$ and $1 / x$ separately.

The presentation of (3.1) we have given here also highlights another theme which will pervade this paper: positivity. The cross-ratios defined in eq. (3.4) all have the manifest property that they are positive whenever each ordered bracket is positive, i.e. whenever 
$\langle i j\rangle>0 \forall i<j$ (see appendix A.1 for additional details on positive configurations). As this example and others to be discussed below suggest, we expect all MHV amplitudes will have particularly rich structure on the positive subset of the domain $\operatorname{Conf}_{n}\left(\mathbb{P}^{3}\right)$. The formula (3.1) is expressed in terms of the natural polylogarithm function on the domain of positive real-valued $x$ :

$$
\operatorname{Li}_{n}(-x)=\int_{\Delta_{x}} \log \left(1+t_{1}\right) d \log t_{2} \wedge \cdots \wedge d \log t_{n}:=L_{n}(x)
$$

where $\Delta_{x}=\left\{\left(t_{1}, \ldots, t_{n}\right): 0<t_{1}<t_{2}<\cdots<t_{n}<x\right\}$. The proper continuation of eq. (3.1) to the part of the Euclidean region outside the positive domain was discussed in [22].

In the rest of this paper we will work almost exclusively not with amplitudes but with coproducts of motivic amplitudes, reviewed in the next section. For such purposes it is sufficient to highlight in $R_{6}^{(2)}$ only the leading terms

$$
R_{6}^{(2)}=\sum_{i=1}^{3} L_{4}\left(x_{i}^{+}\right)+L_{4}\left(x_{i}^{-}\right)-\frac{1}{2} L_{4}\left(v_{i}\right)+\cdots,
$$

where the dots stand for products of functions of lower weight, which are killed by the coproduct $\delta$ reviewed in the next section.

In a certain sense this example is too simple, as this amplitude is likely unique in SYM theory in being expressible in terms of classical polylogarithm functions $\mathrm{Li}_{m}$ only. We do not aim to write explicit formulas for more general amplitudes as there is apparently no particular preferred or canonical functional form, so the question of what variables the function depends on requires a more precise definition involving the more sophisticated mathematics to which we turn our attention in the next section.

\section{Polylogarithms and motivic Lie algebras}

In this section we review some of the necessary mathematical preliminaries on transcendental functions and explain ways of distilling the essential motivic content of such functions. The precise mathematical definitions of motivic avatars of polylogarithm-like functions is given in [9]. Taking for granted that such avatars exist, our goal is to provide the elements of motivic calculus necessary to describe their basic properties.

\subsection{The motivic avatars of (generalized) polylogarithms}

Let us start with the motivic background. Given any field $F$, there is an as yet hypothetical mathematical object called the motivic Tate Lie coalgebra $\mathcal{L}_{\bullet}(F)$ of this field [48]. It is graded by positive integers, the weights, i.e. one has

$$
\mathcal{L} \bullet(F)=\bigoplus_{n=1}^{\infty} \mathcal{L}_{n}(F)
$$

There is a cobracket $\delta$, which is a weight preserving linear map

$$
\delta: \mathcal{L}_{\bullet}(F) \longrightarrow \Lambda^{2} \mathcal{L}_{\bullet}(F)
$$


It satisfies the property that the following composition is zero:

$$
\mathcal{L}_{\bullet}(F) \stackrel{\delta}{\longrightarrow} \Lambda^{2} \mathcal{L} \bullet(F) \stackrel{\delta \wedge \mathrm{Id}-\mathrm{Id} \wedge \delta}{\longrightarrow} \Lambda^{3} \mathcal{L} \bullet(F) .
$$

The very existence of this object is known only when $F$ is a number field [49].

Denote by $V^{*}$ the dual vector space to a vector space $V$. If each of the weight components $\mathcal{L}_{n}(F)$ were a finite-dimensional vector space, ${ }^{1}$ this would mean that the dual graded vector space, defined as

$$
\mathrm{L} \bullet(F):=\bigoplus_{n=1}^{\infty} \mathrm{L}_{-n}(F), \quad \mathrm{L}_{-n}(F):=\left(\mathcal{L}_{n}(F)\right)^{*},
$$

is a graded Lie algebra, with the bracket dual to the map $\delta$. Then the condition (4.3) follows from the Jacobi identity.

Consider the universal enveloping algebra $\mathrm{U}_{\bullet}(F)$ of the Lie algebra $\mathrm{L}_{\bullet}(F)$. It is graded by non-positive integers. By definition, $\mathrm{U}_{0}(F)=\mathbb{Q}$. Its graded dual

$$
\mathcal{A} \bullet(F):=\bigoplus_{n=0}^{\infty} \mathcal{A}_{n}(F), \quad \mathcal{A}_{n}(F):=\left(\mathrm{U}_{-n}(F)\right)^{*}
$$

has the structure of a commutative graded Hopf algebra with a coproduct $\Delta$. One has

$$
\mathcal{L}_{\bullet}(F)=\mathcal{A}_{\bullet}(F) /\left(\mathcal{A}_{>0}(F) \cdot \mathcal{A}_{>0}(F)\right)
$$

So the elements of $\mathcal{L}_{\bullet}(F)$ are the elements of $\mathcal{A}_{\bullet}(F)$ considered modulo the sums of products of elements of positive weight.

Now let $X$ be a complex variety and denote by $\mathbb{C}(X)$ the field of rational functions on $X$. To give a first idea why the Lie coalgebra $\mathcal{L}_{\bullet}(F)$ and the Hopf algebra $\mathcal{A}_{\bullet}(F)$ are relevant to the analytic theory of polylogarithms and their generalizations, let us start with a vague statement:

\section{Any weight $n$ polylogarithm-like function $\mathcal{F}$ on $X$ gives rise to an element $\mathcal{F}^{\mathcal{M}}$ of $\mathcal{A}_{\bullet}(\mathbb{C}(X))$. Considered modulo products of such functions, it provides an element of $\mathcal{L}_{\bullet}(\mathbb{C}(X))$.}

Precisely, but using terminology which we are not going to explain here, a 'weight $n$ polylogarithm-like function $\mathcal{F}$ on $X^{\prime}$ ' is a period of a weight $n$ framed variation of mixed $\mathbb{Q}$-Hodge structures on an open part of $X$, with the Hodge weights $h^{p, q}$ being zero unless $p=q$, which is of 'geometric origin'. We call such functions Hodge-Tate periods.

It is conjectured that in passing from $\mathcal{F}$ to its motivic avatar $\mathcal{F}^{\mathcal{M}}$ we do not 'lose any information about $\mathcal{F}$ '. See [9], where the motivic avatars of multiple polylogarithms were defined, for a detailed account on the subject. The main point is this:

To know a Hodge-Tate period function $\mathcal{F}$ is the same thing as to know its motivic avatar $\mathcal{F} \mathcal{M}$. The vector space $\mathcal{A}_{n}(\mathbb{C}(X))$ is precisely the linear vector space spanned by motivic avatars of the weight $n$ Hodge-Tate period functions on open parts of $X$.

\footnotetext{
${ }^{1}$ However this is rarely the case; see [23] for the treatment of duals in the infinite-dimensional situation.
} 
The benefit of replacing $\mathcal{F}$ by $\mathcal{F}^{\mathcal{M}}$ is that the latter lie in a Hopf algebra. Since this Hopf algebra is graded by non-negative integers, its elements can be studied by applying the coproduct to them, which is expressible via similar objects of lower weight. The fundamental fact is that the kernels of the coproduct maps

$$
\Delta: \mathcal{A}_{\bullet}(\mathbb{C}(X)) \longrightarrow \otimes^{2} \mathcal{A}_{\bullet}(\mathbb{C}(X)), \quad \delta: \mathcal{L}_{\bullet}(\mathbb{C}(X)) \longrightarrow \Lambda^{2} \mathcal{L}_{\bullet}(\mathbb{C}(X))
$$

are given by constants. Therefore, taking the coproduct does not discard any essential information about the function.

\subsection{Higher Bloch groups}

So the key question is to describe the Hopf algebra $\mathcal{A}_{\bullet}(\mathbb{C}(X))$, or, equivalently, the Lie coalgebra $\mathcal{L}_{\bullet}(\mathbb{C}(X))$. The structure of $\mathcal{L}_{\bullet}(F)$ for any field $F$ is essentially predicted by the Freeness Conjecture [23]. We start from its low weight consequences.

Weight 1. First of all, one has

$$
\mathcal{L}_{1}(F)=F^{*} \otimes_{\mathbb{Z}} \mathbb{Q}
$$

Weight 2. Let us recall the definition of the Bloch group $B_{2}(F)[50,51]$. Let $\mathbb{Q}[F]$ be the $\mathbb{Q}$-vector space with basis elements $\{x\}$ for $x \in F$. Recall the cross-ratio

$$
r\left(x_{1}, x_{2}, x_{3}, x_{4}\right)=\frac{\left(x_{1}-x_{2}\right)\left(x_{3}-x_{4}\right)}{\left(x_{2}-x_{3}\right)\left(x_{1}-x_{4}\right)} .
$$

Notice the unusual normalization of the cross-ratio: $r(\infty,-1,0, x)=x$.

Given any 5 points $x_{1}, \ldots, x_{5}$ on the projective line $\mathbb{P}^{1}(F)$ over $F$, set

$$
\sum_{i=1}^{5}\left\{r\left(x_{i}, x_{i+1}, x_{i+2}, x_{i+3}\right)\right\} \in \mathbb{Q}[F] .
$$

Here the indices are considered modulo 5 .

Let $R_{2}(F)$ be the subspace generated by $\{0\}$ and the five-term relations (4.10). Over the complex numbers, (4.10) provides Abel's famous pentagon relation for the dilogarithm. Precisely, consider the Bloch-Wigner single valued version of the dilogarithm, altered by the $z \longmapsto-z$ argument change:

$$
\mathcal{L}_{2}(z):=\Im\left(\operatorname{Li}_{2}(-z)+\arg (1+z) \log |z|\right), \quad z \in \mathbb{C} .
$$

Then Abel's pentagon relation for the Bloch-Wigner dilogarithm is

$$
\sum_{i=1}^{5} \mathcal{L}_{2}\left(r\left(x_{i}, x_{i+1}, x_{i+2}, x_{i+3}\right)\right)=0 .
$$

The Bloch-Wigner function also satisfies the reality condition $\mathcal{L}_{2}(z)+\mathcal{L}_{2}(\bar{z})=0$. Any functional equation for the Bloch-Wigner function can be deduced from this and Abel's equation. Since the complex-valued dilogarithm certainly does not satisfy in general any 
reality condition, one refers to Abel's pentagon equation as the generic functional equation for the dilogarithm.

Now the Bloch group is a $\mathbb{Q}$-vector space given by the quotient

$$
\mathrm{B}_{2}(F):=\frac{\mathbb{Q}[F]}{R_{2}(F)} .
$$

In general we denote elements of $\mathrm{B}_{n}(F)$ by $\{x\}_{n}$, with $x \in F$. It can be deduced from Beilinson's conjectures [48] and Suslin's theorem [51], that one should have

$$
\mathcal{L}_{2}(F)=\mathrm{B}_{2}(F) .
$$

Set $F_{\mathbb{Q}}^{*}:=F^{*} \otimes \mathbb{Q}$. The weight 2 part of the cocommutator map (4.2) is a map

$$
\delta: \mathcal{L}_{2}(F) \rightarrow \Lambda^{2} \mathcal{L}_{1}(F) .
$$

The claim is that using the isomorphisms (4.8) and (4.13), it becomes a map

$$
\delta: \mathrm{B}_{2}(F) \longrightarrow \Lambda^{2} F_{\mathbb{Q}}^{*}, \quad\{x\}_{2} \longmapsto(1+x) \wedge x, \quad\{0\}_{2},\{-1\}_{2} \longmapsto 0 .
$$

This can also be deduced from Suslin's theorem. A non-trivial but not difficult fact to check is that the map $\{x\} \longmapsto(1+x) \wedge x$ kills the five-term relations (4.10), and thus descends to a map of the space $\mathrm{B}_{2}(F)$.

Notice that, unlike in the more traditional way to present Abel's pentagon identity, all terms in this formula appear with a plus sign. Moreover, the arguments of the pentagon equation are nothing else but the cluster $\mathcal{X}$-coordinates on the configuration space $\operatorname{Conf}_{5}\left(\mathbb{P}^{1}\right)$ (see section 6 ). In particular, this explains the origin of the non-standard normalization of the classical cross-ratio used in the definition (4.9).

Weight 3. Let us describe now the space $\mathcal{L}_{3}(F)$, following [23]. Consider the triple ratio of 6 points $\left(z_{1}, \ldots, z_{6}\right)$ in $\mathbb{P}^{2}$, given by the formula

$$
r_{3}\left(z_{1}, \ldots, z_{6}\right):=-\frac{\langle 124\rangle\langle 235\rangle\langle 316\rangle}{\langle 125\rangle\langle 236\rangle\langle 314\rangle} .
$$

Here we pick vectors $\left(l_{1}, \ldots, l_{6}\right)$ in a three-dimensional vector space $V_{3}$ projecting onto the points $\left(z_{1}, \ldots, z_{6}\right)$, and set $\langle i j k\rangle:=\omega_{3}\left(l_{i}, l_{j}, l_{k}\right)$, where $\omega_{3}$ is a volume form in $V_{3}$.

It was proved in [23] that the triple ratio $r_{3}$ plays a similar role for the trilogarithm as the classical cross-ratio in eq. (4.9) does for the dilogarithm. Precisely, consider the following single-valued version of the trilogarithm, which is the function from [52] with argument modified by the change $z \longmapsto-z$ :

$$
\mathcal{L}_{3}(z):=\Re\left(\operatorname{Li}_{3}(-z)-\operatorname{Li}_{2}(-z) \log |z|-\frac{1}{3} \log ^{2}|z| \log (1+z)\right), \quad z \in \mathbb{C} .
$$

The functional equations for the trilogarithm are provided by configurations of 7 points $\left(z_{1}, \ldots, z_{7}\right)$ in $\mathbb{C P}^{2}$. Specifically,

$$
\sum_{i=1}^{7}(-1)^{i}\left(\operatorname{Alt}_{6} \mathcal{L}_{3}\left(r_{3}\left(z_{1}, \ldots, \widehat{z}_{i}, \ldots, z_{7}\right)\right)\right)=0 .
$$


Here $\mathrm{Alt}_{6}$ stands for the skew-symmetrization of the six points $z_{1}, \ldots, \widehat{z}_{i}, \ldots, z_{7}$.

The function $\mathcal{L}_{3}(z)$ satisfies the reality equation $\mathcal{L}_{3}(z)=\mathcal{L}_{3}(\bar{z})$. Just like in the case of the Bloch-Wigner function, any functional equation for the function $\mathcal{L}_{3}(z)$ can be deduced from the reality equation and the equation (4.19). So the latter is referred to as the generic functional equation for the trilogarithm.

For an arbitrary field $F$, given any 7 points on the projective plane over $F$, consider an element

$$
\sum_{i=1}^{7}(-1)^{i}\left(\operatorname{Alt}_{6}\left\{r_{3}\left(z_{1}, \ldots, \widehat{z}_{i}, \ldots, z_{7}\right)\right\}\right) \in \mathbb{Q}[F] .
$$

Let $R_{3}(F)$ be the subspace generated by the elements $(4.20)$, where $\left(z_{1}, \ldots, z_{7}\right)$ are points in the projective plane over $F$, and $\{0\}$. Set

$$
\mathrm{B}_{3}(F):=\frac{\mathbb{Q}[F]}{R_{3}(F)} .
$$

One deduces from the work $[23,53]$ on the proof of Zagier's conjecture [52] on special values of Dedekind $\zeta$-functions at $s=3$ that one should have

$$
\mathcal{L}_{3}(F)=\mathrm{B}_{3}(F),
$$

However the nature of the triple ratio (4.17) was a mystery. It was realized much later that the triple ratio is a cluster $\mathcal{X}$-coordinate on the configuration space $\operatorname{Conf}_{3}\left(\mathbb{P}^{2}\right)$, and moreover it is one which cannot be reduced to cross-ratios of the type in eq. (4.9). We will return to this later on.

The weight 3 part of the cocommutator map (4.2) is a map ${ }^{2}$

$$
\mathcal{L}_{3}(F) \rightarrow \mathcal{L}_{2}(F) \otimes \mathcal{L}_{1}(F)
$$

It follows from [23] that, using the isomorphisms (4.8), (4.13) and (4.21), it becomes a map

$$
\delta: \mathrm{B}_{3}(F) \longrightarrow \mathrm{B}_{2}(F) \otimes F_{\mathbb{Q}}^{*}, \quad\{x\}_{3} \longmapsto\{x\}_{2} \otimes x .
$$

A quite non-trivial fact to check here is that the map $\{x\} \longmapsto\{x\}_{2} \otimes x$ kills the relations (4.20) and thus descends to a map defined on the space $\mathrm{B}_{3}(F)$, see [54].

The higher analogs of the B-groups were defined in $[23,53]$. The group $\mathcal{B}_{n}(F)$ is the quotient of the $\mathbb{Q}$-vector space $\mathbb{Q}[F]$ by the subspace of functional equations for the classical $n$-logarithm. Although the functional equations are not known explicitly in general, the subgroup they generate is defined for all $n$ inductively. Namely, consider a map

$$
\delta_{n}^{\prime}: \mathbb{Q}[F] \longrightarrow\left\{\begin{array} { l l } 
{ \mathcal { B } _ { n - 1 } ( F ) \otimes F _ { \mathbb { Q } } ^ { * } , } & { n \geq 3 } \\
{ \Lambda ^ { 2 } F _ { \mathbb { Q } } ^ { * } , } & { n = 2 , }
\end{array} \quad \{ x \} \longmapsto \left\{\begin{array}{ll}
\{x\}_{n-1} \otimes x, & n \geq 3 \\
(1-x) \wedge x, & n=2 .
\end{array}\right.\right.
$$

Now replace $F$ by the field $F(t)$ of rational functions in one variable, and take an element $\sum_{i} a_{i}\left\{f_{i}(t)\right\} \in \mathbb{Q}[F(t)]$ killed by the map $\delta_{n}^{\prime}$. We define a subspace $\mathcal{R}_{n}(F) \subset \mathbb{Q}[F]$ as the

\footnotetext{
${ }^{2}$ Here and in all that follows we use $V \otimes W$ to denote the summand in $\Lambda^{2}(V \oplus W)$ given by vectors of the form $v \otimes w-w \otimes v$, since the map $v \otimes w \in V \otimes W \longmapsto v \otimes w-w \otimes v \in \Lambda^{2}(V \oplus W)$ is injective.
} 
subspace generated by the elements $\sum_{i} a_{i}\left(\left\{f_{i}(0)\right\}-\left\{f_{i}(1)\right\}\right)$, where we added $\{\infty\}:=0$. Then we set

$$
\mathcal{B}_{n}(F):=\frac{\mathbb{Q}[F]}{\mathcal{R}_{n}(F)}
$$

The change of notation from $\mathrm{B}_{n}(F)$ to $\mathcal{B}_{n}(F)$ emphasizes that we deal with the definition where the functional equations are not known explicitly. The map $\delta_{n}^{\prime}$ induces a map

$$
\delta_{n}: \mathcal{B}_{n}(F) \longrightarrow\left\{\begin{array} { l l } 
{ \mathcal { B } _ { n - 1 } ( F ) \otimes F _ { \mathbb { Q } } ^ { * } , } & { n \geq 3 , } \\
{ \Lambda ^ { 2 } F _ { Q } ^ { * } , } & { n = 2 , }
\end{array} \quad \{ x \} _ { n } \longmapsto \left\{\begin{array}{ll}
\{x\}_{n-1} \otimes x, & n \geq 3, \\
(1-x) \wedge x, & n=2 .
\end{array}\right.\right.
$$

One has $\mathcal{B}_{n}(F)=\mathrm{B}_{n}(F)$ for $n=2,3$.

At this point one might ask whether we have $\mathcal{L}_{n}(F)=\mathcal{B}_{n}(F)$ for all $n$. It was shown in [23] that this is not the case starting with $n=4$. Since this is the case we deal with when studying two-loop amplitudes, let us discuss it in detail.

Weight 4. It is conjectured in $[23]^{3}$ that the $\mathbb{Q}$-vector space $\mathcal{L}_{4}(F)$ is an extension

$$
0 \longrightarrow \mathcal{B}_{4} \longrightarrow \mathcal{L}_{4} \longrightarrow \Lambda^{2} \mathrm{~B}_{2} \longrightarrow 0 \text {. }
$$

Here we start skipping the field $F$ in the notation.

To understand the nature of this extension, let us look at the coproduct map

$$
\delta: \mathcal{L}_{4} \longrightarrow \Lambda^{2} \mathcal{L}_{2} \bigoplus \mathcal{L}_{3} \otimes \mathcal{L}_{1}=\Lambda^{2} \mathrm{~B}_{2} \bigoplus \mathrm{B}_{3} \otimes F_{\mathbb{Q}}^{*}
$$

The map

$$
\delta_{2,2}: \mathcal{L}_{4} \longrightarrow \Lambda^{2} \mathrm{~B}_{2}
$$

in (4.28) is just a part of the coproduct. It is known to be surjective. The restriction of the coproduct to the subspace $\mathcal{B}_{4}$ in (4.28) is described as the map (4.27) for $n=4$.

One can reformulate this as follows. Given an element $l \in \mathcal{L}_{4}(F)$, one can ask whether it can be written as a sum of the (motivic) classical 4 -logarithms. If $\delta_{2,2}(l) \neq 0$, the answer is no. Indeed, the coproduct of the classical motivic 4-logarithm is given by

$$
\delta:\{x\}_{4} \longmapsto\{x\}_{3} \otimes x \in \mathrm{B}_{3}(F) \otimes F_{\mathbb{Q}}^{*}
$$

Therefore the $\mathrm{B}_{2} \wedge \mathrm{B}_{2}$-component is zero. The deeper part of the conjecture tells that if $\delta_{2,2}(l)=0$, the answer is yes. So the map (4.30) is precisely the obstruction for an element $l$ to be a sum of the (motivic) classical 4-logarithms.

In summary, one can express $\mathcal{L}_{4}$ in terms of the higher Bloch groups, which reflect properties of the classical polylogarithms, which are function of a single variable. The Freeness Conjecture tells that a similar description is expected for all $\mathcal{L}_{n}$. Here is its essential part:

\footnotetext{
${ }^{3}$ This is a conjecture about a conjectural object. The point is that the very existence of the Lie coalgebra $\mathcal{L} \bullet(F)$, although still conjectural, follows from some 'standard' conjectures in algebraic geometry. Although there is a lot of evidence for the conjecture on $\mathcal{L}_{4}(F)$, it is not known how to reduce it to any 'first principles' conjectures.
} 
Conjecture. Let Lieb. $(F)$ be the free graded Lie algebra generated by the $\mathbb{Q}$-vector spaces $\mathcal{B}_{n}(F)^{*}$ dual to $\mathcal{B}_{n}(F)$, where $n \geq 2$ and the weight of $\mathcal{B}_{n}(F)^{*}$ is $-n$. Let us denote by Lieb* $(F)$ the graded dual Lie coalgebra, graded by $n=2,3, \ldots$ Then one has

$$
\mathcal{L}_{\bullet}(F)=\operatorname{Lieb}_{\bullet}^{*}(F) .
$$

For example, when $n=1,2,3$ we cannot present $n$ as a sum of two integers $\geq 2$, and thus the Freeness Conjecture implies $\mathcal{L}_{n}=\mathcal{B}_{n}$ for $n=1,2,3$. In contrast with this, $4=2+2$, and so, besides $\mathcal{B}_{4}$, we have an extra contribution to $\mathcal{L}_{4}$ given by $\Lambda^{2} \mathrm{~B}_{2}$. It is the dual to the commutators of the weight -2 elements in the free Lie algebra Lieb.

\subsection{Symbols}

Recall the motivic Hopf algebra $\mathcal{A}_{\bullet}(F)$. One has $\mathcal{A}_{1}(F)=F_{\mathbb{Q}}^{*}$. Let $\Delta_{n-1,1}: \mathcal{A}_{n}(F) \longrightarrow$ $\mathcal{A}_{n-1}(F) \otimes \mathcal{A}_{1}(F)$ be the $(n-1,1)$-component of the coproduct. Iterating it, we get a sequence of maps

$$
\begin{aligned}
\mathcal{A}_{n}(F) & \longrightarrow \mathcal{A}_{n-1}(F) \otimes \mathcal{A}_{1}(F) \\
& \longrightarrow \mathcal{A}_{n-2}(F) \otimes \mathcal{A}_{1}(F) \otimes \mathcal{A}_{1}(F) \\
& \longrightarrow \ldots \\
& \longrightarrow \otimes^{n} \mathcal{A}_{1}(F)=\otimes^{n} F_{\mathbb{Q}}^{*} .
\end{aligned}
$$

Its composition is a map, called the symbol map:

$$
\mathrm{S}: \mathcal{A}_{n}(F) \longrightarrow \otimes^{n} F_{\mathbb{Q}}^{*}
$$

\subsection{Motivic scattering amplitudes}

The $L$-loop $n$-particle MHV motivic amplitudes in SYM theory, considered modulo products, are elements $\mathcal{A}_{n, L}^{\mathcal{M}} \in \mathcal{L}_{2 L}$. The two-loop motivic amplitudes are therefore elements

$$
\mathcal{A}_{n, 2}^{\mathcal{M}}\left(Z_{1}, \ldots, Z_{n}\right) \in \mathcal{L}_{4}(F), \quad F=\mathbb{Q}\left(Z_{1}, \ldots, Z_{n}\right)
$$

defined by a generic configuration of $n$ points $\left(Z_{1}, \ldots, Z_{n}\right)$ in $\mathbb{P}^{3}$. Therefore, as was explained above, according to the conjectural description (4.28) of $\mathcal{L}_{4}$, they can be expressed via $\mathrm{Li}_{4}(z)$ if and only if the $\Lambda^{2} \mathrm{~B}_{2}$ obstruction vanishes. This is exactly what happened in [22] where the two-loop $n=6 \mathrm{MHV}$ amplitude was calculated as a sum of classical 4-logarithms. The problem set out for us here and subsequent work is therefore:

Problem. Calculate the motivic $n$-particle two-loop MHV amplitudes for $n>6$. More specifically, this amounts to computing the coproduct

$$
\delta_{\mathcal{M}}\left(\mathcal{A}_{n, 2}^{\mathcal{M}}\left(Z_{1}, \ldots, Z_{n}\right)\right) \in \Lambda^{2} \mathrm{~B}_{2}(F) \bigoplus \mathrm{B}_{3}(F) \otimes F_{\mathbb{Q}}^{*}, \quad F=\mathbb{Q}\left(Z_{1}, \ldots, Z_{n}\right) .
$$

The coproduct determines the amplitude as a function up to a constant and products of similar functions of lower weight. Unlike the mysterious extension (4.28), which is nonsplit, the coproduct is given in terms of the groups $\mathrm{B}_{n}, n=1,2,3$, and so its calculation 
is a precise problem. Let us emphasize that due to the 'one-variable' nature of the groups $\mathrm{B}_{n}$, to write an element in $\Lambda^{2} \mathrm{~B}_{2} \oplus \mathrm{B}_{3} \otimes F_{\mathbb{Q}}^{*}$ we need a collection of functions on the configuration space $\operatorname{Conf}_{n}\left(\mathbb{P}^{3}\right)$ - the arguments of the dilogarithms and trilogarithms. The only ambiguity of such a presentation results from the functional equations they satisfy.

As we show below, these functions for the 2-loop $n$-particle MHV motivic amplitudes, where $n=6,7$, are cluster $\mathcal{X}$-coordinates on the space $\operatorname{Conf}_{n}\left(\mathbb{P}^{3}\right)$. Moreover, although functional equations do come into the picture, the ones we see for the two-loop amplitudes are also of cluster nature, and discussed in appendix B.

We would like to stress that without the motivic approach one cannot even formulate Problem 4.4- there is no way to define the coproduct just on the level of functions. Moreover, due to non-split nature of the extension (4.28), there is even no canonical way to write a general element of $\mathcal{L}_{4}$.

\section{The coproducts of two-loop MHV motivic amplitudes}

Using the symbol of the two-loop $n$-point MHV amplitude, as computed in [55], one can calculate the coproduct $\delta\left(\mathcal{A}_{n, 2}^{\mathcal{M}}\right)$ of the corresponding motivic amplitude as defined in the previous section and summarized in eq. (4.35). For $n=6$ the $\Lambda^{2} \mathrm{~B}_{2}$ component is trivial, as was noted already in [22], while the $\mathrm{B}_{3} \otimes \mathbb{C}^{*}$ part of the coproduct may be read off immediately from (3.7):

$$
\left.\delta\left(\mathcal{A}_{6,2}^{\mathcal{M}}\right)\right|_{\mathrm{B}_{3} \otimes \mathbb{C}^{*}}=\sum_{i=1}^{3}\left\{x_{i}^{+}\right\}_{3} \otimes x_{i}^{+}+\left\{x_{i}^{-}\right\}_{3} \otimes x_{i}^{-}-\frac{1}{2}\left\{v_{i}\right\}_{3} \otimes v_{i} .
$$

We defer results for general $n$ to a subsequent publication and present here explicit results only for $n=7$, since our main goal at the moment is to call the reader's attention to the same two non-trivial features that we emphasized in section 3 . The first feature is that the entry $z$ appearing inside each $\{z\}_{2}$ or $\{z\}_{3}$ is a single cross-ratio (rather than, as might have been the case, some arbitrary algebraic function of cross-ratios); the second feature is that of the thousands of such cross-ratios one can form at $n=7$, only a small handful actually appear in the motivic amplitudes. The structure of the results presented here will be extensively discussed in subsequent sections.

\subsection{The $\Lambda^{2} \mathrm{~B}_{2}$ component for $n=7$}

We find that the $\Lambda^{2} \mathrm{~B}_{2}$ component of $\delta\left(\mathcal{A}_{7,2}^{\mathcal{M}}\right)$ can be expressed as

$$
\begin{aligned}
\sum_{\text {dihedral }}\left(\left\{\frac{\langle 7 \times 1,2 \times 3,4 \times 5\rangle}{\langle 127\rangle\langle 345\rangle}\right\}_{2} \wedge\left\{\frac{\langle 2 \times 3,4 \times 6,7 \times 1\rangle}{\langle 167\rangle\langle 234\rangle}\right\}_{2}\right. \\
\left.+\frac{1}{2}\left\{\frac{\langle 312\rangle\langle 347\rangle}{\langle 371\rangle\langle 342\rangle}\right\}_{2} \wedge\left\{\frac{\langle 713\rangle\langle 746\rangle}{\langle 716\rangle\langle 734\rangle}\right\}_{2}\right)+ \text { parity conjugate }
\end{aligned}
$$

where the sum indicates that one should sum over the dihedral group acting on the particle labels, resulting in a total of 42 distinct terms (the $1 / 2$ in front of the second term is a symmetry factor). 
It is important to note that this expression is of course not unique. There are two kinds of ambiguities: first of all, there are dilogarithm identities which hold at the level of $\mathrm{B}_{2}$ and are a consequence of Abel's pentagon relation (4.12). One slightly non-trivial, but easily checked, example is

$$
\begin{aligned}
0= & -\left\{\frac{\langle 127\rangle\langle 156\rangle\langle 345\rangle}{\langle 157\rangle\langle 1 \times 2,3 \times 4,5 \times 6\rangle}\right\}_{2}+\left\{\frac{\langle 127\rangle\langle 256\rangle\langle 345\rangle}{\langle 257\rangle\langle 1 \times 2,3 \times 4,5 \times 6\rangle}\right\}_{2} \\
& +\left\{-\frac{\langle 567\rangle\langle 1 \times 2,3 \times 4,5 \times 6\rangle}{\langle 256\rangle\langle 1 \times 7,3 \times 4,5 \times 6\rangle}\right\}_{2}-\left\{-\frac{\langle 127\rangle\langle 345\rangle\langle 567\rangle}{\langle 157\rangle\langle 2 \times 7,3 \times 4,5 \times 6\rangle}\right\}_{2} \\
& -\left\{\frac{\langle 571\rangle\langle 562\rangle}{\langle 512\rangle\langle 567\rangle}\right\}_{2} .
\end{aligned}
$$

Such identities relate different, but equivalent, expressions for the $\Lambda^{2} \mathrm{~B}_{2}$ component which in general have different numbers of terms.

Secondly there is ambiguity in writing (5.2) due to the trivial identities

$$
\{x\}_{2} \wedge\{y\}_{2}=-\{1 / x\}_{2} \wedge\{y\}_{2}=\{1 / x\}_{2} \wedge\{1 / y\}_{2}=-\{x\}_{2} \wedge\{1 / y\}_{2}
$$

which preserve the number of terms.

Given these ambiguities one may wonder about the value in providing any explicit formula such as (5.2). Is there some invariant way of presenting the $\Lambda^{2} \mathrm{~B}_{2}$ part of the coproduct of this amplitude, without having to commit any particular representation? To phrase this question in language familiar to physicists: if we think about the equations (5.3) and (5.4) as generating some kind of gauge transformations, then what is the gauge-invariant content of the $\Lambda^{2} \mathrm{~B}_{2}$ component of this coproduct?

We pose this question here merely as a teaser; to answer it fully requires the mathematical machinery to be built up in section 6 . Nevertheless we will also tease the reader here with the answer: the 42 terms in (5.2) are naturally in correspondence with certain quadrilateral faces of the $E_{6}$ Stasheff polytope, and this is the shortest manifestly symmetric (dihedral + parity) representative with this property.

\subsection{The $\mathrm{B}_{3} \otimes \mathbb{C}^{*}$ component for $n=7$}

In order to save space, we will make use of the dihedral symmetry and invariance under rescaling of the MHV amplitude to write only the two independent $\mathrm{B}_{3}$ components necessary to express the full answer. First, for the $\langle 124\rangle$ component of $\mathbb{C}^{*}$ we find the $\mathrm{B}_{3}$ element

$$
\begin{aligned}
& \left\{\frac{\langle 127\rangle\langle 256\rangle\langle 345\rangle}{\langle 257\rangle\langle 1 \times 2,3 \times 4,5 \times 6\rangle}\right\}_{3}+\left\{\frac{\langle 125\rangle\langle 234\rangle\langle 567\rangle}{\langle 257\rangle\langle 1 \times 2,3 \times 4,5 \times 6\rangle}\right\}_{3} \\
& -\left\{\frac{\langle 127\rangle\langle 234\rangle\langle 345\rangle\langle 567\rangle}{\langle 257\rangle\langle 347\rangle\langle 1 \times 2,3 \times 4,5 \times 6\rangle}\right\}_{3}+\left\{\frac{\langle 124\rangle\langle 157\rangle}{\langle 127\rangle\langle 145\rangle}\right\}_{3}+\left\{-\frac{\langle 127\rangle\langle 254\rangle\langle 516\rangle}{\langle 124\rangle\langle 256\rangle\langle 517\rangle}\right\}_{3} \\
& -\left\{\frac{\langle 214\rangle\langle 257\rangle}{\langle 217\rangle\langle 245\rangle}\right\}_{3}+\left\{\frac{\langle 412\rangle\langle 435\rangle}{\langle 415\rangle\langle 423\rangle}\right\}_{3}-\left\{\frac{\langle 412\rangle\langle 437\rangle}{\langle 417\rangle\langle 423\rangle}\right\}_{3}-\left\{-\frac{\langle 147\rangle\langle 452\rangle\langle 516\rangle}{\langle 142\rangle\langle 456\rangle\langle 517\rangle}\right\}_{3} \\
& -\left\{-\frac{\langle 247\rangle\langle 453\rangle\langle 526\rangle}{\langle 243\rangle\langle 456\rangle\langle 527\rangle}\right\}_{3}+\left\{\frac{\langle 412\rangle\langle 457\rangle}{\langle 417\rangle\langle 425\rangle}\right\}_{3}-\left\{\frac{\langle 512\rangle\langle 547\rangle}{\langle 517\rangle\langle 524\rangle}\right\}_{3}+\left\{\frac{\langle 712\rangle\langle 745\rangle}{\langle 715\rangle\langle 724\rangle}\right\}_{3}
\end{aligned}
$$




$$
\begin{aligned}
& -\left\{\frac{\langle 413\rangle\langle 457\rangle}{\langle 417\rangle\langle 435\rangle}\right\}_{3}+\left\{\frac{\langle 423\rangle\langle 457\rangle}{\langle 427\rangle\langle 435\rangle}\right\}_{3}-\left\{-\frac{\langle 241\rangle\langle 453\rangle\langle 527\rangle}{\langle 243\rangle\langle 457\rangle\langle 521\rangle}\right\}_{3} \\
& +\left\{-\frac{\langle 241\rangle\langle 473\rangle\langle 725\rangle}{\langle 243\rangle\langle 475\rangle\langle 721\rangle}\right\}_{3}+\left\{\frac{\langle 512\rangle\langle 567\rangle}{\langle 517\rangle\langle 526\rangle}\right\}_{3}-\left\{-\frac{\langle 254\rangle\langle 576\rangle\langle 721\rangle}{\langle 256\rangle\langle 571\rangle\langle 724\rangle}\right\}_{3} \\
& -\left\{\frac{\langle 514\rangle\langle 567\rangle}{\langle 517\rangle\langle 546\rangle}\right\}_{3}+\left\{-\frac{\langle 452\rangle\langle 576\rangle\langle 741\rangle}{\langle 456\rangle\langle 571\rangle\langle 742\rangle}\right\}_{3}-\left\{\frac{\langle 524\rangle\langle 567\rangle}{\langle 527\rangle\langle 546\rangle}\right\}_{3} \\
& +\left\{-\frac{\langle 453\rangle\langle 576\rangle\langle 742\rangle}{\langle 456\rangle\langle 572\rangle\langle 743\rangle}\right\}_{3} .
\end{aligned}
$$

Secondly, for $\langle 125\rangle$ (this is symmetric under $1 \leftrightarrow 2,7 \leftrightarrow 3,6 \leftrightarrow 4$ ) we find

$$
\begin{aligned}
-\left\{\frac{\langle 157\rangle\langle 234\rangle}{\langle 1 \times 2,3 \times 4,5 \times 7\rangle}\right\}_{3}-\left\{\frac{\langle 123\rangle\langle 457\rangle}{\langle 1 \times 2,3 \times 4,5 \times 7\rangle}\right\}_{3}-\left\{\frac{\langle 127\rangle\langle 134\rangle\langle 567\rangle}{\langle 167\rangle\langle 1 \times 2,3 \times 4,5 \times 7\rangle}\right\}_{3} \\
+\left\{\frac{\langle 127\rangle\langle 234\rangle\langle 567\rangle}{\langle 267\rangle\langle 1 \times 2,3 \times 4,5 \times 7\rangle}\right\}_{3}+\left\{\frac{\langle 123\rangle\langle 345\rangle\langle 567\rangle}{\langle 356\rangle\langle 1 \times 2,3 \times 4,5 \times 7\rangle}\right\}_{3} \\
-\left\{\frac{\langle 124\rangle\langle 345\rangle\langle 567\rangle}{\langle 456\rangle\langle 1 \times 2,3 \times 4,5 \times 7\rangle}\right\}_{3}-\left\{\frac{\langle 123\rangle\langle 145\rangle}{\langle 125\rangle\langle 134\rangle}\right\}_{3}-2\left\{\frac{\langle 125\rangle\langle 167\rangle}{\langle 127\rangle\langle 156\rangle}\right\}_{3} \\
+\left\{\frac{\langle 412\rangle\langle 435\rangle}{\langle 415\rangle\langle 423\rangle}\right\}_{3}+\left\{\frac{\langle 512\rangle\langle 534\rangle}{\langle 514\rangle\langle 523\rangle}\right\}_{3}-\left\{-\frac{\langle 231\rangle\langle 354\rangle\langle 526\rangle}{\langle 234\rangle\langle 356\rangle\langle 521\rangle}\right\}_{3} \\
+\left\{-\frac{\langle 241\rangle\langle 453\rangle\langle 526\rangle}{\langle 243\rangle\langle 456\rangle\langle 521\rangle}\right\}_{3}+\left\{\frac{\langle 523\rangle\langle 546\rangle}{\langle 526\rangle\langle 534\rangle}\right\}_{3}+(1 \leftrightarrow 2,7 \leftrightarrow 3,6 \leftrightarrow 4) .
\end{aligned}
$$

The full $\mathrm{B}_{3} \otimes \mathbb{C}^{*}$ part of the coproduct of the two-loop $n=7$ MHV motivic amplitude is assembled in terms of these two building blocks as

$$
\sum_{\text {dihedral }}\left[(\text { eq. }(5.5)) \otimes \frac{\langle 124\rangle\langle 567\rangle}{\langle 127\rangle\langle 456\rangle}+\frac{1}{2}(\text { eq. }(5.6)) \otimes \frac{\langle 125\rangle\langle 167\rangle\langle 234\rangle}{\langle 123\rangle\langle 127\rangle\langle 456\rangle}\right] .
$$

Let us comment briefly on the action of parity (defined and discussed in detail in appendix A) on eq. (5.7). If we apply parity to the first term in eq. (5.7) and then the permutation $(1,7,6,5,4,3,2)$, the $\mathbb{C}^{*}$ term is unchanged. The corresponding $B_{3}$ terms are identical up to a 40-term $\mathrm{Li}_{3}$ identity, of the type discussed in appendix B. The parity invariance of the second term in eq. (5.7) is easier to prove: applying parity followed by the permutation $(4,5,6,7,1,2,3)$ leaves both the $\mathbb{C}^{*}$ term and the corresponding $\mathrm{B}_{3}$ part invariant. Therefore in this case the parity invariance is manifest, without requiring any $\mathrm{Li}_{3}$ identities. We note with interest that the aforementioned 40-term identity is the only nontrivial $\mathrm{Li}_{3}$ identity which plays a role in elucidating the structure of this amplitude.

\section{Cluster coordinates and cluster algebras}

Having presented concrete results of the calculation of the coproduct for the two-loop $n=7 \mathrm{MHV}$ amplitude, we now turn to a second main theme of the paper: establishing the connection between the coproduct of motivic amplitudes and cluster algebras. This section is aimed primarily at physicists since most of this material is a review of fairly 
well-known mathematical facts, with a focus on the intended application to $\operatorname{Conf}_{n}\left(\mathbb{P}^{3}\right)$, the space on which scattering amplitudes live. We start with a short introduction to cluster algebras, which were discovered and first developed in a series of papers [25, 26] by Fomin and Zelevinsky. The configuration space $\operatorname{Conf}_{n}\left(\mathbb{P}^{k-1}\right)$ of $n$ points in $\mathbb{P}^{k-1}$ has the structure of a cluster Poisson variety [24] - a structure closely related to cluster algebras. Our aim in this section is to guide the reader quickly to an understanding of what cluster variables are, and how they may be systematically constructed via a process called mutation. Cluster coordinates come in two types, referred to as cluster $\mathcal{A}$-coordinates for Grassmannians and cluster $\mathcal{X}$-coordinates for the configuration spaces $\operatorname{Conf}_{n}\left(\mathbb{P}^{k-1}\right)$.

To guide the reader, let us specify the spaces where different types of cluster coordinates live. Recall (see section 2) that the Grassmannian $\operatorname{Gr}(k, n)$ is birationally isomorphic to the configuration space of vectors $\operatorname{Conf}_{n}(k)$. The latter projects onto the space of projective configurations $\operatorname{Conf}_{n}\left(\mathbb{P}^{k-1}\right)$ :

$$
\operatorname{Gr}(k, n) \stackrel{\sim}{\longrightarrow} \operatorname{Conf}_{n}(k) \stackrel{\pi}{\longrightarrow} \operatorname{Conf}_{n}\left(\mathbb{P}^{k-1}\right) .
$$

Cluster $\mathcal{A}$-coordinates live naturally on the Plücker cone $\widetilde{\operatorname{Gr}}(k, n)$ over the Grassmannian rather than on the Grassmannian itself. This cone can be identified birationally with the configuration space $\widetilde{\operatorname{Conf}_{n}}(k)$ of $n$ vectors modulo the action of the group $S L_{k}$ rather than $G L_{k}$. Abusing terminology, one often refers to them as coordinates on the Grassmannian. Cluster $\mathcal{X}$-coordinates live naturally on the smaller space $\operatorname{Conf}_{n}\left(\mathbb{P}^{k-1}\right)$-its dimension is $n$ less then that of the $\widetilde{\operatorname{Conf}_{n}}(k)$. They describe a collection of log-canonical Darboux coordinate systems for the natural cyclic invariant Poisson structure on the space $\operatorname{Conf}_{n}\left(\mathbb{P}^{k-1}\right)$. Using the canonical projection

$$
\widetilde{\pi}: \widetilde{\operatorname{Gr}}(k, n) \longrightarrow \operatorname{Conf}_{n}\left(\mathbb{P}^{k-1}\right)
$$

one can lift the cluster $\mathcal{X}$-coordinates on $\operatorname{Conf}_{n}\left(\mathbb{P}^{k-1}\right)$, and express them as monomials of the cluster variables in the Grassmannian cluster algebra. One cannot extend, however, the Poisson structure to the cluster algebra without breaking the cyclic invariance. Notice that the cluster algebra structure itself on $\widetilde{\operatorname{Conf}}_{n}(k)$ is (twisted) cyclic invariant. The cyclic invariance is a crucial feature of the planar scattering amplitudes. So it is important that both Grassmannian cluster algebra and the cluster Poisson structure on $\operatorname{Conf}_{n}\left(\mathbb{P}^{k-1}\right)$ are cyclic invariant.

\subsection{Introduction and definitions}

We can informally define cluster algebras as follows: they are commutative algebras constructed from distinguished generators (called cluster variables) grouped into non-disjoint sets of constant cardinality (called clusters), which are constructed iteratively from an initial cluster by an operation called mutation. The number of variables in a cluster is called the rank of the cluster algebra.

A simple example is the $A_{2}$ cluster algebra defined by the following data:

- cluster variables: $a_{m}$ for $m \in \mathbb{Z}$, subject to $a_{m-1} a_{m+1}=1+a_{m}$ 
- rank: 2

- clusters: $\left\{a_{m}, a_{m+1}\right\}$ for $m \in \mathbb{Z}$

- initial cluster: $\left\{a_{1}, a_{2}\right\}$

- mutation: $\left\{a_{m-1}, a_{m}\right\} \rightarrow\left\{a_{m}, a_{m+1}\right\}$.

Using the exchange relation $a_{m-1} a_{m+1}=1+a_{m}$ one sees that

$$
a_{3}=\frac{1+a_{2}}{a_{1}}, \quad a_{4}=\frac{1+a_{1}+a_{2}}{a_{1} a_{2}}, \quad a_{5}=\frac{1+a_{1}}{a_{2}}, \quad a_{6}=a_{1}, \quad a_{7}=a_{2} .
$$

Therefore, the sequence $a_{m}$ is periodic with period five and the number of cluster variables is finite.

When expressing the cluster variables $a_{m}$ in terms of $a_{1}$ and $a_{2}$, we encounter two interesting features. First, the denominators of the cluster variables are always monomials. In general the structure of an algebra is such that one might expect the cluster variables to be more general rational functions of the initial cluster variables, but in fact the denominator is always a monomial. This is known as the 'Laurent phenomenon' (see [25, 56]). A second feature is that, conjecturally, the numerator of each cluster variable, expressed in terms of the original cluster variables, is always a polynomial with positive integer coefficients.

Some cluster algebras may be defined in terms of another piece of mathematical machinery: quivers. A quiver is an oriented graph, and in the following we restrict to connected, finite quivers without loops (arrows with the same origin and target) and two-cycles (pairs of arrows going in opposite directions between two nodes).

Given a quiver and a choice of some node $k$ on that quiver we can define a new quiver obtained by mutating at node $k$. The new quiver is obtained by applying the following operations on the initial quiver:

- for each path $i \rightarrow k \rightarrow j$, add an arrow $i \rightarrow j$,

- reverse all arrows on the edges incident with $k$,

- and remove any two-cycles that may have formed.

The mutation at $k$ is an involution; when applied twice in succession at the same node we come back to the original quiver.

Quivers of the special type under consideration are in one-to-one correspondence with skew-symmetric matrices defined as

$$
b_{i j}=(\# \text { arrows } i \rightarrow j)-(\# \text { arrows } j \rightarrow i) .
$$

Since at most one of the terms above is nonvanishing, $b_{i j}=-b_{j i}$. Under a mutation at node $k$ the matrix $b$ transforms to $b^{\prime}$ given by

$$
b_{i j}^{\prime}= \begin{cases}-b_{i j}, & \text { if } k \in\{i, j\}, \\ b_{i j}, & \text { if } b_{i k} b_{k j} \leq 0, \\ b_{i j}+b_{i k} b_{k j}, & \text { if } b_{i k}, b_{k j}>0, \\ b_{i j}-b_{i k} b_{k j}, & \text { if } b_{i k}, b_{k j}<0\end{cases}
$$


If we start with a quiver with $n$ nodes and associate to each node $i$ a variable $a_{i}$, we can use the skew-symmetric matrix $b$ to define a mutation relation at the node $k$ by

$$
a_{k} a_{k}^{\prime}=\prod_{i \mid b_{i k}>0} a_{i}^{b_{i k}}+\prod_{i \mid b_{i k}<0} a_{i}^{-b_{i k}}
$$

with the understanding that an empty product is set to one. The mutation at $k$ changes $a_{k}$ to $a_{k}^{\prime}$ defined by eq. (6.5) and leaves the other cluster variables unchanged.

To illustrate these ideas we note that the initial cluster of the $a_{2}$ cluster algebra can be expressed by the quiver $a_{1} \rightarrow a_{2}$. Then, a mutation at $a_{1}$ replaces it by $a_{1}^{\prime}=\frac{1+a_{2}}{a_{1}} \equiv a_{3}$ and reverses the arrow. A mutation at $a_{2}$ replaces it by $a_{2}^{\prime}=\frac{1+a_{1}}{a_{2}} \equiv a_{5}$ and preserves the direction of the arrow.

\subsection{Cluster Poisson varieties}

These are defined using the same combinatorial skeleton: quivers and mutations of quivers. We assign now to the nodes of the quiver variables $\left\{x_{i}\right\}$ which mutate according to the following rule:

$$
x_{i}^{\prime}= \begin{cases}x_{k}^{-1}, & i=k, \\ x_{i}\left(1+x_{k}^{\mathrm{sgn} b_{i k}}\right)^{b_{i k}}, & i \neq k .\end{cases}
$$

There is a natural Poisson bracket on the cluster $\mathcal{X}$-coordinates. It is enough to define the Poisson bracket between the $\mathcal{X}$-coordinates in a given cluster, for which it is given in terms of the antisymmetric matrix $b_{i j}$ defined in (6.3) by

$$
\left\{x_{i}, x_{j}\right\}=b_{i j} x_{i} x_{j}
$$

An important property of this Poisson bracket is that it is invariant under mutations, in the sense that

$$
\left\{x_{i}^{\prime}, x_{j}^{\prime}\right\}=b_{i j}^{\prime} x_{i}^{\prime} x_{j}^{\prime}
$$

whenever $x_{i}^{\prime}, x_{j}^{\prime}$ and $b_{i j}^{\prime}$ are obtained from $x_{i}, x_{j}$ and $b_{i j}$ by a mutation.

Given a quiver described by the matrix $b$, the cluster $\mathcal{A}$ - and $\mathcal{X}$-coordinates can be related as follows:

$$
x_{i}=\prod_{j} a_{j}^{b_{i j}}
$$

This relation is preserved under mutations (changing, of course, the $b_{i j}$-matrix).

We would like to stress that the cluster $\mathcal{A}$ - and $\mathcal{X}$-coordinates live on different spaces of the same dimension, denoted $\mathcal{A}$ and $\mathcal{X}$. Indeed, they are parametrized by the same set, the set of nodes of the corresponding quiver. Formula (6.9) is just a coordinate way to express a canonical map of spaces $p: \mathcal{A} \rightarrow \mathcal{X}$. The dimension of the fibers of this map is the corank of the matrix $b$.

A sequence of cluster mutations can result in reproducing the original quiver, while providing a non-trivial transformation of the cluster $\mathcal{A}$ - and $\mathcal{X}$-coordinates. Such transformations form a group, introduced in [24] under the name cluster modular group. 


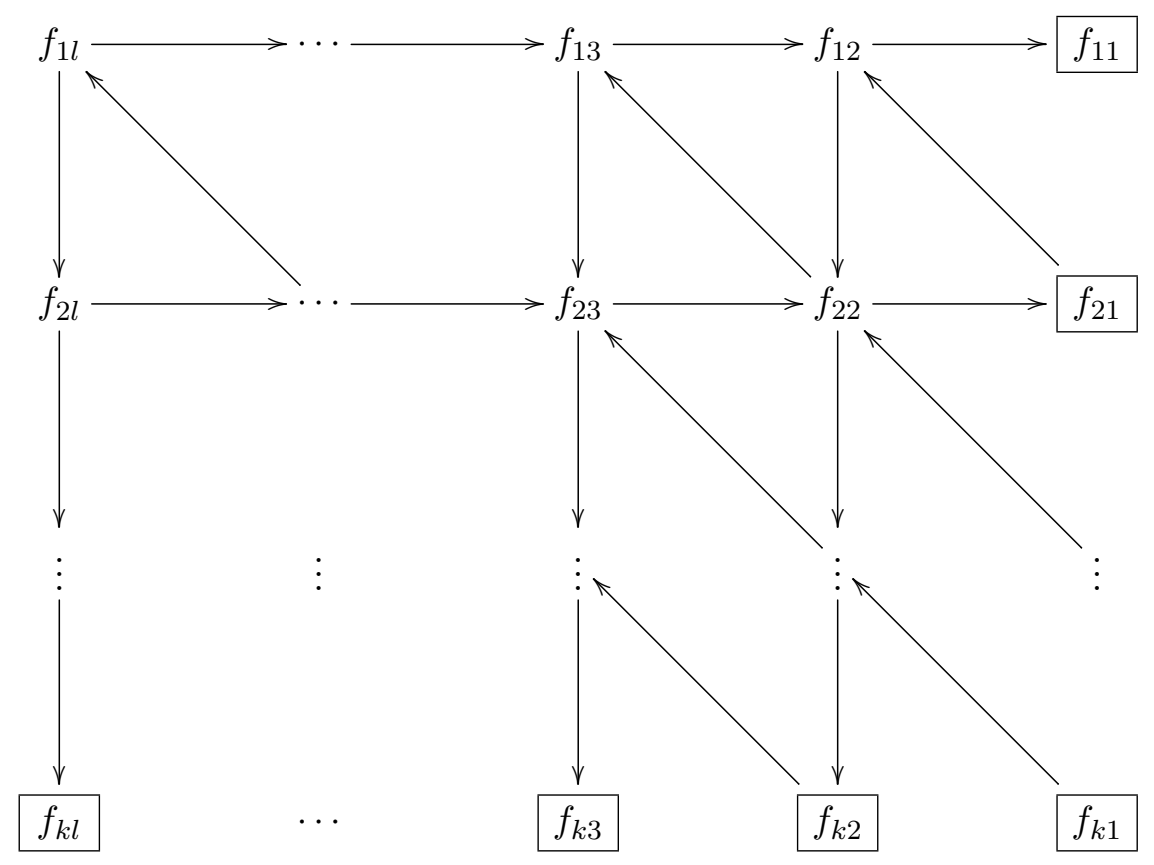

Figure 1. The initial quiver for the $\operatorname{Gr}(k, n)$ cluster algebra (see refs. [58, 59]).

\subsection{Grassmannian cluster algebras and cluster Poisson spaces $\operatorname{Conf}_{n}\left(\mathbb{P}^{k-1}\right)$}

In our application we are interested in a special class of cluster algebras called cluster algebras of geometric type. They are also described by quivers, but some of the nodes are special and called frozen nodes. Edges connecting two frozen nodes are not allowed, ${ }^{4}$ and we also do not allow mutations on the frozen nodes. The variables associated to the frozen nodes are called coefficients instead of cluster variables (and the rank of the algebra is equal only to the number of unfrozen nodes). We define the principal part of such a quiver to be the quiver obtained by erasing the frozen nodes as well as all edges which connect them to any of the non-frozen nodes. When drawing these special kinds of quivers, we will indicate each frozen node by placing its label inside a box.

We now review the cluster algebras of geometric type which arise from the Grassmannian $\operatorname{Gr}(k, n)$ [57]. The Plücker coordinates $\left\langle i_{1} \ldots i_{k}\right\rangle$, being the minors obtained by computing the determinant of the indicated columns $i_{1} \ldots i_{k}$ of a matrix representative of a point in $\operatorname{Gr}(k, n)$, are examples of $\mathcal{A}$-coordinates.

The Plücker coordinates satisfy the relation

$$
\langle i, j, I\rangle\langle k, l, I\rangle=\langle i, k, I\rangle\langle j, l, I\rangle+\langle i, l, I\rangle\langle j, k, I\rangle,
$$

where $I$ is a multi-index with $k-2$ entries, which bears a resemblance to the exchange relation shown in eq. (6.5). Indeed the cluster algebra for $\operatorname{Gr}(k, n)$ is constructed by starting with an initial cluster whose variables are certain Plücker coordinates. The operation of

\footnotetext{
${ }^{4}$ There is a different approach, advocated in [24], where half-edges between frozen variables are not only allowed, but in fact play a crucial role in the amalgamation construction: building bigger cluster structures from the smaller ones.
} 
mutation generates additional Plücker coordinates, as well as other, more complicated, cluster $\mathcal{A}$-coordinates. For general $k$ and $n$ and with $l=n-k$, the appropriate initial quiver is given in ref. [58] (this construction is also reviewed in ref. [59]) and shown in figure 1, where

$$
f_{i j}=\left\{\begin{array}{ll}
\frac{\langle i+1, \ldots, k, k+j, \ldots, i+j+k-1\rangle}{\langle 1, \ldots, k\rangle}, & i \leq l-j+1, \\
\frac{\langle 1, \ldots, i+j-l-1, i+1, \ldots, k, k+j, \ldots, n\rangle}{\langle 1, \ldots, k\rangle}, & i>l-j+1
\end{array} .\right.
$$

The boxes identify the frozen variables while the rest of the variables are unfrozen.

In order to obtain the quivers we will use below we need to make one last change to the quiver above. We rescale all the coordinates, frozen and unfrozen, by $\langle 1, \ldots, k\rangle$. This produces a frozen variable $\langle 1, \ldots, k\rangle$ which connects to the node labeled by $f_{1 l}$ by an ingoing arrow. After this modification all the unfrozen nodes of the initial quiver have an equal number of ingoing and outgoing arrows.

The simplest nontrivial example is that of $\operatorname{Gr}(2,5)$, which is relevant for configurations of five points in $\mathbb{P}^{1}$. In this case the initial quiver is simply

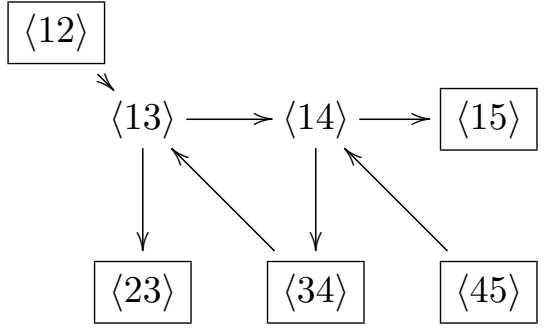

where each node is labeled by its $\mathcal{A}$-coordinate. In this case it is easy to check that successive mutations on the two unfrozen nodes, in any order, generate only five distinct quivers. The algebra so generated is nothing but the $A_{2}$ algebra defined at the beginning of section 6.1. The name of the algebra comes from the fact that the principal part of this initial quiver is the same as the Dynkin diagram of the $A_{2}$ Lie algebra.

Let us use this example to calculate $\mathcal{X}$-coordinates. There is one such coordinate associated to each unfrozen node $k$, expressed by taking the product of the $\mathcal{A}$-coordinates on the nodes connected to $k$ by an incoming arrow, divided by the product of the $\mathcal{A}$ coordinates on the nodes connected to $k$ by an outgoing arrow. So in the $A_{2}$ quiver shown above the $\mathcal{X}$-coordinates associated to the nodes $\langle 13\rangle$ and $\langle 14\rangle$ are $\langle 12\rangle\langle 34\rangle /\langle 23\rangle\langle 14\rangle$ and $\langle 13\rangle\langle 45\rangle /\langle 34\rangle\langle 15\rangle$ respectively. Cluster $\mathcal{A}$-coordinates are not invariant under rescaling of the individual vectors in a $\operatorname{Gr}(k, n)$ matrix, but the $\mathcal{X}$-coordinates are. Therefore only the latter are good coordinates on the quotient $\operatorname{Gr}(k, n) /\left(\mathbb{C}^{*}\right)^{n-1}=\operatorname{Conf}_{n}\left(\mathbb{P}^{k-1}\right)$ and are hence appropriate objects to appear in motivic amplitudes.

To connect with the above general discussion of cluster Poisson varieties and $\mathcal{X}$ coordinates, in this example we use only the unfrozen part of the quiver to build the $\mathcal{X}$-coordinates. This leads to a reduced cluster $\mathcal{X}$-space $\mathcal{X}^{\prime}$, and the map described coordinately in (6.9) reduces to a surjective map $p^{\prime}: \mathcal{A} \rightarrow \mathcal{X}^{\prime}$ describing the projection $\widetilde{\mathrm{Gr}}(k, n) \rightarrow \operatorname{Conf}_{n}\left(\mathbb{P}^{k-1}\right)$. 
The two simplest examples relevant to SYM theory scattering amplitudes are those for 6 or 7 points in $\mathbb{P}^{3}$ (or, equivalently, in $\mathbb{P}^{1}$ or $\mathbb{P}^{2}$, respectively). For the former it is evident from figure 1 that the principal part of the quiver is the same as the $A_{3}$ Dynkin diagram. For the latter the initial quiver is slightly more complicated:

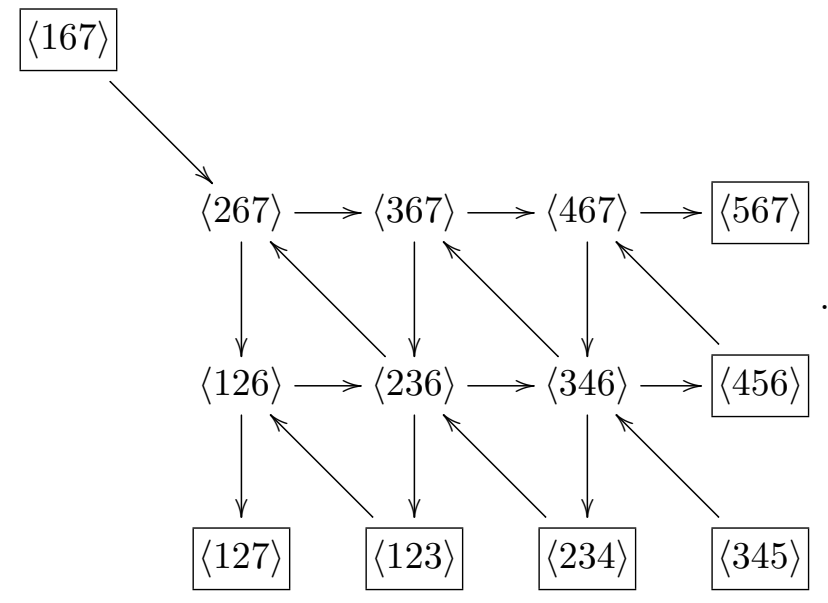

If we label the nodes occupied initially by $\langle 267\rangle,\langle 367\rangle,\langle 467\rangle,\langle 126\rangle,\langle 236\rangle,\langle 346\rangle$ by numbers 1 through 6 , then after a sequence of mutations at nodes $4,3,2,5,1,4,3,4,6$, the principal part of the quiver is brought into the form of the $E_{6}$ Dynkin diagram ${ }^{5}$

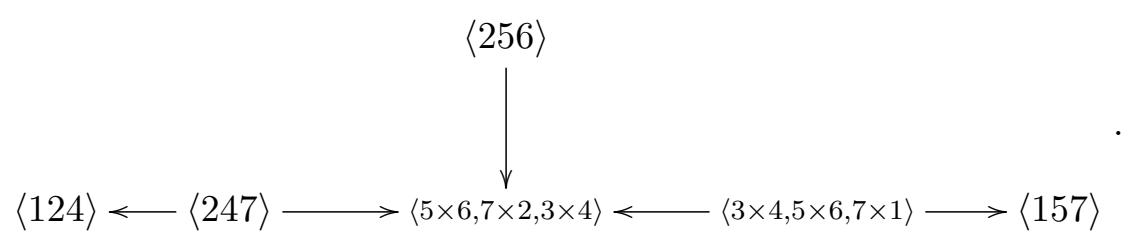

Therefore the $\operatorname{Gr}(3,7)$ cluster algebra is also called the $E_{6}$ algebra.

In [26] Fomin and Zelevinsky showed that a cluster algebra is of finite type (i.e., it has a finite number of cluster variables) if there exists a sequence of mutations which turns the principal part of its quiver into the Dynkin diagram of some classical Lie algebra. However, if the principal part of the quiver contains a subgraph which is an affine Dynkin diagram, then the cluster algebra is of infinite type.

In ref. [57], Scott classified all the Grassmannian cluster algebras of finite type. As discussed in section 2, the relevant Grassmannian for scattering amplitudes in SYM theory is $\operatorname{Gr}(4, n)$, for $n \geq 6$. If $n=6$ we need $\operatorname{Gr}(4,6)=\operatorname{Gr}(2,6)$ which is of finite type $A_{3}$. If $n=7$ we need $\operatorname{Gr}(4,7)=\operatorname{Gr}(3,7)$ which is again of finite type $E_{6}$. However, starting at $n=8$ the relevant cluster algebras are not of finite type anymore. This indicates that there are infinitely many different $\mathcal{A}$-coordinates which could appear in the symbol of these amplitudes, and infinitely many different $\mathcal{X}$-coordinates could appear in their coproduct.

Besides the usual Plücker determinants, mutations also lead in general to more complicated $\mathcal{A}$-coordinates. For example, with $\operatorname{Gr}(4,8)$ one encounters

$$
\langle 12(345) \cap(678)\rangle \equiv\langle 1345\rangle\langle 2678\rangle-\langle 2345\rangle\langle 1678\rangle .
$$

\footnotetext{
${ }^{5}$ If we order them in the same way as in the initial cluster, the $\mathcal{A}$-coordinates after this sequence of mutations are $\langle 3 \times 4,5 \times 6,7 \times 1\rangle,\langle 256\rangle,\langle 124\rangle,\langle 247\rangle,\langle 5 \times 6,7 \times 2,3 \times 4\rangle,\langle 157\rangle$.
} 
As discussed in section 2, the notation with $\cap$ emphasizes the following geometrical fact: the composite bracket $\langle 12(345) \cap(678)\rangle$ vanishes whenever the points 1 and 2 are coplanar with the projective line $(345) \cap(678)$ obtained by intersecting the two projective planes (345) and (678).

One miraculous feature of the mutations is that the denominator can always be canceled by the numerator, after using Plücker identities. Therefore, the $\mathcal{A}$-coordinates always end up being polynomials in the Plücker coordinates. This is an analog of the Laurent phenomenon mentioned above. An example which appears for $\operatorname{Gr}(4,8)$ is

$$
\frac{\langle 1237\rangle\langle 1245\rangle\langle 1678\rangle+\langle 1278\rangle\langle 45(671) \cap(123)\rangle}{\langle 1267\rangle}=\langle 45(781) \cap(123)\rangle .
$$

Here the left-hand side is the raw expression obtained for a certain $\mathcal{A}$-coordinate following some mutation, while the right-hand side is a simplified expression where the denominator has been canceled by applying a Plücker relation to the numerator in order to pull out all overall factor of $\langle 1267\rangle$.

Both of these types of non-Plücker $\mathcal{A}$-coordinates, eqs. (6.16) and (6.15), appear in the symbol of the two-loop $n=8 \mathrm{MHV}$ amplitude [55], and the simpler ones of the type in eq. (6.16) appear already for $n=7$-indeed the long formulas in section 5 are littered with these composite brackets (though expressed there in the $\mathbb{P}^{2}$ language).

However, since the number of $\mathcal{A}$-coordinates is infinite for $\operatorname{Gr}(4,8)$, mutations must eventually generate even more exotic $\mathcal{A}$-coordinates. A still relatively simple example is

$$
-\langle(123) \cap(345),(567) \cap(781)\rangle .
$$

This quantity vanishes when the lines $(123) \cap(345)$ and $(567) \cap(781)$ intersect, which is equivalent to saying that the lines $(345) \cap(567)$ and $(781) \cap(123)$ intersect. But there are even more complicated $\mathcal{A}$-coordinates such as

$$
\begin{aligned}
\langle 1246\rangle\langle 1256\rangle\langle 1378\rangle\langle 3457\rangle-\langle 1246\rangle\langle 1257\rangle\langle 1378\rangle\langle 3456\rangle & \\
-\langle 1246\rangle\langle 1278\rangle\langle 1356\rangle\langle 3457\rangle+\langle 1278\rangle\langle 1257\rangle\langle 1346\rangle\langle 3456\rangle & +\langle 1236\rangle\langle 1278\rangle\langle 1457\rangle\langle 3456\rangle .
\end{aligned}
$$

Neither of these complicated quantities appears as an entry in the symbol of the $n=8$ MHV amplitude at two loops, but we know of no reason why they cannot appear at higher loops. It would be extremely interesting to understand more about these algebras and their relation to amplitudes.

One final, important comment has to do with cyclic symmetry, which is an exact symmetry of MHV amplitudes (and of all super-amplitudes). Notice that the initial quivers we have been using break the cyclic symmetry of the configuration of points. In order to see that the cyclic symmetry is preserved we need to show that by mutations one can reach another quiver whose labels are permuted by one unit. For the case of $\operatorname{Gr}(3,7)$ described above, this cannot be done in fewer than six mutations, since all the unfrozen $\mathcal{A}$-coordinates need to change. Indeed one can easily show that after mutating in the nodes which are initially labeled by $\langle 126\rangle,\langle 267\rangle,\langle 236\rangle,\langle 367\rangle,\langle 346\rangle$ and $\langle 467\rangle$, we obtain the cluster with 
the node labels shifted by one $\langle 123\rangle \rightarrow\langle 234\rangle$, etc. This proves the cyclic symmetry for $\operatorname{Gr}(3,7)$. It is not hard to imagine that a similar procedure can be applied in the general $\operatorname{Gr}(k, n)$ case, but we do not provide a complete proof of cyclic symmetry here.

\subsection{Generalized Stasheff polytopes}

In this section we review the connection [33] between cluster algebras and certain polytopes, including the generalized Stasheff polytope or associahedron [34, 35]. Many additional details and examples may also be found in [60].

The unfrozen nodes of a cluster algebra of rank $r$ can be taken to be the vertices of an $r$-1-simplex. A $k$-simplex is a generalization of the notion of a triangle and can be defined as a convex hull of its $k+1$ vertices. A 0 -simplex is a point, a 1 -simplex is a line, a 2 -simplex is a triangle, a 3 -simplex is a tetrahedron, and so on.

If we take a subset of $l+1$ vertices of the $k+1$ vertices of a $k$-simplex we can form an $l$ simplex which is called a face of the $k$-simplex. The number of $l$-faces of a $k$-simplex is $\left(\begin{array}{c}k+1 \\ l+1\end{array}\right)$.

When doing a mutation in a cluster algebra of rank $r$, one of the $\mathcal{A}$-coordinates changes while the other $r-1$ stay unchanged. They define an $r-2$-face of the initial $r-1$-simplex. Therefore, after mutation we obtain a new $r$-1-simplex which shares an $r$-2-face with the initial simplex. We can glue them along this face to form an $r$-1-dimensional polytope. For cluster algebras of finite type, by doing all possible mutations, we can build a polytope out of finitely many simplices.

In this language, the $\mathcal{X}$-coordinates of the cluster algebra correspond to $r-2$-faces of the $r$-1-simplex. The number of such faces is $\left(\begin{array}{c}r \\ r-1\end{array}\right)=r$, which equal to the rank of the algebra. Under mutations, one of the $\mathcal{X}$-coordinates transforms to its inverse while the others transform in a more complicated way. So more properly we should associate to each $r-2$-face the pair consisting of an $\mathcal{X}$-coordinate and its inverse.

The dual of the polytope we have described is a generalized Stasheff polytope (or generalized associahedron, for a reason we will describe in the following section) associated to the cluster algebra. It is a theorem which is deduced from [25], that for any cluster algebras, the faces are always either quadrilaterals or pentagons. For example, the Stasheff polytope associated to $\operatorname{Gr}(2,6)$ has 3 quadrilateral faces and 6 pentagonal faces, while the $\operatorname{Gr}(3,7)$ polytope has 1785 quadrilaterals and 1071 pentagons.

\subsection{Poisson bracket and generalized Stasheff polytopes}

There is a simple connection between the Poisson bracket and the geometry of the Stasheff polytope, which plays an important role in elucidating the structure of motivic amplitudes.

Two $\mathcal{X}$-coordinates $x_{1}, x_{2}$ have zero Poisson bracket if the Stasheff polytope has some quadrilateral face containing both $x_{1}$ and $x_{2}$ at each node in the configuration




where the dots stand for other $\mathcal{X}$-coordinates (some of which may overlap between some, but not all, of the four corners). In such a case the variables $x_{1}, x_{2}$ form a closed $A_{1} \times A_{1}$ subalgebra. Moving left-to-right or up-to-down is accomplished by mutating on $x_{1}$ or $x_{2}$, respectively.

Two $\mathcal{X}$-coordinates $x_{1}, x_{2}$ have Poisson bracket ${ }^{6} \pm 1$ if they form a closed $A_{2}$ subalgebra. In this case the Stasheff polytope has some pentagonal face containing $x_{1}$ and $x_{2}$ in the configuration

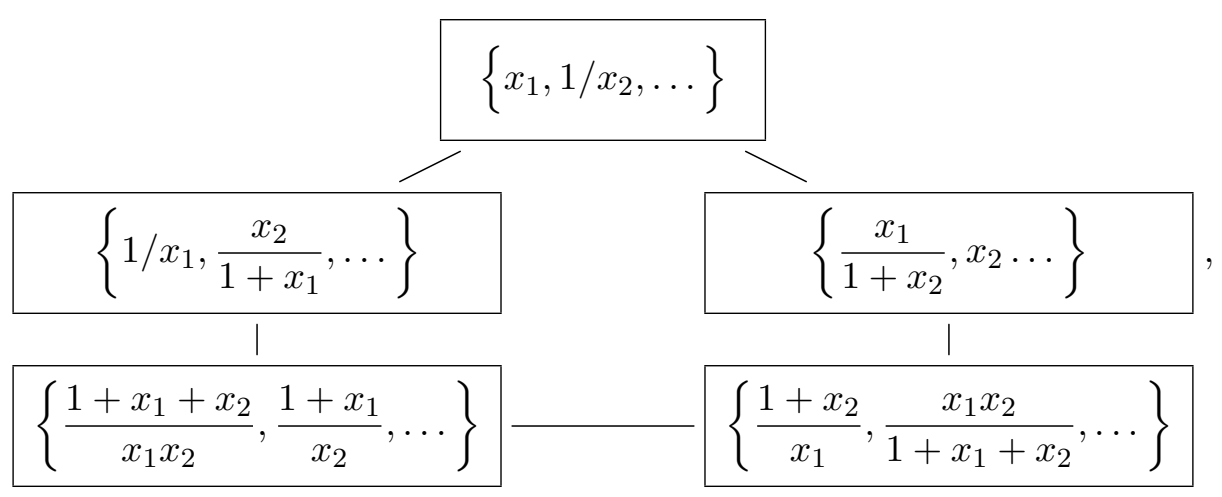

in which case the variables $x_{1}, x_{2}$ form an $A_{2}$ subalgebra.

\subsection{Parity invariance}

In this section we show that the parity operation (reviewed in appendix A) is an element of the cluster modular group. Specifically, using the identities in appendix A, we verify that the parity transform of any quiver is related by a sequence of mutations to the original quiver. This implies that the set of cluster $\mathcal{X}$-coordinates is closed under parity. It would be very interesting to see if the rest of the cluster modular group plays some role, or has a nice interpretation when acting on motivic amplitudes.

Of course, in simple cases like six points in $\mathbb{P}^{3}$ the parity invariance of the set of cluster $\mathcal{X}$-coordinates can be explicitly checked by enumerating all of them. However, due to the large number of cluster coordinates, this is much more difficult for seven points and it is impossible for more than seven points since then the cluster algebras are of infinite type.

For six points in $\mathbb{P}^{3}$ the initial quiver is shown in figure 2(a). Parity amounts to replacing $\langle i j k l\rangle \rightarrow[i j k l]$. The angle brackets $\langle i j k l\rangle$ are invariants made up of twistors $Z_{i}, Z_{j}, Z_{k}, Z_{k}$ whereas the square brackets $[i, j, k, l]$ are invariants made up of dual twistors $W_{i}, W_{j}, W_{k}, W_{k}$. Dual twistors can be written in terms of twistors $\operatorname{as}^{7} W_{i}=Z_{i-1} \wedge Z_{i} \wedge Z_{i+1}$.

\footnotetext{
${ }^{6}$ Here and in the following, when we say that two cluster $\mathcal{X}$ coordinates have Poisson bracket \pm 1 we mean that the Poisson bracket of their logarithms is \pm 1 .

${ }^{7}$ This is often written as $W_{i}=\frac{Z_{i-1} \wedge Z_{i} \wedge Z_{i+1}}{\langle i-1 i\rangle\langle i i+1\rangle}$ such that $W_{i}$ and $Z_{i}$ scale with opposite weight. The two-brackets $\langle i j\rangle$ are defined by choosing an arbitrary line $I$ (also called 'infinity twistor') and setting $\langle i j\rangle=\langle I i j\rangle$. When constructing cross-ratios these two-brackets cancel out so in the following we will not keep track of them.
} 




(a)

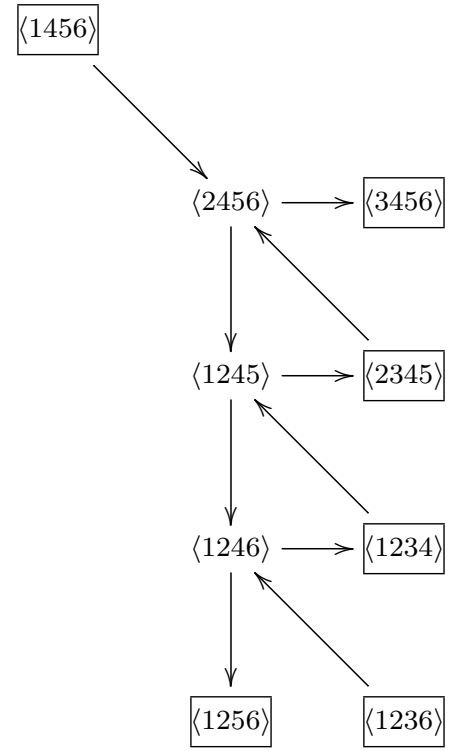

(b)

Figure 2. The initial quiver (a) for $\operatorname{Gr}(4,6)$ and its parity conjugate (b).

Then we rewrite the $[i j k l]$ in terms of angle brackets, as follows

$$
\begin{aligned}
& {[1235]=\langle 6123\rangle\langle 1234\rangle\langle 2456\rangle, \quad[1245]=\langle 6123\rangle\langle 3456\rangle\langle 1245\rangle,} \\
& {[1345]=\langle 2345\rangle\langle 3456\rangle\langle 6124\rangle, \quad[1234]=\langle 6123\rangle\langle 1234\rangle\langle 2345\rangle,} \\
& \text { cyclic permutations of [1234]. }
\end{aligned}
$$

The $\mathcal{X}$-coordinates of the quiver in figure $2(\mathrm{~b})$ generate parity conjugates of the $\mathcal{X}$ coordinates of the quiver in figure 2(a). This quiver can be obtained from the initial quiver by mutations, but with opposite directions of the arrows. Switching the direction of all arrows replaces all the cross-ratios by their inverses. This does not change the set of cluster coordinates since if a cross-ratio is a cluster $\mathcal{X}$-coordinate then its inverse is also a cluster $\mathcal{X}$-coordinate.

We should note here that the parity transformation is, up to signs, the same as shifting all the points by three. For example, $\langle 1234\rangle \rightarrow\langle 1456\rangle,\langle 1235\rangle \rightarrow\langle 2456\rangle$.

The seven-point case is a bit more complicated. Here also we start with the initial quiver in figure $3(\mathrm{a})$ to which we apply parity $\langle i j k l\rangle \rightarrow[i j k l]$. Let us focus on the $\mathcal{X}$ coordinate sitting at the node labeled by $\langle 1235\rangle$ in figure $3(\mathrm{a})$. It is given by $\frac{\langle 1234\rangle\langle 1256\rangle}{\langle 1236\rangle\langle 1245\rangle}$.

Using equations similar to the ones we used above for six points, we can write the parity conjugate of the quiver $\mathcal{X}$-coordinate at node $\langle 1235\rangle$ as

$$
\frac{\langle 1234\rangle\langle 1256\rangle}{\langle 1236\rangle\langle 1245\rangle} \rightarrow \frac{[1234][1256]}{[1236][1245]}=\frac{\langle 1256\rangle\langle 2345\rangle\langle 4567\rangle}{\langle 1245\rangle\langle 2567\rangle\langle 3456\rangle} .
$$

The parity conjugated cross-ratio is the same as the inverse cross-ratio sitting at the opposite corner, at $\langle 2456\rangle$ in the partner quiver in figure 3(b). Each of the unfrozen variables has 




(a)

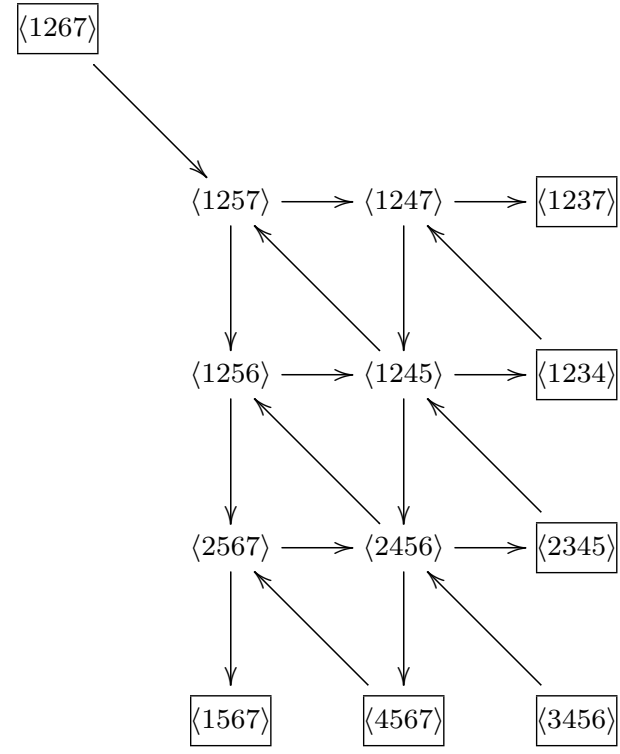

(b)

Figure 3. The initial quiver (a) for $\operatorname{Gr}(4,7)$ and its partner (b) which makes the parity conjugation property manifest.

a correspondent among the unfrozen variables of the partner quiver. The $\mathcal{X}$-coordinates of nodes which are in correspondence are inverse and parity conjugate to one another. The correspondence between nodes is defined as follows: the unfrozen nodes fit in a rectangular pattern. Flip this rectangular pattern along the vertical and the horizontal. After these flips the pattern of unfrozen nodes fits over the pattern of unfrozen nodes of the partner quiver (note that after superposition all the arrows point in the opposite directions after this sequence of operations). For example, the correspondence between the nodes in figures 3(a), 3(b) is the following: $\langle 1235\rangle \leftrightarrow\langle 2456\rangle,\langle 1245\rangle \leftrightarrow\langle 1245\rangle,\langle 1256\rangle \leftrightarrow\langle 1256\rangle$, $\langle 1236\rangle \leftrightarrow\langle 2567\rangle,\langle 1345\rangle \leftrightarrow\langle 1247\rangle,\langle 1456\rangle \leftrightarrow\langle 1257\rangle$.

Now we can show that the set of cluster $\mathcal{X}$-coordinates is closed under parity conjugation. First, it is easy to show that the quiver in figure 3(b) can be obtained from the quiver in figure 3(a) after four mutations. Another way to show that the two quivers can be obtained from one another by mutations is to notice that the quiver in figure 3(a) can be transformed to the quiver in figure $3(\mathrm{~b})$ by a dihedral transformation of external data $1 \leftrightarrow 2,3 \leftrightarrow 7,4 \leftrightarrow 6$. Then the conclusion follows from the dihedral symmetry of the cluster algebra. So they generate the same cluster algebra. Moreover, for every sequence of mutations in the quiver in figure 3(a), we can perform the same sequence of mutations in the corresponding nodes of the partner quiver in figure 3(b) and we obtain the inverses of parity conjugate $\mathcal{X}$-coordinates. This analysis can be extended without difficulty to the general case of cluster algebras $G(4, n)$. 


\subsection{Cluster algebras and the positive Grassmannian}

The positive Grassmannian is defined as the subspace of the real Grassmannian for which the ordered Plücker coordinates are all positive (see also appendix A for more details):

$$
\operatorname{Gr}^{+}(k, n)=\left\{\left(c_{1} \cdots c_{n}\right) \in \operatorname{Gr}(k, n, \mathbb{R}):\left\langle c_{a_{1}} \cdots c_{a_{k}}\right\rangle>0 \text { whenever } a_{1}<\cdots<a_{k}\right\} .
$$

The mutation relation (6.5) clearly respects positivity: if the $\mathcal{A}$-coordinates in the initial cluster are all chosen to be positive, then all subsequently generated $\mathcal{A}$-coordinates, in every cluster, will continue to be positive. The same trivially holds for $\mathcal{X}$-coordinates since they are just products of $\mathcal{A}$-coordinates.

It is manifest that set of positive configurations of points on $\mathbb{P}^{3}$ constitutes a subspace of what physicists call the Euclidean region, in which scattering amplitudes are expected to be smooth, real-valued functions. This is in strong accord with the main slogan of [32], though we are talking here about positivity in the external kinematic data, rather than in the Grassmannian of internal (i.e., loop integration) variables. It seems clear that the full power of positivity has not yet been unleashed.

By construction, any cluster $\mathcal{X}$-coordinate $x$ has the property that $1+x$ factors into a product of $\mathcal{A}$-coordinates. We have found empirically that positivity allows for a quick criterion to go the other way around: suppose we have identified some cross-ratio $r$ for which $1+r$ so factors; how do we determine whether or not $r$ is a cluster $\mathcal{X}$-coordinate? The answer is simply to evaluate the triple

$$
\{r,-1-r,-1-1 / r\}
$$

at a random point in the positive Grassmannian. In all of our experience to date, one of these three quantities will be positive and the other two negative; the positive one is an $\mathcal{X}$-coordinate and the other two are not. Certainly this criterion is valid for $n=6,7$ where we can enumerate all such possibilities, and it has held true at higher $n$ in all cases we have looked at. However in the infinite-dimensional algebras we cannot exclude the possibility that there might exist some sufficiently complicated cross-ratio $r$ for which $1+r$ factorizes, yet no member of the above list is an $\mathcal{X}$-coordinate. Let us note also that for a given cross-ratio $r$, different elements of the above list may be $\mathcal{X}$-coordinates with respect to different orderings of the external points.

\section{Cluster coordinates and motivic analysis for $n=6,7$}

We now have built up all of the machinery we need in order to carry out a full motivic analysis of the two-loop $n=6,7 \mathrm{MHV}$ amplitudes. To that end we begin this section with a detailed discussion of the cluster coordinates and Stasheff polytopes for $\operatorname{Gr}(2,6)$ and $\operatorname{Gr}(3,7)$.

\subsection{Clusters and coordinates for $\operatorname{Gr}(2,6)$}

Beginning with the initial quiver for $\operatorname{Gr}(2,6)$, we can generate all of the clusters and their $\mathcal{A}$ - and $\mathcal{X}$-coordinates by successively mutating at various nodes. The $A_{3}$ cluster algebra 


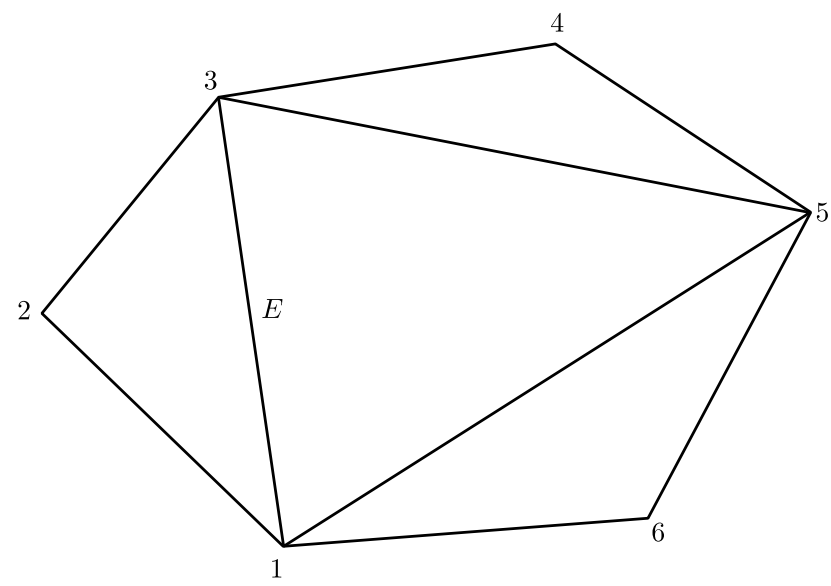

Figure 4. A triangulation $T$ of the hexagon. One of the edges of the triangulation is marked by $E$.

generated in this manner has a total of $15 \mathcal{A}$-coordinates, which are the standard Plücker coordinates $\langle i j\rangle$ on $\operatorname{Gr}(2,6)$. The six coordinates with $i$ and $j$ adjacent (mod 6$)$ are frozen, while the remaining nine are unfrozen.

However, in the special case of $\operatorname{Gr}(2, n)$ cluster algebras the mutations can also be given a simple geometric interpretation which we now describe. The discussion in this section follows ref. [24].

We start with a configuration of $n$ points in $\mathbb{P}^{1}$ with coordinates $z_{i}, i=1, \ldots, n$ and we fix a cyclic ordering. To these points we associate a convex polygon, with each vertex of the polygon labeled by one coordinate $z_{i}$. Then, consider a complete triangulation $T$ of this polygon, as in figure 4 .

A triangulation $T$ and a diagonal $E$ of that triangulation uniquely determine a quadrilateral for which $E$ is a diagonal. The points in $\mathbb{P}^{1}$ corresponding to the vertices of this quadrilateral have a cross-ratio in $\mathbb{P}^{1}$. For example, to the diagonal $E$ in figure 4 we associate the cross-ratio

$$
r(3,5,1,2)=r(1,2,3,5) \equiv \frac{\left(z_{1}-z_{2}\right)\left(z_{3}-z_{5}\right)}{\left(z_{2}-z_{3}\right)\left(z_{1}-z_{5}\right)} .
$$

Note that the vertices of the quadrilateral are read in the same order as the cyclic order of the $n$ points, starting at one of the points incident with the diagonal $E$. Note that it doesn't matter which of the two points incident with $E$ we start with, due to the identity

$$
r(k, l, i, j)=r(i, j, k, l) .
$$

Moreover, if we read the list of vertices in the opposite order, we obtain the inverse crossratio since

$$
r(i, l, k, j)=\frac{1}{r(i, j, k, l)} .
$$

Now we introduce a function $b$ which associates to a pair of diagonals in a triangulation a number which is 0 or \pm 1 . Two diagonals in a triangulation are called adjacent if they 


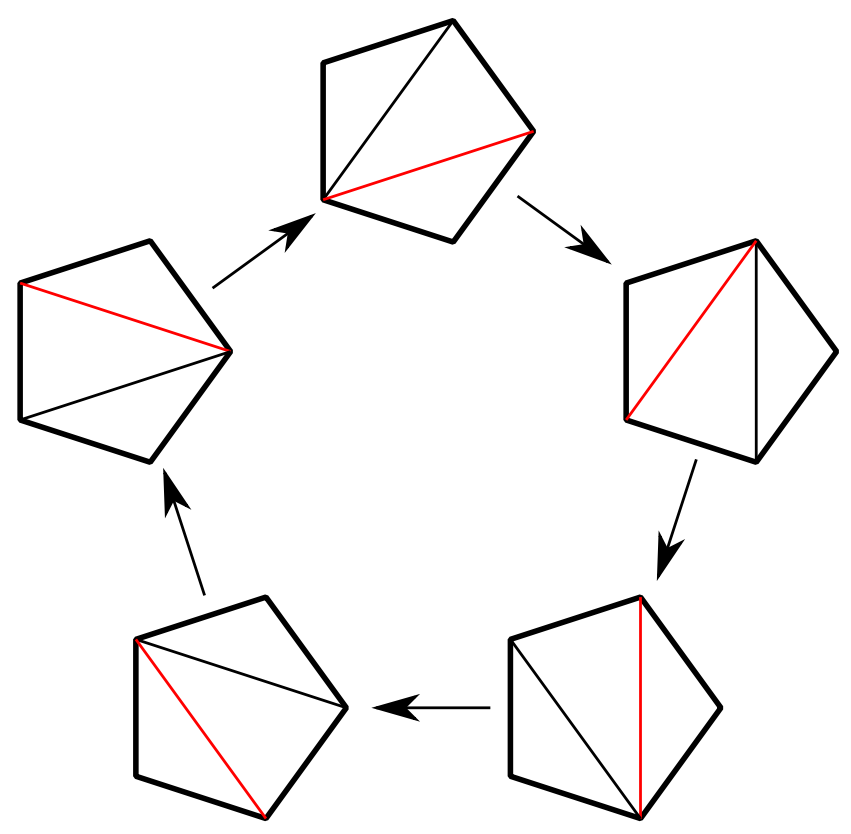

Figure 5. A sequence of mutations for five points. At each step the side colored in red gets flipped.

are the sides of one of the triangles of the triangulation. If two diagonals $E$ and $F$ are not adjacent we set $b_{E F}=0$. If $E$ and $F$ are adjacent we set $b_{E F}=1$ if $E$ comes before $F$ when listing the diagonals at $E \cap F$ in anticlockwise order. Otherwise we set $b_{E F}=-1$.

Now we can define cluster transformations (or mutations). The starting point is a triangulation, to which we can associate a set of cross-ratios, as described above (one cross-ratio for each diagonal). A cluster transformation is obtained by picking one of the diagonals and replacing it with the other diagonal in the same quadrilateral. A sequence of such mutations is represented in figure 5, where after five steps we reach the original configuration.

It is not hard to show that the effect of a mutation on the diagonal labeled by $k$ on the cross-ratios $X_{j}$ corresponding to the other diagonals is given by

$$
X_{i}^{\prime}=\mu_{k} X_{i}= \begin{cases}X_{k}^{-1}, & i=k, \\ X_{i}\left(1+X_{k}^{\operatorname{sgn}\left(b_{i k}\right)}\right)^{b_{i k},}, & i \neq k .\end{cases}
$$

This is the same as eq. (6.6). As we noted before, flipping the diagonal produces the inverse of the initial cross-ratio. The mutation in eq. (7.4) reproduces this. Also, the cross-ratios corresponding to diagonals non-adjacent to the diagonal being flipped remain unchanged. This is obvious since in this case $b_{i k}=0$.

Now let us specialize to the case $n=6$ of interest. A hexagon admits 14 distinct complete triangulations, each having three diagonals. These correspond to the 14 different clusters, each with three $\mathcal{X}$ coordinates. Out of these 42 are 15 distinct coordinates (as mentioned before we never count both $x$ and $1 / x$ separately). Nine of these ratios were already displayed in eq. (3.4); the remaining six have not been given a name in previous 
literature on $n=6$ scattering amplitudes since they do not appear in the two-loop MHV amplitude. For completeness let us list here all $15 \mathcal{X}$-coordinates

$$
\begin{aligned}
v_{1} & =r(3,5,6,2), & v_{2} & =r(1,3,4,6), & v_{3} & =r(5,1,2,4), \\
x_{1}^{+} & =r(2,3,4,1), & x_{2}^{+} & =r(6,1,2,5), & & x_{3}^{+}=r(4,5,6,3), \\
x_{1}^{-} & =r(1,4,5,6), & x_{2}^{-} & =r(5,2,3,4), & x_{3}^{-} & =r(3,6,1,2), \\
e_{1} & =r(1,2,3,5), & e_{2} & =r(2,3,4,6), & e_{3} & =r(3,4,5,1), \\
e_{4} & =r(4,5,6,2), & e_{5} & =r(5,6,1,3), & & e_{6}=r(6,1,2,4),
\end{aligned}
$$

in terms of the $\mathbb{P}^{1}$ cross-ratio defined in eq. (3.5).

Out of the 45 possible cross-ratios of the form $r(i, j, k, l)$, the $15 \mathcal{X}$-coordinates are special in that they are precisely those in which the points $i, j, k, l$ come in cyclic order. The three most well-known cross-ratios which are not cluster $\mathcal{X}$-coordinates are the ones known in the literature as

$$
u_{1}=-r(3,6,5,2), \quad u_{2}=-r(1,4,3,6), \quad u_{3}=-r(5,2,1,4) .
$$

These are related to cluster $\mathcal{X}$-coordinates by $u_{i}=1 /\left(1+v_{i}\right)$.

\subsection{The generalized Stasheff polytope for $\operatorname{Gr}(2,6)$}

Let us now discuss the geometry of the Stasheff polytope for the $A_{3}$ cluster algebra detailed in the previous section. In this case each cluster is in correspondence with a 2 -simplex, or a triangle. There are 14 clusters, to each of which corresponds a triangle. We can label each triangle by the three $\mathcal{A}$-coordinates which appear on its vertices:

$$
\begin{array}{llll}
\langle 13\rangle,\langle 14\rangle,\langle 15\rangle, & \langle 14\rangle,\langle 15\rangle,\langle 24\rangle, & \langle 13\rangle,\langle 15\rangle,\langle 35\rangle, & \langle 13\rangle,\langle 14\rangle,\langle 46\rangle, \\
\langle 15\rangle,\langle 24\rangle,\langle 25\rangle, & \langle 14\rangle,\langle 24\rangle,\langle 46\rangle, & \langle 15\rangle,\langle 25\rangle,\langle 35\rangle, & \langle 13\rangle,\langle 35\rangle,\langle 36\rangle, \\
\langle 13\rangle,\langle 36\rangle,\langle 46\rangle, & \langle 24\rangle,\langle 25\rangle,\langle 26\rangle, & \langle 24\rangle,\langle 26\rangle,\langle 46\rangle, & \langle 25\rangle,\langle 26\rangle,\langle 35\rangle, \\
\langle 26\rangle,\langle 35\rangle,\langle 36\rangle, & \langle 26\rangle,\langle 36\rangle,\langle 46\rangle . & &
\end{array}
$$

These triangles fit together in a polytope with 14 triangular faces, shown in figure 6 . The polytope has 9 vertices given by the non-frozen $\mathcal{A}$-coordinates $\langle 13\rangle,\langle 14\rangle,\langle 15\rangle,\langle 24\rangle,\langle 25\rangle$, $\langle 26\rangle,\langle 35\rangle,\langle 36\rangle$ and $\langle 46\rangle$, and 21 edges.

All faces are triangles, but there are two different types of vertices: $\langle 14\rangle,\langle 25\rangle$ and $\langle 36\rangle$ have valence four (they are incident with four edges) while the other six vertices have valence five. The polytope has the topology of a sphere as can be confirmed by computing the Euler characteristic $\chi=V-E+F=9-21+14=2$.

Now recall that to each edge of the polytope we can associate a pair consisting of an $\mathcal{X}$-coordinate and its inverse. Let us take a closer look at the $\mathcal{X}$-coordinates corresponding to the edges incident on the two kinds of vertices. In order for the association between $\mathcal{X}$-coordinates and edges to be one-to-one, we need to pick an orientation. Consider for example the valence 4 vertex shown in figure (7(a)). As we go around it we encounter the cross-ratios

$$
\frac{\langle 14\rangle\langle 23\rangle}{\langle 12\rangle\langle 34\rangle}, \quad \frac{\langle 14\rangle\langle 56\rangle}{\langle 16\rangle\langle 45\rangle}, \quad \frac{\langle 12\rangle\langle 34\rangle}{\langle 14\rangle\langle 23\rangle}, \quad \frac{\langle 16\rangle\langle 45\rangle}{\langle 14\rangle\langle 56\rangle} .
$$




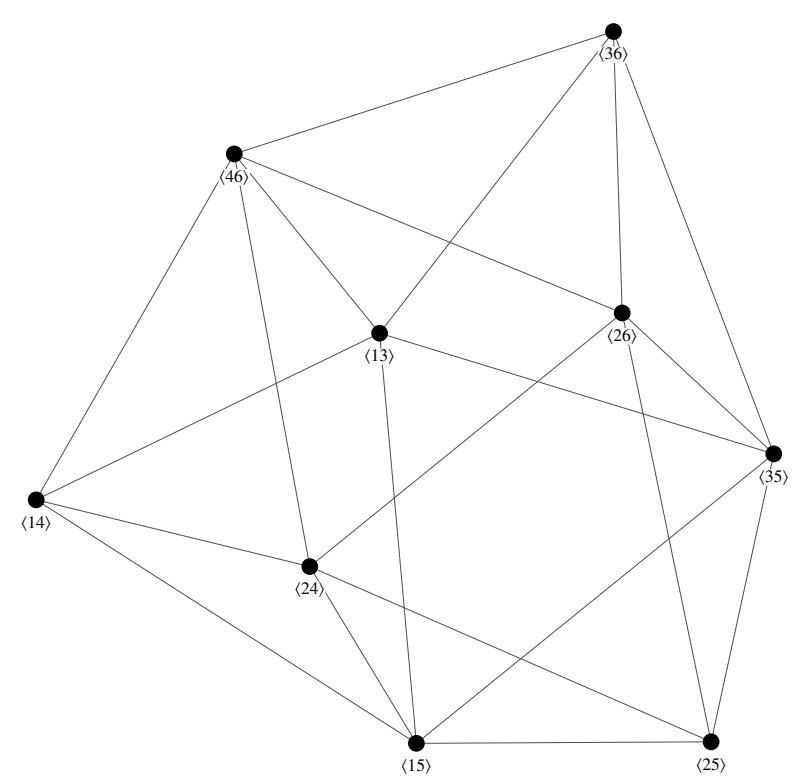

Figure 6. The polytope obtained by gluing together the triangles associated to clusters of the $\operatorname{Gr}(2,6)$ (i.e., $\left.A_{3}\right)$ cluster algebra.

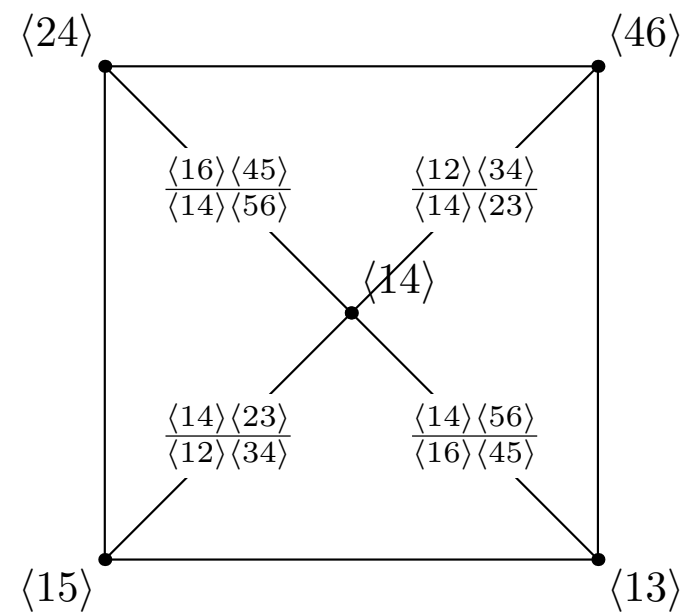

(a)

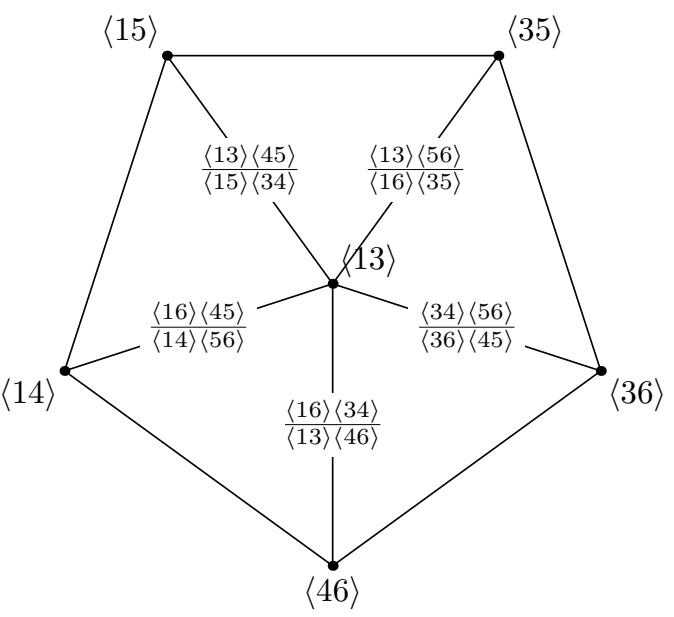

(b)

Figure 7. The cross-ratios ( $\mathcal{X}$-coordinates) around a valence 4 vertex (a) and a valence 5 vertex (b) of the polytope (figure 6 ) associated to the $\operatorname{Gr}(2,6)$ cluster algebra. For clarity we have omitted the crucial overall minus sign in front of each $\mathcal{X}$-coordinate.

The third cross-ratio is an inverse of the first while the fourth is an inverse of the second. Therefore, the cluster coordinates are the same as for the $A_{1} \times A_{1}$ cluster algebra. This is the dual of the statement shown in eq. (6.19).

On the other hand, if we consider for example the valence 5 vertex shown in figure $7(\mathrm{~b})$, the corresponding list of cross-ratios is

$$
\frac{\langle 13\rangle\langle 45\rangle}{\langle 15\rangle\langle 34\rangle}, \quad \frac{\langle 13\rangle\langle 56\rangle}{\langle 16\rangle\langle 35\rangle}, \quad \frac{\langle 34\rangle\langle 56\rangle}{\langle 36\rangle\langle 45\rangle}, \quad \frac{\langle 16\rangle\langle 34\rangle}{\langle 13\rangle\langle 46\rangle}, \quad \frac{\langle 16\rangle\langle 45\rangle}{\langle 14\rangle\langle 56\rangle} .
$$




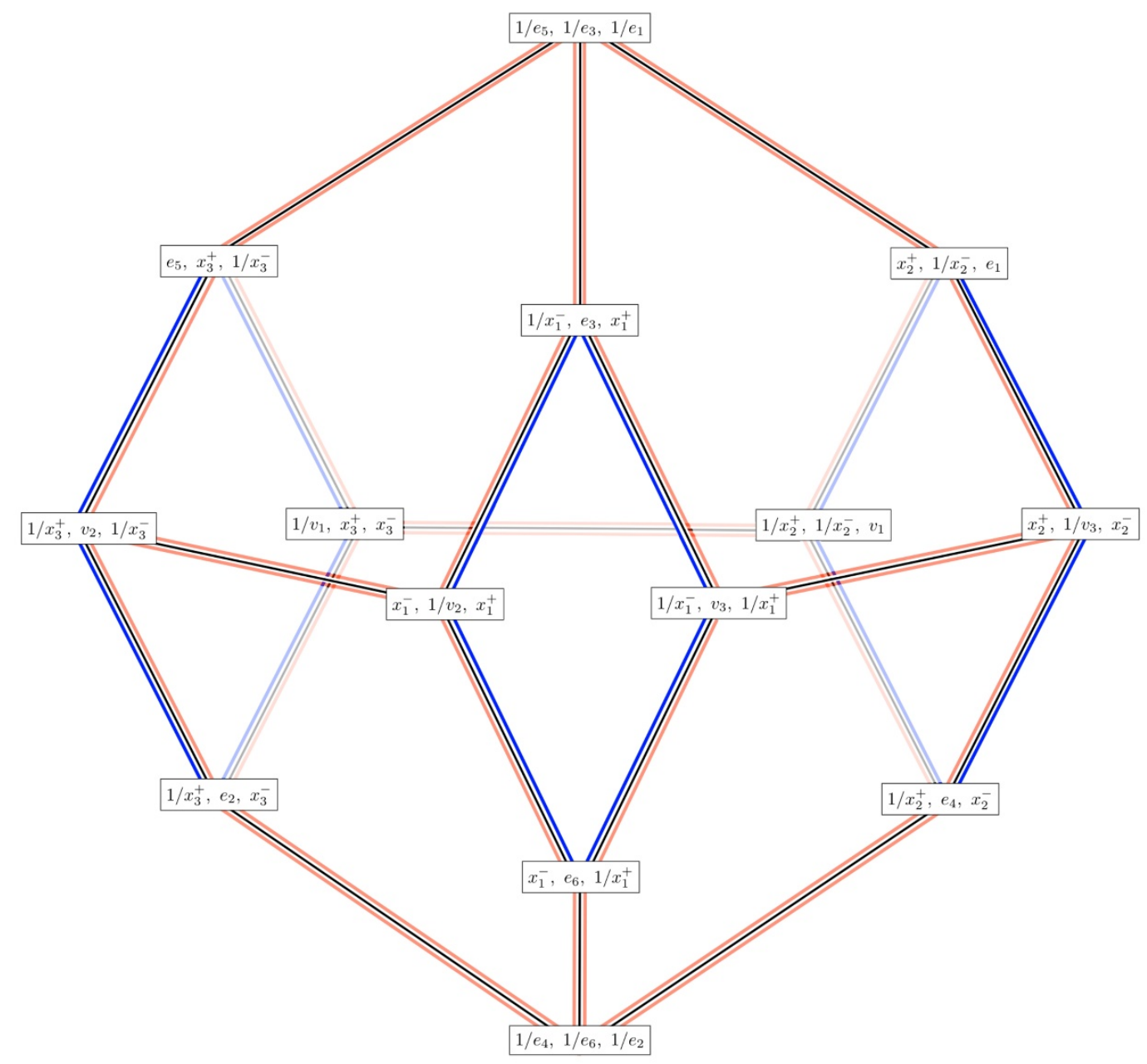

Figure 8. The Stasheff polytope for the $\operatorname{Gr}(2,6)$ (or $A_{3}$ ) cluster algebra, also known as the $K_{5}$ associahedron. Each of the 14 vertices (clusters) is labeled by its three $\mathcal{X}$-coordinates. The 21 edges connect pairs of clusters which are related to each other by some mutation. The 9 faces comprise three quadrilaterals (shown in blue) and six pentagons (shown in red). These correspond respectively to $A_{1} \times A_{1}$ and $A_{2}$ subalgebras.

These are the $\mathcal{X}$-coordinates of an $A_{2}$ cluster algebra. It can be checked that these are precisely minus the arguments of dilogarithms in the five-term dilogarithm identity (4.12). This is the dual of the statement shown in eq. (6.20).

The dual polytope, shown in figure 8, has 14 vertices and 9 faces, three of which are quadrilaterals and six of which are pentagons. This is the Stasheff polytope or the $K_{5}$ associahedron. The name associahedron comes from the following construction: consider $n$ (in the case of $K_{5}$ we take $n=5$ ) non-commutative variables and all the ways of inserting parentheses. For example, we have $((a b)(c d)) e,(((a b) c) d) e$, etc. In total there are $C_{n-1}$ ways of parenthesizing $n$ variables, where $C_{n}$ is the $n$th Catalan number. Then, join together two such expressions if one can be obtained from the other by applying the associativity rule once. By joining all these expressions, we build up the Stasheff polytope. 


\subsection{Cluster coordinates for $\operatorname{Gr}(3,7)$}

Beginning with the initial quiver for $\operatorname{Gr}(3,7)$, we can similarly generate all of the clusters and their $\mathcal{A}$ - and $\mathcal{X}$-coordinates by successive mutations until all possibilities are exhausted. The $E_{6}$ algebra generated in this manner has a total of 49 well-known $\mathcal{A}$-coordinates (see for example [57]), composed of the 35 Plücker coordinates $\langle i j k\rangle$ on $\operatorname{Gr}(3,7)$ together with 14 composite brackets of the form

$$
\langle 1 \times 2,3 \times 4,5 \times 6\rangle, \quad\langle 1 \times 2,3 \times 4,5 \times 7\rangle
$$

and their cyclic images. The seven coordinates $\langle 123\rangle, \ldots,\langle 712\rangle$ are frozen, while the remaining forty-two are unfrozen.

Mutation generates 833 distinct clusters, which altogether contain a total of 385 distinct $\mathcal{X}$-coordinates. We list all of them here by separating them into four classes, and use the notation

$$
r(a \mid b, c, d, e)=\frac{\langle a b c\rangle\langle a d e\rangle}{\langle a c d\rangle\langle a b e\rangle}
$$

as well as the $\mathbb{P}^{2}$ cross-ratio defined in eq. (4.9).

First of all there are $3 \times 7=21$ coordinates of the form

$$
r(2 \mid 1,3,5,6), \quad \frac{\langle 231\rangle\langle 456\rangle}{\langle 4 \times 5,6 \times 1,2 \times 3\rangle}, \quad \frac{\langle 127\rangle\langle 234\rangle\langle 345\rangle\langle 567\rangle}{\langle 257\rangle\langle 347\rangle\langle 1 \times 2,3 \times 4,5 \times 6\rangle}
$$

together with their cyclic images. Each of these cross-ratios is real (that is, equal to its parity conjugate - see appendix A), and it suffices to take only their cyclic images since a dihedral transformation (i.e., $i \rightarrow 8-i$ ) maps this set back to itself.

Secondly there are a further $2 \times 14=28$ real cross-ratios of the form

$$
r_{3}(1,2,5,6,3,4), \quad \frac{\langle 127\rangle\langle 256\rangle\langle 345\rangle}{\langle 257\rangle\langle 1 \times 2,3 \times 4,5 \times 6\rangle},
$$

together with their dihedral images.

Next come $6 \times 2 \times 7=84$ complex (that is, not equal to their parity conjugates) cross-ratios of the form

$$
\begin{array}{lll}
r(2 \mid 1,3,4,5), & r(1 \mid 6,3,4,5), & r(3 \mid 2,4,5,1), \\
r_{3}(1,5,3,2,6,4), & r_{3}(1,4,6,2,3,5), & \frac{\langle 127\rangle\langle 234\rangle\langle 567\rangle}{\langle 267\rangle\langle 3 \times 4,5 \times 7,1 \times 2\rangle},
\end{array}
$$

together with their parity conjugates and all cyclic images thereof.

Finally we have the $9 \times 2 \times 14=252$ complex cross-ratios

$$
\begin{aligned}
& r(1 \mid 5,2,3,4), \quad r(1 \mid 6,2,3,4), \quad r(1 \mid 6,2,3,5), \\
& r(1 \mid 6,2,4,5), \quad r(2 \mid 1,3,4,6), \\
& r_{3}(1,2,4,6,3,5), \quad r_{3}(1,4,3,6,5,2), \quad r_{3}(1,3,5,6,2,4), \quad \frac{\langle 261\rangle\langle 345\rangle}{\langle 4 \times 5,6 \times 1,2 \times 3\rangle},
\end{aligned}
$$

together with their parity conjugates and all dihedral images thereof. 
Note that we have expressed most of the cross-ratios above in a form in which they do not depend explicitly on point number 7 . The three most complicated cross-ratios are exceptions to this, and for these three we find it worthwhile to display here the non-trivial factorizations

$$
\begin{aligned}
1+\frac{\langle 127\rangle\langle 256\rangle\langle 345\rangle}{\langle 257\rangle\langle 1 \times 2,3 \times 4,5 \times 6\rangle} & =\frac{\langle 125\rangle\langle 7 \times 2,3 \times 4,5 \times 6\rangle}{\langle 257\rangle\langle 1 \times 2,3 \times 4,5 \times 6\rangle}, \\
1+\frac{\langle 127\rangle\langle 234\rangle\langle 567\rangle}{\langle 267\rangle\langle 3 \times 4,5 \times 7,1 \times 2\rangle} & =\frac{\langle 257\rangle\langle 1 \times 2,3 \times 4,6 \times 7\rangle}{\langle 267\rangle\langle 1 \times 2,3 \times 4,5 \times 7\rangle}, \\
1+\frac{\langle 127\rangle\langle 234\rangle\langle 345\rangle\langle 567\rangle}{\langle 257\rangle\langle 347\rangle\langle 1 \times 2,3 \times 4,5 \times 6\rangle} & =\frac{\langle 1 \times 2,3 \times 4,5 \times 7\rangle\langle 7 \times 2,3 \times 4,5 \times 6\rangle}{\langle 257\rangle\langle 347\rangle\langle 1 \times 2,3 \times 4,5 \times 6\rangle} .
\end{aligned}
$$

All three of the cluster $\mathcal{X}$-coordinates on the left-hand side of this equation appear in the $\mathrm{B}_{3} \otimes \mathbb{C}^{*}$ part of the coproduct of the two-loop $n=7 \mathrm{MHV}$ amplitude.

\subsection{Structure of the motivic two-loop $n=7$ MHV amplitude}

Obviously it is impractical for us to display the Stasheff polytope for the $\operatorname{Gr}(3,7)$ cluster algebra, with its 833 vertices, 2499 edges, and 2856 two-dimensional faces (of which 1785 are quadrilaterals and the other 1071 are pentagons). However, we are in a position now to carry out a 'motivic analysis' of the two-loop $n=7 \mathrm{MHV}$ amplitude using the information contained in the previous section.

First of all we note the amazing fact that all of the entries of $\{z\}_{2}$ and $\{z\}_{3}$ in the results in section 5 are always cluster $\mathcal{X}$-coordinates of $\operatorname{Conf}_{7}\left(\mathbb{P}^{2}\right)$. Interestingly, of the 385 such coordinates available, only 231 of them actually appear in the $n=7 \mathrm{MHV}$ amplitude at two loops. This might be a two-loop accident, but if it continues to hold at higher loop order it would be important to find some sort of geometric explanation.

Turning our attention now to the expression for the $\Lambda^{2} \mathrm{~B}_{2}$ part of the coproduct shown in eq. (5.2), we note first of all the further highly nontrivial fact that for each term $\left\{x_{1}\right\}_{2} \wedge$ $\left\{x_{2}\right\}_{2}$, there is always at least one of the 833 clusters which contains both $x_{1}$ and $x_{2}$. And more spectacularly, the variables always appear in pairs with Poisson bracket $\left\{x_{1}, x_{2}\right\}=0$. Now we understand the geometric meaning of the ambiguity mentioned in eq. (5.4), in light of eq. (6.19) - it is exactly the ambiguity of trying to chose one of the four vertices of a quadrilateral, when there is no reason at all to have to make a choice: each term in $\Lambda^{2} \mathrm{~B}_{2}$ corresponds naturally to a certain quadrilateral face.

We conclude that the most canonical, invariant way of expressing the $\Lambda^{2} \mathrm{~B}_{2}$ part of the coproduct of the two-loop $n=7 \mathrm{MHV}$ amplitude is not by the formula (5.2), but by writing it as a sum of 42 quadrilateral faces of the $E_{6}$ Stasheff polytope. It is obviously of paramount importance to understand what makes these 42 special, out of the 1785 such faces available.

An analysis of the $\mathrm{B}_{3} \otimes \mathbb{C}^{*}$ part of the coproduct requires a classification of all of the possible $A_{3}, A_{2} \times A_{1}$ and $A_{1} \times A_{1} \times A_{1}$ subalgebras of $E_{6}$. The generalized Stasheff polytope of this algebra has 1547 three-dimensional faces, consisting of 357 cubes $\left(A_{1} \times A_{1} \times A_{1}\right), 714$ pentaprisms $\left(A_{2} \times A_{1}\right)$ and 476 of the $A_{3}$ polytopes shown in figure 8 . We expect these to play a role in unlocking further structure in the two-loop $n$-point MHV amplitudes, which we will explore in future work. 


\section{Conclusion}

Appropriately defined scattering amplitudes in maximally supersymmetric Yang-Mills theory are functions on $\operatorname{Conf}_{n}\left(\mathbb{P}^{3}\right)$ which have a very rich mathematical structure but do not, in general, appear to admit any particular canonical or even preferred functional representation. The one important exception is the two-loop MHV amplitude for $n=6$ reviewed in section (3), which does have a canonical form (up to trivial $\mathrm{Li}_{m}$ identities): that in which it is expressed only in terms of the classical polylogarithm functions, with only (minus) cluster $\mathcal{X}$-coordinates as arguments.

More general amplitudes may be computed numerically if desired (for example all two-loop MHV amplitudes may be evaluated with reasonable efficiency [61]), but we do not strive to find any particular explicit analytic formulas for them. It often happens in physics and in mathematics that when one reaches sufficiently deep into a subject, one realizes that the appropriate objects of study are not what one originally thought, but some suitable generalization or modification thereof. In this vein we have proposed that our focus on the mathematical structure of scattering amplitudes in SYM theory should fall on what we call motivic amplitudes, and in particular on their coproducts, which capture the 'mathematically most complicated part' of an amplitude.

By drawing on our explicit results for the two-loop $n=6$ and $n=7$ MHV motivic amplitudes, we have shown that an important role is played by cluster coordinates, which are preferred sets of coordinates on $\operatorname{Conf}_{n}\left(\mathbb{P}^{3}\right)$ with very rich mathematical structure. Specifically, we conjecture based on the examples presented here, as well as others that we have computed, that all coproduct components of all two-loop MHV motivic amplitudes are expressible in terms of Bloch group elements $\{x\}_{k}$ with only $\mathcal{X}$-coordinates $x$ appearing. The algebras relevant for $n=6,7$ are of finite type, being the $A_{3}$ and $E_{6}$ algebras respectively, while for $n>7$ the relevant algebras are of infinite type, although only a finite subset of these variables actually appear at two loops.

If one accepts that cluster $\mathcal{X}$-coordinates answer the 'kinematical' question which variables do motivic amplitudes depend on?, it is natural to turn attention next to the 'dynamical' question of exactly in which combinations they appear in amplitudes. We have provided a first glimpse by showing that the terms in $\Lambda^{2} \mathrm{~B}_{2}$ component of the coproduct of the two-loop $n=7 \mathrm{MHV}$ amplitude - the component which measures the obstruction to writing this amplitude in terms of the classical polylogarithm functions only are in correspondence with quadrilateral faces of the relevant Stasheff polytope (i.e., with $A_{1} \times A_{1}$ subalgebras of the cluster algebra). Again based on other examples which we have analyzed, we conjecture that this statement remains true for all two-loop MHV amplitudes. However a great deal of structure remains to be understood. In particular, only a very small number of all possible quadrilaterals actually make an appearance in $\Lambda^{2} \mathrm{~B}_{2}$; what, if anything, is the special geometric significance of these particular quadrilaterals? What is the geometric significance of the cluster $\mathcal{X}$-coordinates appearing in $\mathrm{B}_{3} \otimes \mathbb{C}^{*}$, or in non-MHV amplitudes, or at higher loops? A few of these questions will be addressed in future work.

Many other interesting questions are also raised by our work. For example, Dixon, Drummond and Henn have employed with great success the strategy of studying the space 
of all integrable, conformally invariant symbols whose letters are drawn from the collection of available $\mathcal{A}$-coordinates at $n=6$. By imposing all physical constraints available to them at the time, they were able to determine the symbol of the two-loop NMHV amplitude exactly [62], and that of the three-loop MHV amplitude up to two parameters [63] which were subsequently determined in [64]. We have proposed that only $\mathcal{X}$-coordinates can appear in the coproduct of MHV amplitudes, which is a stronger condition than that only $\mathcal{A}$-coordinates can appear in their symbols. The functions $\operatorname{Li}_{m}(1+x)$ and $\operatorname{Li}_{m}(1+1 / x)$ for any $\mathcal{X}$-coordinate $x$ for example satisfy the latter but not the former. Hence in particular we expect to see neither $\operatorname{Li}_{3}\left(u_{i}\right)$ nor $\operatorname{Li}_{3}\left(1-u_{i}\right)$ in the coproduct of any two-loop MHV motivic amplitudes. It would be very interesting to investigate in detail how restrictive this condition is in the space of all integrable symbols, in order to see whether our new 'motivic' constraint could aid future computations employing this strategy.

It is also important to point out that in the examples we've looked at, only a fraction of all available $\mathcal{X}$-coordinates actually make an appearance. For example in section 3 we emphasized that only the 9 coordinates (3.4) enter the two-loop $n=6$ amplitude, out of the 15 available. Then in section 5 we found that only 231 of the 385 available $\mathcal{X}$-coordinates make an appearance in the two-loop $n=7$ motivic amplitude. We do not yet have an understanding of the criterion which selects these particular subsets of $\mathcal{X}$-coordinates, nor whether this phenomenon is just an accident at two loops or continues to hold at higher loop order. If it does, this obviously constrains the space of possible motivic amplitudes even more strongly than just the $\mathcal{X}$-coordinate condition discussed in the previous paragraph. We cannot help but note with amusement the fact that $9 / 15=231 / 385$, but we certainly have too little data to speculate on whether or not this is just a coincidence.

A number of interesting questions about the connection between motivic amplitudes, cluster coordinates and other recent approaches to scattering amplitudes can now be asked. A fair amount of recent work has considered the behavior of amplitudes in various restricted domains, such as two-dimensional or multi-Regge kinematics (see for example [6569] or [70-75], respectively), where in either case considerable simplification occurs. Also, it has long been appreciated that the behavior of amplitudes under collinear (and especially multi-collinear) limits strongly constrains their structure, and the operator product expansion (OPE) to the null polygonal Wilson loop [76-79] aims to compute arbitrary amplitudes at finite coupling in a systematic expansion around the collinear limit. It would very nice to have a thorough understanding of these limits and expansions directly at the level of cluster algebras, or even individual quivers.

Finally, we have so far made no explicit reference to the integrability of planar SYM theory (see the review [80]), which clearly plays a crucial but so far not fully exploited part in unlocking the structure of its amplitudes (approaches other than the OPE mentioned above include for example [81-84]). We hope that motivic amplitudes and cluster coordinates will be found to be useful in these and other endeavors, just as general 'motivic' methods based on the symbol calculus of polylogarithm functions are finding ever wider applications to physical computations in quantum field theory, including Feynman integrals, amplitudes, form factors, correlation functions, and Wilson loops [85-92], not just in SYM theory but even QCD [93-97] and string theory [10-13] as well. 


\section{Acknowledgments}

Various subsets of us have benefited from stimulating discussions with Nima Arkani-Hamed, Lance Dixon, James Drummond and David Skinner, and are grateful to Andy Neitzke and Yang-Hui He for very illuminating conversations on cluster algebras. MS, CV and AV appreciate the generous support of the Kavli Institute for Theoretical Physics during the initial stages of this work, and CV in addition acknowledges the hospitality of BIRS, ECT* and CERN during its course. This work was supported by the US Department of Energy under contracts DE-FG02-91ER40688 (JG, MS) and DE-FG02-11ER41742 (AV Early Career Award), the Simons Fellowship in Theoretical Physics (AV), and the Sloan Research Foundation (AV). The work of AG is supported by the NSF grants DMS-1059129 and DMS-1301776.

\section{A Parity conjugation on $\operatorname{Conf}_{n}\left(\mathbb{P}^{k-1}\right)$}

\section{A.1 Positive configurations}

Given a volume form $\omega$ in $V_{k}$, which is not a part of our data, we can assign to a configuration of $k$ vectors $v_{1}, \ldots, v_{k}$ a number:

$$
\langle 1, \ldots, k\rangle:=\left\langle v_{1}, \ldots, v_{k}\right\rangle:=\omega\left(v_{1}, \ldots, v_{k}\right) .
$$

Given an orientation of a real vector space $V_{k}$, we can define positive configurations of vectors in $V_{k}$. Namely, choose a volume form $\omega$ compatible with the orientation of the space, i.e. $\omega\left(v_{1}, \ldots, v_{k}\right)>0$ if $\left(v_{1}, \ldots, v_{k}\right)$ is a positively oriented basis. Then a configuration $\left(v_{1}, \ldots, v_{n}\right)$ is positive if $\left\langle v_{i_{1}}, \ldots, v_{i_{k}}\right\rangle>0$ for any $i_{1}<\cdots<i_{k}$. We denote by $\operatorname{Conf}_{n}^{+}(k)$ the set of positive configurations.

There is a twisted cyclic shift map, obtained by moving the last vector to the front, and multiplying it by $(-1)^{k-1}$. It preserves positive configurations of vectors:

$$
c: \operatorname{Conf}_{n}^{+}(k) \longrightarrow \operatorname{Conf}_{n}^{+}(k), \quad\left(v_{1}, \ldots, v_{n}\right) \longmapsto\left((-1)^{k-1} v_{n}, v_{1}, \ldots, v_{n-1}\right) .
$$

The subspace of positive configurations of points $\operatorname{Conf}_{n}^{+}\left(\mathbb{R P}^{k-1}\right)$ is the image of the restriction of the projection map $\pi: \operatorname{Conf}_{n}(k) \longrightarrow \operatorname{Conf}_{n}\left(\mathbb{P}^{k-1}\right)$ to positive configurations of vectors:

$$
\operatorname{Conf}_{n}^{+}\left(\mathbb{R P}^{k-1}\right):=\pi\left(\operatorname{Conf}_{n}^{+}(k)\right)
$$

\section{A.2 Parity conjugation}

Let $P_{n}$ be an oriented convex $n$-gon. Let us denote by $\operatorname{Conf}_{P_{n}}\left(\mathbb{R} \mathbb{P}^{k-1}\right)$ the space of configurations of points of $\mathbb{P}^{k-1}$ parametrized by the set of vertices of the polygon $P_{n}$. It is the space of orbits of the diagonal action of the group $P G L_{k}$ on collections of points parametrized by the vertices.

We emphasize that the points are parametrized by the vertices of the polygon, but there is no special parametrization of the vertices. If we choose an initial vertex $v$ of the polygon, then there is an isomorphism

$$
i_{v}: \operatorname{Conf}_{P_{n}}\left(\mathbb{R} \mathbb{P}^{k-1}\right) \longrightarrow \operatorname{Conf}_{n}\left(\mathbb{P}^{k-1}\right)
$$


defined by parameterizing the vertices by the set $\{1, \ldots, n\}$, starting from the vertex $v$ to which we assign 1, and going according to the orientation of the polygon.

The parity conjugation is a rational map, i.e. a map defined for generic configurations,

$$
*: \operatorname{Conf}_{P_{n}}\left(\mathbb{R P}^{k-1}\right) \longrightarrow \operatorname{Conf}_{* P_{n}}\left(\mathbb{R P}^{k-1}\right),
$$

described as follows. A collection of points $\left\{x_{v}\right\}$ in $\mathbb{P}^{k-1}$ parametrized by the set of vertices $\{v\}$ of the polygon $P_{n}$ gives rise to a collection of hyperplanes $\left\{H_{v}\right\}$ in $\mathbb{P}^{k-1}$ parametrized by the same set. Namely, let $\left\{x_{1}(v), \ldots, x_{k-1}(v)\right\}$ be the points parametrized by the $(k-1)$ vertices of the polygon obtained by starting at the vertex $v$ and going around the polygon following the orientation. So $x_{1}(v)=x_{v}$ and so on. We define the hyperplane $H_{v}$ to be the span of these points:

$$
H_{v}:=\left\langle x_{1}(v), \ldots, x_{k-1}(v)\right\rangle .
$$

Viewing the hyperplanes $\left\{H_{v}\right\}$ as points of the dual projective space, and reversing the orientation of the polygon, we get a point of $\operatorname{Conf}_{* P_{n}}\left(\mathbb{R} \mathbb{P}^{k-1}\right)$, which is the result of the parity conjugation applied to the original configuration of points.

Lemma A.1. The map (A.5) is a perfect duality: $*^{2}=$ Id.

Proof. To calculate the map $*^{2}$ we need to find the intersection of the hyperplanes $H_{w}$ corresponding to the vertex $v$ and $k-2$ vertices preceding it in the orientation of $P_{n}$. All of them, by the very definition, contain the point $x_{v}$ parametrized by the vertex $v$.

We emphasize that the map (A.5) is not a map of a space to itself, since there is no invariant way to identify the left and right spaces in (A.5).

\section{A.3 Parity conjugation on configurations of vectors}

Similarly, let us denote by $\operatorname{Conf}_{P_{n}}(k)$ the space of configurations of vectors in a $k$ dimensional vector space, parametrized by the vertices of the polygon $P_{n}$. Let us upgrade the projective parity conjugation to a parity conjugation on configurations vectors, given by a rational map

$$
*: \operatorname{Conf}_{P_{n}}(k) \longrightarrow \operatorname{Conf}_{* P_{n}}(k) .
$$

Having in mind computing the parity conjugation, we define it now by breaking the symmetry, i.e. using the isomorphism $i_{v}: \operatorname{Conf}_{P_{n}}(k) \rightarrow \operatorname{Conf}_{n}(k)$ determined by a choice of a specific vertex $v$. So we use the polygon vertex $v$ to order the vectors of a configuration by $\left(l_{1}, \ldots, l_{n}\right)$. Let us define a configuration of covectors $\left(g_{1}, \ldots, g_{n}\right)$ by setting

$$
g_{1}(\bullet):=\frac{\omega\left(l_{1}, \ldots, l_{k-1}, \bullet\right)}{\omega\left(l_{1}, \ldots, l_{k-1}, l_{k}\right)},
$$

and $g_{i}$ obtained by the twisted cyclic shift by $i-1$ of this formula. The covectors $g_{i}$ evidently do not depend on the choice of the form $\omega$.



$$
*: \operatorname{Conf}_{n}(k) \longrightarrow \operatorname{Conf}_{n}(k), \quad *\left(l_{1}, \ldots, l_{n}\right):=\left(g_{1}, \ldots, g_{n}\right) \text {. }
$$


Abusing notation, we use the same notation $*$. Let us stress again that, although $g_{i}$ 's are vectors of the dual space, the configurations of vectors in a space and in its dual are canonically identified.

Proposition A.2. The map

$$
*:\left(l_{1}, \ldots, l_{n}\right) \longmapsto\left(g_{1}, \ldots, g_{n}\right)
$$

is a duality.

For computational purposes, we define a non-normalized version of the parity map

$$
f_{1}(\bullet):=\omega\left(l_{1}, \ldots, l_{k-1}, \bullet\right)
$$

and $f_{i}$ is obtained by the twisted cyclic shift by $i-1$.

A volume form $\omega$ in $V_{k}$ defines the dual volume form $* \omega$ on $V_{k}^{*}$. We set

$$
[1, \ldots, k]:=\left[f_{1}, \ldots, f_{k}\right]:=* \omega\left(f_{1}, \ldots, f_{k}\right)
$$

Lemma A.3. One has

$$
\left[f_{1}, f_{2}, \ldots, f_{k}\right]=\langle 1,2, \ldots, k\rangle\langle 2,3, \ldots, k+1\rangle \ldots\langle k-1, k, \ldots, 2 k-2\rangle .
$$

For example, for $k=4$ we have

$$
\left[f_{1}, f_{2}, f_{3}, f_{4}\right]=\langle 1,2,3,4\rangle\langle 2,3,4,5\rangle\langle 3,4,5,6\rangle \text {. }
$$

Proof. The left-hand side in (A.13) vanishes if any of the brackets on the right vanishes. For example, if $\langle 1,2, \ldots, k\rangle=0$, then $f_{1}$ and $f_{2}$ are proportional, and thus $\left[f_{1}, \ldots, f_{k}\right]=0$. Therefore the left hand side is divisible by their product. Since they are polynomials of the same degree, the claim follows up to a constant. The constant must be \pm 1 : indeed, it is a rational number; if it is divisible by a prime $p$, reducing $\bmod p$ we get a contradiction. Notice that the two expressions in (A.13) scale the same way under the rescaling $v_{i} \longmapsto \lambda_{i} v_{i}$. Rescaling $\omega \longmapsto \lambda \omega$ we rescale $f_{*}$ by $\lambda$ and $[*]$ by $\lambda^{-1}$. Thus both sides scale by $\lambda^{k-1}$.

Proof of the Proposition. The map $*^{2}$, being projected to configurations of points, is the identity map. Here it is crucial that the change of the orientation of the polygon $P_{n}$ is built in its definition. Lemma A.3 tells

$$
\left[g_{1}, g_{2}, \ldots, g_{k}\right]=\langle k, \ldots, 2 k-1\rangle^{-1}
$$

Therefore $\left\langle * g_{1}, \ldots, * g_{k}\right\rangle=\langle 1, \ldots, k\rangle$. This implies the proof for odd $n$, and thus, by employing a trick, for all $n$. 

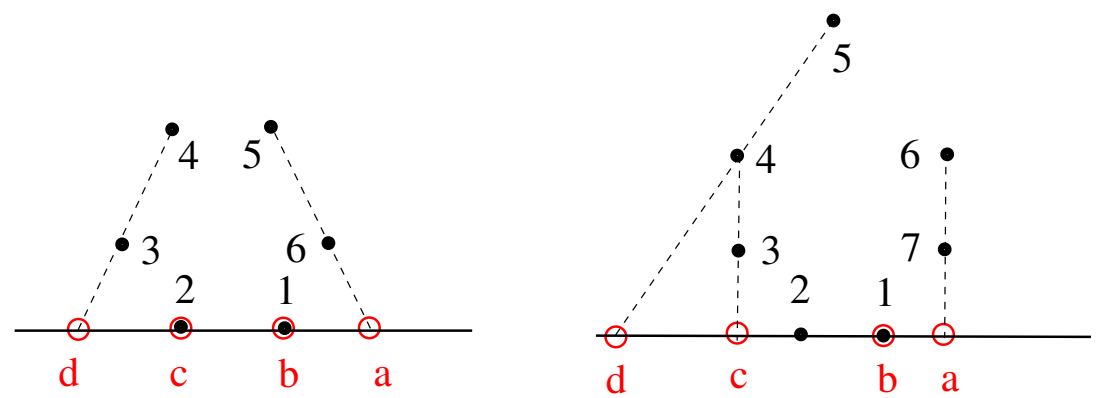

Figure 9. $r(a, b, c, d)=\frac{\langle 234\rangle\langle 156\rangle}{\langle 1 \times 2,3 \times 4,5 \times 6\rangle}$ (left), and $r(a, b, c, d)=\frac{\langle 123\rangle\langle 345\rangle\langle 671\rangle}{\langle 134\rangle\langle 1 \times 2,4 \times 5,6 \times 7\rangle}$ (right).

\section{A.4 Parity conjugation for the projective plane}

It is convenient to use the notation $f_{i j}$ for the functional defined as $f_{i j}(v):=\omega\left(v_{i}, v_{j}, v\right)$. One has

$$
\begin{aligned}
& {\left[f_{12}, f_{23}, f_{34}\right]=\langle 1,2,3\rangle\langle 2,3,4\rangle .} \\
& {\left[f_{12}, f_{23}, f_{45}\right]=\langle 1,2,3\rangle\langle 2,4,5\rangle .}
\end{aligned}
$$

To prove the second identity, notice that if any of the two factors becomes zero, then the left hand side is zero. For example, if $v_{5}$ is a linear combination of $v_{2}$ and $v_{4}$, then the left hand side is proportional to $\left[f_{12}, f_{23}, f_{24}\right]=0$.

Using this, we easily calculate some examples of the parity involution action for configurations of points in $\mathbb{P}^{2}$ :

$$
\begin{aligned}
r(1 \mid 2,3,4,5) & =\frac{\langle 123\rangle\langle 145\rangle}{\langle 134\rangle\langle 125\rangle} \longmapsto r_{3}(2,5,4 ; 1,6,3)=-\frac{\langle 251\rangle\langle 546\rangle\langle 423\rangle}{\langle 256\rangle\langle 543\rangle\langle 421\rangle}, \\
r(1 \mid 2,3,5,7) & =\frac{\langle 123\rangle\langle 157\rangle}{\langle 135\rangle\langle 127\rangle} \longmapsto \frac{\langle 234\rangle\langle 156\rangle}{\langle 1 \times 2,3 \times 4,5 \times 6\rangle}, \\
r(1 \mid 3,4,6,7) & =\frac{\langle 134\rangle\langle 167\rangle}{\langle 146\rangle\langle 137\rangle} \longmapsto \frac{\langle 124\rangle\langle 345\rangle\langle 671\rangle}{\langle 134\rangle\langle 1 \times 2,4 \times 5,6 \times 7\rangle}, \\
r(1 \mid 3,4,5,6) & =\frac{\langle 134\rangle\langle 156\rangle}{\langle 145\rangle\langle 136\rangle} \longmapsto \frac{\langle 345\rangle\langle 124\rangle\langle 567\rangle\langle 126\rangle}{\langle 456\rangle\langle 125\rangle\langle 1 \times 2,3 \times 4,6 \times 7\rangle}, \\
r_{3}(1,2,4 ; 7,3,6) & =-\frac{\langle 127\rangle\langle 243\rangle\langle 416\rangle}{\langle 123\rangle\langle 246\rangle\langle 417\rangle} \longmapsto \frac{\langle 345\rangle\langle 1 \times 2,4 \times 5,6 \times 7\rangle}{\langle 145\rangle\langle 2 \times 3,4 \times 5,6 \times 7\rangle} .
\end{aligned}
$$

Let us give two examples of the proofs of these formulas.

1. The formula illustrated in figure 10 was proved in [53]. Here is a different proof:

$$
\begin{aligned}
\left(f_{14} \mid f_{23}, f_{25}, f_{61}, f_{36}\right) & =\frac{\left[f_{14}, f_{23}, f_{25}\right]\left[f_{14}, f_{61}, f_{36}\right]}{\left[f_{14}, f_{25}, f_{61}\right]\left[f_{14}, f_{23}, f_{36}\right]} \stackrel{(A .16),(A .17)}{=} \\
\frac{\langle 325\rangle\langle 214\rangle\langle 361\rangle\langle 614\rangle}{\langle 614\rangle\langle 125\rangle\langle 236\rangle\langle 314\rangle} & =-\frac{\langle 124\rangle\langle 235\rangle\langle 316\rangle}{\langle 125\rangle\langle 236\rangle\langle 314\rangle}
\end{aligned}
$$

The cross-ratio $r\left(f_{14} \mid f_{23}, f_{25}, f_{61}, f_{36}\right)$ can be calculated by viewing the points $f_{i j}$ of the dual projective plane as the lines, denoted by $L_{i j}$, in the original projective plane. 


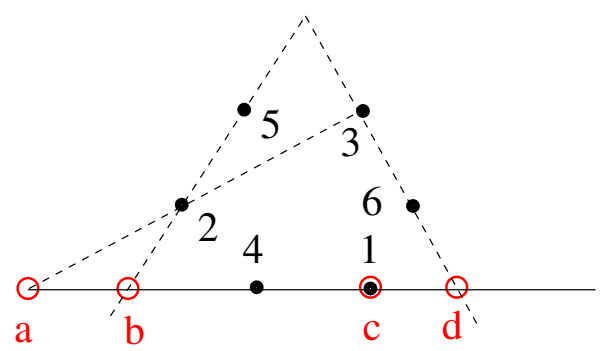

Figure 10. $r(a, b, c, d)=r(1 \mid 2,4,3,25 \cap 36)=r_{3}(1,2,3 ; 4,5,6)=-\frac{\langle 124\rangle\langle 235\rangle\langle 316\rangle}{\langle 125\rangle\langle 236\rangle\langle 314\rangle}$.



Figure 11. $r(a, b, c, d)=\frac{(345)(124)(567)(126)}{(456)(125)(1 \times 2,3 \times 4,6 \times 7)}$.

Then it is the cross-ratio of the configuration of four points obtained by intersecting the line $L_{14}$ with the lines $L_{23}, L_{25}, L_{61}, L_{36}$. This configuration is nothing else but the configuration of points $(a, b, c, d)$ on the line $L_{14}$. So we arrive at the geometric interpretation of the triple ratio as the cross-ratio [54] given on the left of figure 10:

$$
r(1 \mid 2,4,3,25 \cap 36)=r_{3}(1,2,3 ; 4,5,6)=-\frac{\langle 124\rangle\langle 235\rangle\langle 316\rangle}{\langle 125\rangle\langle 236\rangle\langle 314\rangle} .
$$

Notice that $r(2 \mid 3,5,1,14 \cap 36)=r(1 \mid 2,4,3,25 \cap 36)$; to see this, project onto the 36 line.

2. To check formula (A.19) we write

$$
\begin{aligned}
& r\left(f_{12} \mid f_{23}, f_{34}, f_{56}, f_{71}\right)=\frac{\left[f_{12}, f_{23}, f_{34}\right]\left[f_{12}, f_{56}, f_{71}\right]}{\left[f_{12}, f_{34}, f_{56}\right]\left[f_{12}, f_{23}, f_{71}\right]} \stackrel{(A .16),(A .17)}{=} \\
& \frac{\langle 123\rangle\langle 234\rangle\langle 712\rangle\langle 156\rangle}{\left[f_{12}, f_{34}, f_{56}\right]\langle 123\rangle\langle 271\rangle}=\frac{\langle 156\rangle\langle 234\rangle}{\langle 1 \times 2,3 \times 4,5 \times 6\rangle}
\end{aligned}
$$

This provides the geometric interpretation of the cluster $\mathcal{X}$-coordinate (A.19) as the cross-ratio given on the left of figure 9 .

Therefore all of the more complicated cluster $\mathcal{X}$-coordinates for $\operatorname{Conf}_{7}\left(\mathbb{P}^{3}\right)$ can be obtained by applying the parity involution to the standard cross-ratios $r$ and the triple ratios $r_{3}$. 

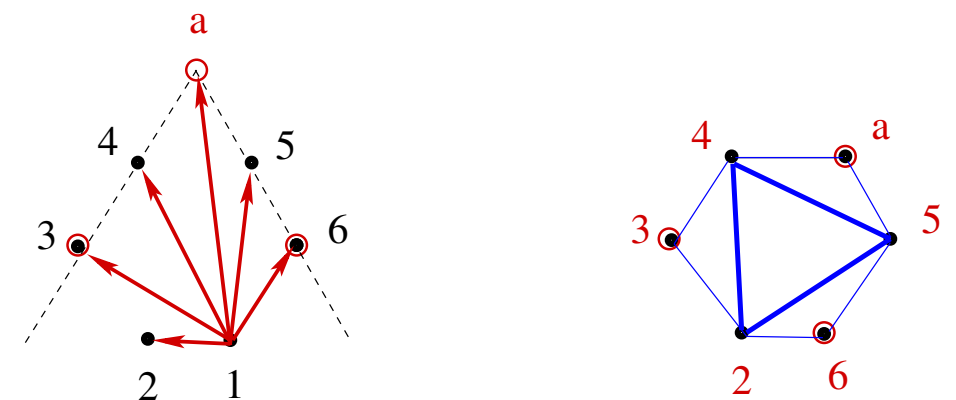

Figure 12. Geometric meaning of the first three terms in (B.1), given by cross-ratios (B.2).

\section{B An identity for the trilogarithm in cluster $\mathcal{X}$-coordinates}

As we emphasized in section 4, Abel's pentagon equation for the dilogarithm admits a form where the set of arguments is the set of all (negated) cluster $\mathcal{X}$-coordinates on the space of configurations of five points in $\mathbb{P}^{1}$ : this is the example of the $A_{2}$ algebra reviewed in section 6 , though the properties of the pentagram of arguments were studied already by Gauss [98].

It is natural to ask whether there is a natural generalization of this feature of Abel's identity to higher polylogarithms. There is a generic functional equation for the trilogarithm related to the space of configurations of seven points in $\mathbb{P}^{2}$, see $[53,54]$, from which any functional equation for the trilogarithm can be deduced. Its arguments are the triple ratios, which, as we now know, are the simplest examples of cluster $\mathcal{X}$-coordinates which go beyond the $\mathbb{P}^{1}$ cross-ratios. However, the collection of all its arguments is invariant under the action of the permutation group $S_{7}$, and so cannot be a subset of the set of cluster coordinates. Instead, we have found the following

Theorem B.1. Given a configuration of six points in $\mathbb{P}^{2}$, there is a 40-term functional equation for the classical trilogarithm:

$$
\begin{aligned}
&\{(1 \mid 2,3,4,5)\}_{3}+\{(1 \mid 2,4,5,6)\}_{3}+\left\{r_{3}(1,4,5 ; 2,3,6)\right\}_{3} \\
&+ \frac{1}{3}\left\{r_{3}(1,3,5 ; 6,2,4)\right\}_{3}+\text { signed dihedral permutations }=0
\end{aligned}
$$

in $\mathrm{B}_{3}(\mathbb{C})$. Here the six cyclic permutations are taken with plus sign, and the six anticyclic permutations are with minus sign.

The remarkable feature of the functional equation (B.1) for the trilogarithm is that each of its 40 arguments is a cluster $\mathcal{X}$-coordinate on the space of configurations of six points in $\mathbb{P}^{2}$. This is the first known functional equation for the trilogarithm with the property that all arguments are cluster $\mathcal{X}$-coordinates of the same algebra.

Here is a geometric interpretation of the functional equation (B.1). Take a configuration of six points $\left(x_{1}, \ldots, x_{6}\right)$ in $\mathbb{P}^{2}$. Let $a:=x_{3} x_{4} \cap x_{5} x_{6}$ be the intersection point of the lines $x_{3} x_{4}$ and $x_{5} x_{6}$. The lines passing through $x_{1}$ form a projective line. The points $x_{2}, x_{3}, x_{4}, a, x_{5}, x_{6}$ determine a configuration of six point on that line, denoted by 
$\left(x_{1} \mid x_{2}, x_{3}, x_{4}, a, x_{5}, x_{6}\right)$, and illustrated on the left in figure 12 . The three cross-ratios

$$
r\left(x_{1} \mid x_{2}, x_{3}, x_{4}, x_{5}\right), \quad r\left(x_{1} \mid x_{2}, x_{4}, x_{5}, x_{6}\right), \quad r\left(x_{1} \mid x_{4}, a, x_{5}, x_{2}\right)
$$

are the arguments of the first three terms in (B.1), up to the inversion of the third one, which does not affect the corresponding element of the group $B_{3}$. Indeed, the identity (A.24) shows that

$$
r\left(x_{1} \mid x_{4}, a, x_{5}, x_{2}\right)=r\left(x_{1} \mid x_{4}, x_{2}, x_{5}, a\right)^{-1}=r_{3}(1,4,5 ; 2,3,6)^{-1} .
$$

The three cross-ratios (B.2) are the cluster $\mathcal{X}$-coordinates on the space of configurations $\left(x_{1} \mid x_{2}, x_{3}, x_{4}, a, x_{5}, x_{6}\right)$ of six points on $\mathbb{P}^{1}$ corresponding to the triangulation of the hexagon in figure 12. They are assigned to the three diagonals of the triangulation; the points of the configuration $\left(x_{1} \mid x_{2}, x_{3}, x_{4}, a, x_{5}, x_{6}\right)$ are situated at the vertices of the hexagon.

Proof of Theorem B.1. We need to check that applying the cobracket $\delta$ to the 40-term expression (B.1) we get zero in $\mathrm{B}_{2}(\mathbb{C}) \otimes \mathbb{C}^{*}$. Due to the (signed) dihedral symmetry, it is sufficient to calculate the expressions $\mathrm{B}_{2}^{\langle 123\rangle} \otimes\langle 123\rangle, \mathrm{B}_{2}^{\langle 124\rangle} \otimes\langle 124\rangle$ and $\mathrm{B}_{2}^{\langle 135\rangle} \otimes\langle 135\rangle$ in $\mathbb{Z}[\mathbb{C}] \otimes \mathbb{C}^{*}$. One has $\mathrm{B}_{2}^{\langle 135\rangle}=0$. The other two we present as sums of the five-term relations of geometric origin. Before we formulate the answer, let us recall that

$$
\begin{aligned}
& \frac{\langle 123\rangle\langle 456\rangle}{\langle 1 \times 2,3 \times 4,5 \times 6\rangle}=r(1 \mid 2,3,4,34 \cap 56), \\
& \frac{\langle 1 \times 2,3 \times 4,5 \times 6\rangle}{\langle 156\rangle\langle 234\rangle}=r(5 \mid 1,2,12 \cap 34,6) .
\end{aligned}
$$

Using this, one can calculate that

$$
\begin{aligned}
\mathrm{B}_{2}^{(123)}= & -\left(\{r(5 \mid 6,1,3,4)\}_{2}+\left\{r_{3}(1,5,4 ; 2,6,3)\right\}_{2}+\left\{r_{3}(1,3,5 ; 2,4,6)\right\}_{2}\right. \\
& \left.+\{r(1 \mid 5,2,3,4)\}_{2}+\{r(1 \mid 2,3,4,34 \cap 56)\}_{2}\right) \\
- & \left(\{r(3 \mid 2,4,6,1)\}_{2}+\left\{r_{3}(2,3,6 ; 1,4,5)\right\}_{2}+\left\{r_{3}(1,6,3 ; 2,5,4)\right\}_{2}\right. \\
& \left.+\{r(6 \mid 5,1,2,3)\}_{2}+\{r(5 \mid 1,2,12 \cap 34,6)\}_{2}\right) \\
& +\left(\{r(4 \mid 5,1,2,3)\}_{2}+\left\{r_{3}(2,4,5 ; 1,3,6)\right\}_{2}+\left\{r_{3}(1,5,4 ; 2,6,3)\right\}_{2}\right. \\
& \left.+\{r(5 \mid 6,1,2,4)\}_{2}+\{r(5 \mid 1,2,12 \cap 34,6)\}_{2}\right) \\
& -\left(\{r(5 \mid 2,3,4,6)\}_{2}+\left\{r_{3}(2,4,5 ; 1,3,6)\right\}_{2}+\left\{r_{3}(2,5,3 ; 1,6,4)\right\}_{2}\right. \\
& \left.+\{r(2 \mid 1,3,4,5)\}_{2}+\{r(2 \mid 1,34 \cap 56,4,3)\}_{2}\right) \\
& - \text { permutation by }(321654) .
\end{aligned}
$$


Similarly,

$$
\begin{aligned}
\mathrm{B}_{2}^{(124)}=\left(\{r(1 \mid 2,4,5,6)\}_{2}+\{r(2 \mid 4,5,6,1)\}_{2}+\{r(4 \mid 5,6,1,2)\}_{2}\right. \\
\left.+\{r(5 \mid 6,1,2,4)\}_{2}+\{r(6 \mid 1,2,4,5)\}_{2}\right) \\
-\left(\{r(5 \mid 1,6,12 \cap 34,2)\}_{2}+\{r(4 \mid 1,2,3,6)\}_{2}+\left\{r_{3}(2,6,4 ; 1,5,3)\right\}_{2}\right. \\
\left.\quad+\left\{r_{3}(1,4,6 ; 2,3,5)\right\}_{2}+\{r(6 \mid 1,2,4,5)\}_{2}\right) \\
-\left(\{r(5 \mid 1,2,12 \cap 34,6)\}_{2}+\{r(4 \mid 2,3,5,1)\}_{2}+\left\{r_{3}(2,4,5 ; 1,3,6)\right\}_{2}\right. \\
\left.+\left\{r_{3}(1,5,4 ; 2,6,3)\right\}_{2}+\{r(5 \mid 2,4,6,1)\}_{2}\right) .
\end{aligned}
$$

To present each of the seven five-term summands as a five term relation, define

$$
\begin{aligned}
\partial_{\text {cyc }}(1,2,3,4,5):=\{r(2,3,4,5)\}+ & \{r(3,4,5,1)\} \\
& +\{r(4,5,1,2)\}+\{r(5,1,2,3)\}+\{r(1,2,3,4)\} .
\end{aligned}
$$

We make a similar definition for the case of five intersecting lines. If the lines are (12), (13), (14), (15), (16), then we define

$$
\begin{aligned}
\partial_{\text {cyc }}(1 \mid 2,3,4,5,6):=\{r(1 \mid 2,3,4,5)\}+\{r(1 \mid 3,4,5,6)\} \\
+\{r(1 \mid 4,5,6,2)\}+\{r(1 \mid 5,6,2,3)\}+\{r(1 \mid 6,2,3,4)\} .
\end{aligned}
$$

Then,

$$
\begin{gathered}
\mathrm{B}_{2}^{(123)}=\partial_{\text {сус }}(-(1 \mid 2,3,4,34 \cap 56,5)+(3 \mid 12 \cap 56,1,2,4,6) \\
-(4 \mid 12 \cap 56,1,2,3,5)+(2 \mid 1,3,4,34 \cap 56,5)), \\
\mathrm{B}_{2}^{(124)}=\partial_{\text {сус }}((1,2,4,5,6)-(4 \mid 6,12 \cap 56,1,2,3)+(4 \mid 5,12 \cap 56,1,2,3)) .
\end{gathered}
$$

Notice that the configuration $(1,2,4,5,6)$ in $\mathbb{P}^{2}$ by duality, or by drawing the unique conic through these five points, determines the configuration of five points on $\mathbb{P}^{1}$.

Thus not only the functional equation (B.1) itself, but also the way it vanishes in $\mathrm{B}_{2} \otimes \mathbb{C}^{*}$ is of cluster origin: the five-term relations (B.10)-(B.11) correspond to certain pentagon faces in the four-dimensional Stasheff polytope of type $D_{4}$ describing the cluster $\mathcal{X}$-variety $\operatorname{Conf}_{6}\left(\mathbb{P}^{2}\right)$.

To complete the proof of the theorem it is sufficient to check that a certain specialization of the functional equation is zero. We leave this as an exercise.

Finally, one may look for functional equations for the trilogarithm which can be expressed via cluster $\mathcal{X}$-coordinates on the space $\operatorname{Conf}_{7}\left(\mathbb{P}^{2}\right)$, which is the case of interest for scattering amplitudes. The six-point identity in eq. (B.1) can obviously also be used for seven points in $\mathbb{P}^{2}$ : as written, it simply does not depend on the seventh point. Other 40-term identities can be obtained from the eq. (B.1) by applying parity conjugation and dihedral permutations of the points $1, \ldots, 7$. A less obvious type of transformation is parity 
followed by a transposition of two points. This results in transformations sending points in $\mathbb{P}^{2}$ to lines in $\mathbb{P}^{2}$. An example of such a transformation is

$$
1 \rightarrow(34), \quad 2 \rightarrow(35), \quad 3 \rightarrow(56), \quad 4 \rightarrow(67), \quad 5 \rightarrow(17), \quad 6 \rightarrow(12)
$$

Remarkably, applying this map to the 40-term identity (B.1) we get a functional equation written via cluster $\mathcal{X}$-coordinates on the space $\operatorname{Conf}_{7}\left(\mathbb{P}^{2}\right)$. After considering all possible transformations of these types we obtain a total of 35 different 40-term identities written via cluster $\mathcal{X}$-coordinates on the space $\operatorname{Conf}_{7}\left(\mathbb{P}^{2}\right)$, consisting of five families of seven related to each other by cyclic rotations of the seven points. Only 22 of these 35 identities are linearly independent. Of course all of them reduce to the identity in eq. (B.1) by some in general complicated change of variables. Such changes of variable arise from an embedding of the $D_{4}$ cluster algebra (that is, $\operatorname{Gr}(3,6)$ ) into the $E_{6}$ (or $\operatorname{Gr}(3,7)$ ) cluster algebra. It would be very interesting to search for new functional identities of cluster type at higher weight. Of particular interest is, of course, the next case - weight 4 . We have found that there are no identities at weight 4 involving cluster $\mathcal{X}$-coordinates on $\operatorname{Conf}_{8}\left(\mathbb{P}^{2}\right)$, but we do expect such identities on $\operatorname{Conf}_{8}\left(\mathbb{P}^{3}\right)$.

Open Access. This article is distributed under the terms of the Creative Commons Attribution License (CC-BY 4.0), which permits any use, distribution and reproduction in any medium, provided the original author(s) and source are credited.

\section{References}

[1] M.L. Mangano and S.J. Parke, Multiparton amplitudes in gauge theories, Phys. Rept. 200 (1991) 301 [hep-th/0509223] [INSPIRE].

[2] L.J. Dixon, Calculating scattering amplitudes efficiently, hep-ph/9601359 [INSPIRE].

[3] F. Cachazo and P. Svrček, Lectures on twistor strings and perturbative Yang-Mills theory, PoS (RTN2005) 004 [hep-th/0504194] [INSPIRE].

[4] Z. Bern, L.J. Dixon and D.A. Kosower, On-shell methods in perturbative QCD, Annals Phys. 322 (2007) 1587 [arXiv:0704.2798] [INSPIRE].

[5] R. Roiban, M. Spradlin and A. Volovich, Scattering amplitudes in gauge theories: Progress and outlook, J.Phys. A 44 (2011) 450301.

[6] B. Feng and M. Luo, An introduction to on-shell recursion relations, Front. Phys. ,2012,7 (5) :533-575 [arXiv: 1111.5759] [INSPIRE].

[7] L. Brink, J.H. Schwarz and J. Scherk, Supersymmetric Yang-Mills theories, Nucl. Phys. B 121 (1977) 77 [INSPIRE].

[8] F. Gliozzi, J. Scherk and D.I. Olive, Supersymmetry, supergravity theories and the dual spinor model, Nucl. Phys. B 122 (1977) 253 [INSPIRE].

[9] A. Goncharov, Galois symmetries of fundamental groupoids and noncommutative geometry, Duke Math. J. 128 (2005) 209 [math/0208144] [INSPIRE].

[10] O. Schlotterer and S. Stieberger, Motivic multiple Zeta values and superstring amplitudes, J. Phys. A 46 (2013) 475401 [arXiv:1205.1516] [InSPIRE]. 
[11] J. Drummond and É. Ragoucy, Superstring amplitudes and the associator, JHEP 08 (2013) 135 [arXiv: 1301.0794] [INSPIRE].

[12] J. Broedel, O. Schlotterer and S. Stieberger, Polylogarithms, multiple Zeta values and superstring amplitudes, Fortsch. Phys. 61 (2013) 812 [arXiv:1304.7267] [INSPIRE].

[13] J. Broedel, O. Schlotterer, S. Stieberger and T. Terasoma, All order $\alpha^{\prime}$-expansion of superstring trees from the Drinfeld associator, arXiv:1304.7304 [INSPIRE].

[14] J. Drummond, J. Henn, V. Smirnov and E. Sokatchev, Magic identities for conformal four-point integrals, JHEP 01 (2007) 064 [hep-th/0607160] [INSPIRE].

[15] Z. Bern, M. Czakon, L.J. Dixon, D.A. Kosower and V.A. Smirnov, The four-loop planar amplitude and cusp anomalous dimension in maximally supersymmetric Yang-Mills theory, Phys. Rev. D 75 (2007) 085010 [hep-th/0610248] [INSPIRE].

[16] L.F. Alday and J.M. Maldacena, Gluon scattering amplitudes at strong coupling, JHEP 06 (2007) 064 [arXiv:0705.0303] [INSPIRE].

[17] J. Drummond, G. Korchemsky and E. Sokatchev, Conformal properties of four-gluon planar amplitudes and Wilson loops, Nucl. Phys. B 795 (2008) 385 [arXiv:0707.0243] [INSPIRE].

[18] J. Drummond, J. Henn, G. Korchemsky and E. Sokatchev, On planar gluon amplitudes/Wilson loops duality, Nucl. Phys. B 795 (2008) 52 [arXiv:0709.2368] [INSPIRE].

[19] L.F. Alday and J. Maldacena, Comments on gluon scattering amplitudes via AdS/CFT, JHEP 11 (2007) 068 [arXiv:0710.1060] [INSPIRE].

[20] J. Drummond, J. Henn, G. Korchemsky and E. Sokatchev, Conformal Ward identities for Wilson loops and a test of the duality with gluon amplitudes, Nucl. Phys. B 826 (2010) 337 [arXiv:0712.1223] [INSPIRE].

[21] J. Drummond, J. Henn, G. Korchemsky and E. Sokatchev, Dual superconformal symmetry of scattering amplitudes in $N=4$ super-Yang-Mills theory, Nucl. Phys. B 828 (2010) 317 [arXiv: 0807.1095] [INSPIRE].

[22] A.B. Goncharov, M. Spradlin, C. Vergu and A. Volovich, Classical polylogarithms for amplitudes and Wilson loops, Phys. Rev. Lett. 105 (2010) 151605 [arXiv:1006.5703] [INSPIRE].

[23] A. Goncharov, Geometry of configurations, polylogarithms, and motivic cohomology, Adv. Math. 114 (1995) 197.

[24] V. Fock and A. Goncharov, Cluster ensembles, quantization and the dilogarithm, Ann. Sci. Éc. Norm. Supér. 42 (2009) 865 [math/0311245] [INSPIRE].

[25] S. Fomin and A. Zelevinsky, Cluster algebras. I: foundations, J. Am. Math. Soc. 15 (2002) 497.

[26] S. Fomin and A. Zelevinsky, Cluster algebras. II: finite type classification, Invent. Math. $\mathbf{1 5 4}$ (2003) 63.

[27] N. Arkani-Hamed, F. Cachazo, C. Cheung and J. Kaplan, A duality for the S matrix, JHEP 03 (2010) 020 [arXiv: 0907.5418] [INSPIRE].

[28] N. Arkani-Hamed, F. Cachazo and C. Cheung, The grassmannian origin of dual superconformal invariance, JHEP 03 (2010) 036 [arXiv:0909.0483] [INSPIRE].

[29] N. Arkani-Hamed, J. Bourjaily, F. Cachazo and J. Trnka, Local spacetime physics from the grassmannian, JHEP 01 (2011) 108 [arXiv:0912.3249] [INSPIRE]. 
[30] N. Arkani-Hamed, J. Bourjaily, F. Cachazo and J. Trnka, Unification of residues and grassmannian dualities, JHEP 01 (2011) 049 [arXiv:0912.4912] [INSPIRE].

[31] N. Arkani-Hamed, J.L. Bourjaily, F. Cachazo, S. Caron-Huot and J. Trnka, The all-loop integrand for scattering amplitudes in planar $N=4$ SYM, JHEP 01 (2011) 041 [arXiv: 1008.2958] [INSPIRE].

[32] N. Arkani-Hamed et al., Scattering amplitudes and the positive grassmannian, arXiv: 1212.5605 [INSPIRE].

[33] S. Fomin and A. Zelevinsky, $Y$-systems and generalized associahedra, Ann. Math. 158 (2003) 977.

[34] J.D. Stasheff, Homotopy associativity of H-spaces. I, Trans. Am. Math. Soc. 108 (1963) 275.

[35] J.D. Stasheff, Homotopy associativity of H-spaces. II, Trans. Am. Math. Soc. 108 (1963) 293.

[36] A. Hodges, Eliminating spurious poles from gauge-theoretic amplitudes, JHEP 05 (2013) 135 [arXiv: 0905.1473] [INSPIRE].

[37] R. Penrose, Twistor algebra, J. Math. Phys. 8 (1967) 345 [INSPIRE].

[38] R. Penrose and M.A. MacCallum, Twistor theory: an approach to the quantization of fields and space-time, Phys. Rept. 6 (1972) 241 [INSPIRE].

[39] E. Witten, Perturbative gauge theory as a string theory in twistor space, Commun. Math. Phys. 252 (2004) 189 [hep-th/0312171] [INSPIRE].

[40] L. Mason and D. Skinner, Dual superconformal invariance, momentum twistors and grassmannians, JHEP 11 (2009) 045 [arXiv:0909.0250] [INSPIRE].

[41] E. Witten, An interpretation of classical Yang-Mills theory, Phys. Lett. B 77 (1978) 394 [INSPIRE].

[42] N. Beisert and C. Vergu, On the geometry of null polygons in full $N=4$ superspace, Phys. Rev. D 86 (2012) 026006 [arXiv:1203.0525] [INSPIRE].

[43] N. Beisert, S. He, B.U. Schwab and C. Vergu, Null polygonal Wilson loops in full $N=4$ superspace, J. Phys. A 45 (2012) 265402 [arXiv:1203.1443] [INSPIRE].

[44] Z. Bern, L. Dixon, D. Kosower, R. Roiban, M. Spradlin et al., The two-loop six-gluon MHV amplitude in maximally supersymmetric Yang-Mills theory, Phys. Rev. D 78 (2008) 045007 [arXiv: 0803.1465] [INSPIRE].

[45] J. Drummond, J. Henn, G. Korchemsky and E. Sokatchev, Hexagon Wilson loop = six-gluon MHV amplitude, Nucl. Phys. B 815 (2009) 142 [arXiv:0803.1466] [INSPIRE].

[46] V. Del Duca, C. Duhr and V.A. Smirnov, An analytic result for the two-loop hexagon Wilson loop in $N=4$ SYM, JHEP 03 (2010) 099 [arXiv:0911.5332] [INSPIRE].

[47] V. Del Duca, C. Duhr and V.A. Smirnov, The two-loop hexagon Wilson loop in $N=4 S Y M$, JHEP 05 (2010) 084 [arXiv: 1003.1702] [INSPIRE].

[48] A. Beilinson, Height pairing between algebraic cycles, in K-theory, arithmetic and geometry, Y.I. Manin ed., Springer, Berlin Germany (1987).

[49] P. Deligne and A.B. Goncharov, Groupes fondamentaux motiviques de Tate mixte, Ann. Sci. École Norm. Sup. 38 (2005) 1.

[50] S.J. Bloch, Higher regulators, algebraic K-theory, and zeta functions of elliptic curves, American Mathematical Society, Providence U.S.A. (2000). 
[51] A. Suslin, $K_{3}$ of a field and the Bloch group, Proc. Steklov Inst. Math. 183 (1990) 217.

[52] D. Zagier, Polylogarithms, Dedekind zeta functions, and the algebraic K-theory of fields, in Arithmetic algebraic geometry, J.L. Colliot-Thelene et al., Boston U.S.A. (1991).

[53] A.B. Goncharov, Polylogarithms and motivic Galois groups, in Motives (Seattle, WA, 1991), American Mathematical Sociesty, Providence U.S.A. (1994).

[54] A. Goncharov, Deninger's conjecture on L-functions of elliptic curves at $s=3, J$. Math. Sci., New York 81 (1996) 2631 [alg-geom/9512016].

[55] S. Caron-Huot, Superconformal symmetry and two-loop amplitudes in planar $N=4$ super Yang-Mills, JHEP 12 (2011) 066 [arXiv:1105.5606] [INSPIRE].

[56] S. Fomin and A. Zelevinsky, The Laurent phenomenon, Adv. Appl. Math. 28 (2002) 119.

[57] J.S. Scott, Grassmannians and cluster algebras, Proc. Lond. Math. Soc. III Ser. 92 (2006) 345 .

[58] M. Gekhtman, M. Shapiro, and A. Vainshtein, Cluster algebras and Poisson geometry, Mosc. Math. J. 3 (2003) 899.

[59] B. Keller, Cluster algebras, quiver representations and triangulated categories, in Triangulated categories, Cambridge University Press, Cambridge U.K. (2010).

[60] S. Fomin and N. Reading, Root systems and generalized associahedra, in Geometric combinatorics, American Mathematical Society, Providence U.S.A. (2007).

[61] C. Anastasiou et al., Two-loop polygon Wilson loops in N=4 SYM, JHEP 05 (2009) 115 [arXiv: 0902.2245] [INSPIRE].

[62] L.J. Dixon, J.M. Drummond and J.M. Henn, Analytic result for the two-loop six-point NMHV amplitude in $N=4$ super Yang-Mills theory, JHEP 01 (2012) 024 [arXiv:1111.1704] [INSPIRE].

[63] L.J. Dixon, J.M. Drummond and J.M. Henn, Bootstrapping the three-loop hexagon, JHEP 11 (2011) 023 [arXiv:1108.4461] [INSPIRE].

[64] S. Caron-Huot and S. He, Jumpstarting the all-loop S-matrix of planar $N=4$ super Yang-Mills, JHEP 07 (2012) 174 [arXiv:1112.1060] [INSPIRE].

[65] V. Del Duca, C. Duhr and V.A. Smirnov, A two-loop octagon Wilson loop in $N=4 S Y M$, JHEP 09 (2010) 015 [arXiv: 1006.4127] [INSPIRE].

[66] P. Heslop and V.V. Khoze, Analytic results for MHV Wilson loops, JHEP 11 (2010) 035 [arXiv: 1007.1805] [INSPIRE].

[67] P. Heslop and V.V. Khoze, Wilson loops 3-loops in special kinematics, JHEP 11 (2011) 152 [arXiv: 1109.0058] [INSPIRE].

[68] T. Goddard, P. Heslop and V.V. Khoze, Uplifting amplitudes in special kinematics, JHEP 10 (2012) 041 [arXiv:1205.3448] [INSPIRE].

[69] L. Ferro, Differential equations for multi-loop integrals and two-dimensional kinematics, JHEP 04 (2013) 160 [arXiv: 1204.1031] [INSPIRE].

[70] J. Bartels, L. Lipatov and A. Prygarin, Collinear and Regge behavior of $2 \rightarrow 4 M H V$ amplitude in $N=4$ super Yang-Mills theory, arXiv:1104.4709 [INSPIRE]. 
[71] A. Prygarin, M. Spradlin, C. Vergu and A. Volovich, All two-loop MHV amplitudes in multi-Regge kinematics from applied symbology, Phys. Rev. D 85 (2012) 085019 [arXiv:1112.6365] [INSPIRE].

[72] J. Bartels, A. Kormilitzin, L. Lipatov and A. Prygarin, BFKL approach and $2 \rightarrow 5$ maximally helicity violating amplitude in $\mathcal{N}=4$ super-Yang-Mills theory, Phys. Rev. D 86 (2012) 065026 [arXiv:1112.6366] [INSPIRE].

[73] L. Lipatov, A. Prygarin and H.J. Schnitzer, The multi-Regge limit of NMHV amplitudes in $N=4 S Y M$ theory, JHEP 01 (2013) 068 [arXiv:1205.0186] [INSPIRE].

[74] L.J. Dixon, C. Duhr and J. Pennington, Single-valued harmonic polylogarithms and the multi-Regge limit, JHEP 10 (2012) 074 [arXiv:1207.0186] [INSPIRE].

[75] J. Pennington, The six-point remainder function to all loop orders in the multi-Regge limit, JHEP 01 (2013) 059 [arXiv:1209.5357] [INSPIRE].

[76] L.F. Alday, D. Gaiotto, J. Maldacena, A. Sever and P. Vieira, An operator product expansion for polygonal null Wilson loops, JHEP 04 (2011) 088 [arXiv: 1006.2788] [INSPIRE].

[77] D. Gaiotto, J. Maldacena, A. Sever and P. Vieira, Bootstrapping null polygon Wilson loops, JHEP 03 (2011) 092 [arXiv: 1010.5009] [INSPIRE].

[78] D. Gaiotto, J. Maldacena, A. Sever and P. Vieira, Pulling the straps of polygons, JHEP 12 (2011) 011 [arXiv:1102.0062] [INSPIRE].

[79] B. Basso, A. Sever and P. Vieira, Space-time S-matrix and flux-tube S-matrix at finite coupling, Phys. Rev. Lett. 111 (2013) 091602 [arXiv:1303.1396] [INSPIRE].

[80] N. Beisert et al., Review of AdS/CFT integrability: an overview, Lett. Math. Phys. 99 (2012) 3 [arXiv: 1012.3982] [INSPIRE].

[81] J.M. Drummond, J.M. Henn and J. Plefka, Yangian symmetry of scattering amplitudes in $N=4$ super Yang-Mills theory, JHEP 05 (2009) 046 [arXiv:0902.2987] [INSPIRE].

[82] L.F. Alday, D. Gaiotto and J. Maldacena, Thermodynamic bubble ansatz, JHEP 09 (2011) 032 [arXiv: 0911.4708] [INSPIRE].

[83] L.F. Alday, J. Maldacena, A. Sever and P. Vieira, Y-system for scattering amplitudes, J. Phys. A 43 (2010) 485401 [arXiv: 1002.2459] [InSPIRE].

[84] L. Ferro, T. Lukowski, C. Meneghelli, J. Plefka and M. Staudacher, Harmonic R-matrices for scattering amplitudes and spectral regularization, Phys. Rev. Lett. 110 (2013) 121602 [arXiv:1212.0850] [INSPIRE].

[85] V. Del Duca et al., The one-loop six-dimensional hexagon integral with three massive corners, Phys. Rev. D 84 (2011) 045017 [arXiv:1105.2011] [InSPIRE].

[86] C. Duhr, H. Gangl and J.R. Rhodes, From polygons and symbols to polylogarithmic functions, JHEP 10 (2012) 075 [arXiv: 1110.0458] [INSPIRE].

[87] M. Bullimore and D. Skinner, Descent equations for superamplitudes, arXiv:1112.1056 [INSPIRE].

[88] A. Brandhuber, G. Travaglini and G. Yang, Analytic two-loop form factors in $N=4 S Y M$, JHEP 05 (2012) 082 [arXiv: 1201.4170] [InSPIRE].

[89] C. Bogner and F. Brown, Symbolic integration and multiple polylogarithms, PoS(LL2012) 053 [arXiv: 1209.6524] [INSPIRE]. 
[90] A.E. Lipstein and L. Mason, From the holomorphic Wilson loop to 'd log' loop-integrands for super-Yang-Mills amplitudes, JHEP 05 (2013) 106 [arXiv:1212.6228] [INSPIRE].

[91] S.G. Naculich, H. Nastase and H.J. Schnitzer, All-loop infrared-divergent behavior of most-subleading-color gauge-theory amplitudes, JHEP 04 (2013) 114 [arXiv:1301.2234] [INSPIRE].

[92] J. Drummond et al., Leading singularities and off-shell conformal integrals, JHEP 08 (2013) 133 [arXiv: 1303.6909] [INSPIRE].

[93] C. Duhr, Hopf algebras, coproducts and symbols: an application to Higgs boson amplitudes, JHEP 08 (2012) 043 [arXiv: 1203.0454] [inSPIRE].

[94] A. von Manteuffel and C. Studerus, Top quark pairs at two loops and Reduze 2, PoS (LL2012) 059 [arXiv: 1210.1436] [INSPIRE].

[95] T. Gehrmann, L. Tancredi and E. Weihs, Two-loop QCD helicity amplitudes for $g g \rightarrow Z g$ and $g g \rightarrow Z \gamma$, JHEP 04 (2013) 101 [arXiv:1302.2630] [INSPIRE].

[96] C. Anastasiou, C. Duhr, F. Dulat and B. Mistlberger, Soft triple-real radiation for Higgs production at N3LO, JHEP 07 (2013) 003 [arXiv: 1302.4379] [INSPIRE].

[97] J.M. Henn, Multiloop integrals in dimensional regularization made simple, Phys. Rev. Lett. 110 (2013) 251601 [arXiv:1304.1806] [INSPIRE].

[98] C.F. Gauss, Pentagramma mirificum, in Werke, Band III, Göttingen Germany (1863). 